Journal of Statistical Physics, Vol. 23, No. 3, 1980

\title{
Kinetic Theory of Hydrodynamic Flows. I. The Generalized Normal Solution Method and Its Application to the Drag on a Sphere
}

\author{
Henk van Beijeren ${ }^{1}$ and J. R. Dorfman ${ }^{2}$
}

\begin{abstract}
Received December 14, 1979
We consider the flow of a dilute gas around a macroscopic heavy object. The state of the gas is described by an extended Boltzmann equation where the interactions between the gas molecules and the object are taken into account in computing the rate of change of the distribution function of the gas. We then show that the extended Boltzmann is equivalent to the usual Boltzmann equation, supplemented by boundary conditions imposed on the distribution function at the surface of the object. The remainder of the paper is devoted to a study of the solution of the extended Boltzmann equation in the case that the mean free path of a gas molecule is small compared to some characteristic dimension of the macroscopic object. We show that the Chapman-Enskog normal solution of the ordinary Boltzmann equation is not in general a solution of the extended equation near the surface of the object and must be supplemented by a boundary layer term. We then introduce a projection operator method which allows us to decompose the solution of the extended equation into a normal solution part and a boundary layer part when the gas flow is sufficiently slow. As a specific example of the method we consider the flow around a sphere, and derive the Stokes-Boussinesq form for the frequency-dependent force on the sphere for arbitrary slip coefficient. This derivation is the first one that starts from the Boltzmann equation for a general dilute gas and incorporates the effect of the boundary layer on the drag force.
\end{abstract}

KEY WORDS: Boltzmann equation; boundary conditions; normal solutions; projection operator; Stokes' law; boundary layer; slip coefficients; drag force.

Work supported by the National Science Foundation.

${ }^{1}$ Institut für Theoretische Physik, Rhein-Westf. Technische Hochschule, Aachen, West Germany.

${ }^{2}$ Institute for Physical Science and Technology and Department of Physics and Astronomy, University of Maryland, College Park, Maryland. 


\section{INTRODUCTION}

In a series of four papers, of which this is the first, we use the kinetic theory of gases to study the nonequilibrium properties of a dilute gas when there is a large object moving in it. The first two papers will be devoted to an analysis of the force on a large sphere or cylinder moving in the gas, the third will treat the torque on a sphere rotating in the gas, and the fourth paper will treat the diffusion of a large particle undergoing Brownian motion in the gas.

In the first two papers, our treatment of the force on the object in the gas will be based on the Boltzmann equation, or rather an extension of it designed to take into account the effect of the molecule-object collisions on the distribution function for the gas. ${ }^{(1,2)}$ At first glance it may seem somewhat superfluous to spend any effort on a new derivation of the force exerted by a gas on a sphere or cylinder from the Boltzmann equation under conditions where one might expect continuum hydrodynamics to apply. One can argue that the Chapman-Enskog normal solution of the Boltzmann equation $^{(3)}$ leads directly to the Navier-Stokes hydrodynamic equations, from which the Stokes and Boussinesq forms for the drag force on a sphere can be derived. However, this argument does not take into account the fact that there is an important restriction on the Chapman-Enskog solution, namely, it is implicitly assumed that there are no boundaries or large objects present in the system. If, however, there are objects or boundaries, it is well known that the Chapman-Enskog solution breaks down near them. ${ }^{(6,7)}$ This breakdown is associated with the existence of a kinetic boundary layer next to the boundary which is on the order of a mean free path thick and where there is a disturbance of the normal solution caused by collisions of the gas particles with the boundary. Furthermore, a complete determination of the normal solution far from the boundaries requires that boundary conditions on the hydrodynamic functions be obtained from the microscopic mechanism for reflection of the gas particles at the boundary, and such boundary conditions are not determined by the Chapman-Enskog solution.

In view of these comments, it would seem appropriate to present a derivation of the hydrodynamic results for the force on an object using a kinetic theory where the presence of boundaries or objects is taken into account from the very beginning, and where the effects of the kinetic boundary layer can be estimated. Such a program for the derivation of hydrodynamic results from the Boltzmann equation is not new, and a number of cases have already been treated. ${ }^{(6,7)}$ What is new in our work is the attempt to base a kinetic theory of hydrodynamic flows on the extended Boltzmann equation, which will be described in the next section. We will show in this paper and in those following it that the use of the extended Boltzmann equation has the following advantages: 
(a) We will be able to express the effects of boundaries or of objects in the gas on the distribution function in terms of some compact, formal expressions. Although these expressions are difficult to evaluate in general, they will be quite useful in our analysis, and represent a technical advance over the earlier and more cumbersome methods of analyzing boundary effects.

(b) We will be able to clarify the dynamical origin of hydrodynamic results, such as Stokes' law for the force on a sphere, in terms of collision processes taking place in the gas.

By analyzing hydrodynamic results such as Stokes' law in terms of microscopic collision processes, we will be able to make some progress toward an understanding of why hydrodynamic concepts and results are often very useful in describing processes of diffusion or Brownian motion in fluids, even when the diffusing particle is identical to the other particles in the fluid (see Ref. 8 for a review).

Now we turn our attention to the main subject of the present paper, the calculation of the drag force on a large sphere or cylinder moving in a dilute gas. In general the drag force is a function of several parameters. Let $V$ be a characteristic velocity of the gas stream around the object; $c$ the velocity of sound in the gas; $R$ a characteristic size of the object; $l$ the mean free path of a molecule in the gas; $a$ the range of the intermolecular force; $M$ the mass of the object; and $m$ the mass of a gas molecule. From these one can construct the following dimensionless quantities: the Mach number $\mathscr{H}=$ $V / c$, the Knudsen number $\mathscr{K}=l / R$, the ratio $a / l$ of the intermolecular force range to the mean free path, and the mass ratio $m / M$. The properties of the gas flow depend on all these parameters, and the determination of this dependence is one of the central problems of kinetic theory.

In our treatment we restrict ourselves to the case where three of these parameters plus a product of two of them, namely $\mathscr{M}, a / l, m / M$, and $a / R$, are all much less than unity, that is, we discuss the flow of a dilute gas around a macroscopic sphere or cylinder at small Mach numbers only.

So far most of the work on these flows has been concentrated on the limiting cases where $\mathscr{K} \ll 1$, the Stokes or Clausius regime, where the mean free path is much smaller than the characteristic size of the object, and $\mathscr{K} \gg 1$, the rarefied or Knudsen regime. ${ }^{(6,7)}$

For $\mathscr{K} \ll 1$ the Boltzmann equation, together with the microscopic reflection law for gas molecules hitting the surface of the object, should give the Stokes-Boussinesq results for the force on a sphere, say. More generally, it should produce the complete distribution function outside the sphere; that is, it should not only give the normal distribution function, which prevails outside the kinetic boundary layer, and which is completely determined by the boundary conditions for the hydrodynamic densities (number, momentum, 
and energy density) at the surface of the sphere, but it also ought to yield the kinetic boundary layer which is present close to the sphere.

There has been a considerable amount of work on the derivation of continuum hydrodynamics from the Boltzmann equation as well as on the determination of the properties of the kinetic boundary layer and of the boundary conditions satisfied by the hydrodynamic densities. ${ }^{(2,6,7)}$ Here we mention some of the main results. The hydrodynamic boundary conditions have been obtained usually by considering flow patterns with onedimensional geometry bounded by a semiinfinite flat plate, since locally this should be a good approximation for any macroscopic, smooth surface. Maxwell was the first to obtain qualitatively correct boundary conditions for this case. ${ }^{(9)}$. Most models that have been studied so far for the reflection of gas particles at the surface give rise to the so-called stick boundary condition, where the average velocity and the temperature of the gas at the boundary surface coincide with the velocity and temperature of the surface. In the special case of specular reflections, however, one obtains slip boundary conditions; only the velocity components normal to the surface coincide for both gas and object. In addition, both the tangential stress exerted by the gas on the boundary and the temperature gradient normal to the surface vanish. In general, there are corrections to these boundary conditions which are proportional to the Knudsen number $\mathscr{K}$.

These corrections involve a new set of coefficients, called slip coefficients, ${ }^{3}$ whose values depend on the structure of the boundary layer. Although there are a few examples where the simple one-dimensional geometry and a simplified gas model allow an exact calculation of boundary layer effects, in general slip coefficients cannot be calculated exactly. Instead one must resort to variational methods or moment expansions to calculate them. Recently, Scharf ${ }^{(10)}$ and later McLennan and $\mathrm{Chiu}^{(11)}$ presented a derivation of Stokes' law together with slip coefficients from the Boltzmann equation to which they added source terms to represent the interaction of the gas molecules with the moving sphere. However, these source terms were not derived from a microscopic molecule-sphere interaction model. Later we will comment in more detail on the precise connection between their work and that presented in this paper.

It is also worth mentioning here the main features of the flows that obtain when the Knudsen number is not small, since we will have occasion to refer to these cases also. For $\mathscr{K} \gg 1$, the case of rarefied gas flows, the Boltzmann equation is usually solved by an iterative method. ${ }^{(1,2,12)}$ The most straightforward iteration gives rise to an expansion of the drag force in powers of the density of the gas. The coefficients in this expansion,

\footnotetext{
${ }^{3}$ See Ref. 5 for a discussion of the slip coefficient in hydrodynamics.
} 
however, are divergent except for the first few. The reasons for this divergence are the same as those for the divergences in the density expansion of transport coefficients like shear viscosity and heat conductivity. As in those cases, the divergences can be removed by a partial resummation of diverging terms, and, as in those cases, nonanalytic terms in the density expansion appear. For certain models a few terms are known in this resummed expansion. ${ }^{(12,13)}$

For the intermediate region where $\mathscr{K}$ is neither very large nor very small few results are known. Cercignani and co-workers have employed variational methods to calculate in the BGK model the general $\mathscr{K}$ dependence of several quantities, among which is the drag force on a sphere. ${ }^{(6,14)}$ The agreement of their results with experiments on real gases is remarkably good.

The subject of this paper is a calculation of the drag force on a sphere in the hydrodynamic regime by an extension of the Chapman-Enskog normal solution method, where the existence and properties of the kinetic boundary layer are taken explicitly into account. To do this the interactions between the gas molecules and the sphere can be treated as an additional term in the Boltzmann equation describing the rate of change of the distribution function due to such interactions. Since this term is localized on the surface of the sphere, it can be treated as a source term for the ordinary Boltzmann equation. It is a source term which does depend on the form of the distribution function at the surface, but given this, the source term is determined entirely by the gas-surface interaction mechanism. The solution of the Boltzmann equation extended with these extra terms is obtained by expanding the distribution function about total equilibrium and by projecting it onto the space of normal solutions of the ordinary Boltzmann equation. Because the kinetic boundary layer cannot be calculated in practice, the solution of the extended Boltzmann equation is obtained to leading order in $\mathscr{K}$ only. For different reflection mechanisms the expected boundary conditions for the fluid fields are obtained and Stokes' law for the force on a sphere is recovered.

In a second paper we treat the solution of the linearized extended Boltzmann equation by means of an iterative expansion. The iterates are interpreted straightforwardly to describe certain well-defined dynamical events in the system. In the rarefied gas case identification with the usual expansions is direct and also the usual resummations can be interpreted simply in terms of dynamical events. In the hydrodynamic regime the dynamical processes are dominated by propagation through hydrodynamic modes and reflections at the boundary of the sphere. Therefore the time evolution operator for the distribution function is separated into a hydrodynamic and an orthogonal part with the aid of the same projection operator that was used in the first paper. The iterative expansion is then regrouped into terms containing one, two, three, etc., hydrodynamic propagators. The con- 
tributions of these terms to the distribution function form a series whose formal summation leads to precisely the same equations and results as obtained in the first paper.

Since both the rarefied and hydrodynamic gas flows can be described by the same extended Boltzmann equation, it follows that the same dynamical events are responsible for the shape of the distribution function and for the drag force in the hydrodynamic regime as in the rarefied gas regime. The difference is, of course, that the contributions from various dynamical events differ in the various regimes. However, in neither the rarefied regime nor the hydrodynamic regime is the most straightforward iteration of the Boltzmann equation convergent, which means that the behavior of the distribution function is always dominated by collective effects. In the rarefied gas case this collective effect is the damping of free particle propagation by random collisions with other gas molecules. In the hydrodynamic regime the same effect is present, but it is dominated in turn by the collective action of the hydrodynamic modes excited by repeated collisions of the gas molecules with the sphere.

An extra complication occurs in the case of the drag on an infinitely long cylinder. In this case the stationary linearized hydrodynamic equations do not even have a solution, which can be traced back to the circumstance that the influence of the average motion of the fluid far from the cylinder may not be neglected. ${ }^{(4)}$ If one includes this effect by linearizing about the equilibrium distribution function valid far from the cylinder, and not about one in the rest frame of the cylinder, no problems arise and both the generalized Chapman-Enskog method and the iterative method can be applied as before. In the hydrodynamic equations this leads to the well known Oseen term. ${ }^{(15)}$ In our third paper the same iterative method is employed to calculate the torsional drag on a rotating sphere or cylinder.

Finally, the iterative method is also of interest because it provides a link between the theory of Brownian motion and the theory of diffusion of a microscopic particle. This is treated in our fourth paper.

The organization of this paper is as follows: In Section 2 we introduce the extended Boltzmann equation for a dilute gas in contact with a sphere. In Section 3 we discuss the Chapman-Enskog normal solution and its shortcomings if a boundary is present. In Section 4 the extended Boltzmann equation is linearized and the distribution function is separated, by a projection onto hydrodynamic modes, into a normal part and a boundary layer part. In Section 5 the projection operator used is critically examined and found to possess an unphysical feature-namely that after the application of the projection operator the source term in the equation for the projected part of the distribution function is not confined to the surface of the sphere, but rather the source becomes spread out over a distance of a few 
mean free paths around the sphere. This is repaired by the introduction of a slightly different projection operator that keeps the sources confined to the surface of the sphere. In Section 6 the extended Boltzmann equation is solved to leading order in the Knudsen number for a few different reflection laws at the surface of the sphere. The normal part of the resulting distribution function and the drag on the sphere are calculated. The main computational result of this paper is given by $\mathrm{Eq}$. (6.43), where we obtain the frequency-dependent form of Stokes' law appropriate for the various kinds of molecule-sphere collision mechanisms considered here. In Section 7 we consider an alternative approach to this solution of the difficulties discussed in Section 5 by passing directly to the continuum limit, $\mathscr{K} \rightarrow 0$. The boundary layer effects can still be taken into account in the limit, and we again recover Stokes' law.

\section{THE EXTENDED BOLTZMANN EQUATION}

We consider the equation satisfied by $f(\mathbf{r}, \mathbf{v}, t)$, the single-particle distribution function of the gas. We take $f(\mathbf{r}, \mathbf{v}, t)$ to be normalized so that $f(\mathbf{r}, \mathbf{v}, t) d \mathbf{r} d \mathbf{v}$ is the number of gas particles in $d \mathbf{r}$ about $\mathbf{r}$ and in $d \mathbf{v}$ about $v$ at time $t$. In the absence of external forces, but in the presence of a macroscopic object, $f(\mathbf{r}, \mathbf{v}, t)$ can change in time through three different processes:

(i) Free streaming, where molecules at $(\mathbf{r}, \mathbf{v})$ at time $t$ move to $(\mathbf{r}+\mathbf{v} d t, \mathbf{v})$ at time $t+d t$.

(ii) Collisions of gas molecules with each other. The effects of these collisions on $f(\mathbf{r}, \mathbf{v}, t)$ is taken to be given by the usual Boltzmann collision operator.

(iii) Collisions of the gas molecules with the object. These collisions also cause $f(\mathbf{r}, \mathbf{v}, t)$ to change in time. Although they are usually treated by formulating their effect on $f$ as a boundary condition, there is no need to do so, and the result of these collisions can be incorporated directly into the Boltzmann equation.

Furthermore, the macroscopic object will be accelerated by its collisions with gas molecules and possibly also by some external force. It is more convenient then to use an (accelerated) coordinate system in which the object is at rest at all times. In this coordinate frame virtual forces acting on the particles are an additional source of change for the distribution function. A complication is that the actual acceleration of the object due to collisions with gas molecules is rapidly fluctuating in time, and also it contains angular as well as linear components. These fluctuations, however, are a result of fluctuations in the one-particle distribution function of the gas molecules and it is not our intention here to include these in the description 
of the physical system under consideration. Instead, we will only take into account the average acceleration of the object by the gas molecules, as would follow from a smoothed-out distribution function, such as the one occurring in the Boltzmann equation. If one realizes that the fluctuations in the velocity of the object are on the order of the thermal velocity $\left(k_{B} T / M\right)^{1 / 2}$, it becomes clear that this approximation must be very good as long as the object has a velocity of macroscopic size. Furthermore, if, for instance, the system (including the flow pattern) has rotational symmetry about some axis, the average angular acceleration vanishes.

Taking into account all four above-mentioned processes by which $f(\mathbf{r}, \mathbf{v}, t)$ may change in time, we are led to the extended Boltzmann equation

$$
\frac{\partial f(\mathbf{r}, \mathbf{v}, t)}{\partial t}=-\mathbf{v} \cdot \nabla f(\mathbf{r}, \mathbf{v}, t)+\mathbf{a}(t) \cdot \frac{\partial}{\partial \mathbf{v}} f(\mathbf{r}, \mathbf{v}, t)+J(f, f)+\overline{\mathbf{T}} f(\mathbf{r}, \mathbf{v}, t)
$$

The term $-\mathbf{v} \cdot \nabla f$ accounts for the change in $f$ due to free streaming and $\mathbf{a} \cdot(\partial / \partial \mathbf{v}) f$ describes the change in $f$ due to the virtual forces acting on the gas particles in the accelerated reference frame. The quantity a is related to the (average) velocity $\mathbf{V}$ of the object by the relation $\mathbf{a}(t)=+d \mathbf{V}(t) / d t$. The term $J(f, f)$ takes into account the intermolecular collisions and is given by ${ }^{(2,3,6,7)}$

$$
\begin{aligned}
J(f, f)= & \int d \mathbf{v}_{1} \int_{0}^{a} b d b \int_{0}^{2 \pi} d \phi\left|\mathbf{v}-\mathbf{v}_{1}\right| \\
& \times\left[f\left(\mathbf{r}, \mathbf{v}^{\prime}, t\right) f\left(\mathbf{r}, \mathbf{v}_{1}{ }^{\prime}, t\right)-f(\mathbf{r}, \mathbf{v}, t) f\left(\mathbf{r}, \mathbf{v}_{1}, t\right)\right]
\end{aligned}
$$

where $b$ and $\phi$ are respectively the impact parameter and the azimuthal angle describing a two-body collision, $a$ is the range of the intermolecular potential, which may be infinite, and $\mathbf{v}^{\prime}$ and $\mathbf{v}_{1}{ }^{\prime}$ are the restituting velocities. The term $\overline{\mathbf{T}} f$ describes the rate of change of $f$ due to molecule-object collisions. The precise form of $\overline{\mathbf{T}}$ depends on the shape of the object and the interaction mechanism between the molecules and the object. ${ }^{(2)}$ In the case that the object is a sphere, a fairly general form of $\overline{\mathbf{T}}$ is given by

$$
\begin{aligned}
\overline{\mathbf{T}} f(\mathbf{r}, \mathbf{v})= & R^{2} \int d \hat{\boldsymbol{\sigma}} \delta(\mathbf{r}-R \hat{\boldsymbol{\sigma}})\left[\int d \mathbf{v}^{\prime} P\left(\mathbf{v}^{\prime}, \mathbf{v}, \hat{\boldsymbol{\sigma}}\right)\left|\mathbf{v}^{\prime} \cdot \hat{\boldsymbol{\sigma}}\right| f^{+}\left(\mathbf{r}, \mathbf{v}^{\prime}\right)\right. \\
& \left.-\Theta(-\mathbf{v} \cdot \hat{\boldsymbol{\sigma}})|\mathbf{v} \cdot \hat{\boldsymbol{\sigma}}| f^{+}(\mathbf{r}, \mathbf{v})\right]
\end{aligned}
$$

Here $R$ is the radius of the sphere, which is assumed to be centered at the origin, $\hat{\boldsymbol{\sigma}}$ denotes a unit vector, the $\hat{\boldsymbol{\sigma}}$-integration runs over the unit sphere, and $\Theta(x)$ is the unit step function $[\Theta(x)=1$ for $x \geqslant 0$ and vanishes elsewhere]. By $f^{+}$we mean the limit for $f$ as the surface of the sphere is approached from the outside. The action of the operator $\overline{\mathbf{T}}$ can be understood with the aid of Fig. 1. We take the vertical axis to be in the direction of 


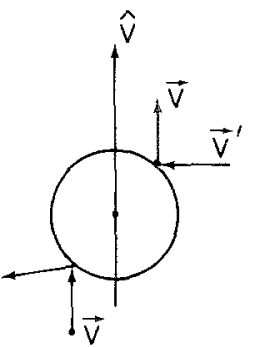

(a)

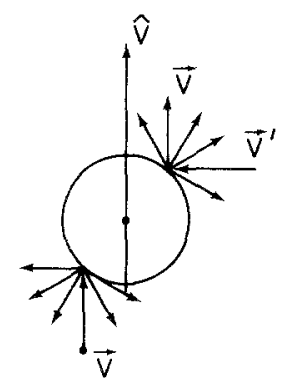

(b)

Fig. 1

$\mathbf{v}$. To compute the rate of decrease of particles with velocity $\mathbf{v}$ through collisions with the sphere, we note that such particles will collide with the sphere at a point $\mathbf{r}=R \hat{\sigma}$ on the hemisphere $\mathbf{v} \cdot \hat{\boldsymbol{\sigma}}<0$. These collisions are accounted for by the second term on the right-hand side of (2.3). The rate at which particles with velocity $\mathbf{v}$ are produced at the sphere is given by the first term. The kernel $P\left(\mathbf{v}^{\prime}, \mathbf{v}, \hat{\boldsymbol{\sigma}}\right)$ gives the probability that a molecule hitting the sphere at the position $R \hat{\sigma}$ with velocity $\mathbf{v}^{\prime}$ is scattered back with velocity $\mathbf{v}$. It has to satisfy a few conditions: Because of the geometric restrictions imposed on a collision by the sphere, $P\left(\mathbf{v}^{\prime}, \mathbf{v}, \hat{\boldsymbol{\sigma}}\right)$ must be proportional to the product $\Theta(\mathbf{v} \cdot \hat{\sigma}) \Theta\left(-\mathbf{v}^{\prime} \cdot \hat{\sigma}\right)$. Furthermore, $P$ must satisfy the normalization condition

$$
\int d \mathbf{v} P\left(\mathbf{v}^{\prime}, \mathbf{v}, \hat{\boldsymbol{\sigma}}\right)=\Theta\left(-\mathbf{v}^{\prime} \cdot \hat{\boldsymbol{\sigma}}\right)
$$

expressing the conservation of particles in collisions at the surface. Finally, if the gas-sphere system is in equilibrium (which implies that the sphere has zero velocity and zero acceleration with respect to the gas), the solution of the extended Boltzmann equation must be the corresponding MaxwellBoltzmann distribution

$$
f(\mathbf{r}, \mathbf{v}, t)=n W(r) \varphi(v)
$$

where $n$ is the equilibrium density; the overlap function $W$ is given by

and

$$
W(r)=\Theta(r-R)
$$

$$
\varphi(v)=(\beta m / 2 \pi)^{3 / 2} \exp \left(-\beta m v^{2} / 2\right)
$$

where $\left(k_{\mathrm{B}} \beta\right)^{-1}=T$ is the equilibrium temperature and $k_{\mathrm{B}}$ is Boltzmann's constant. 
In that case $P$ has to satisfy the thermostat condition ${ }^{(2,6,7)}$

$$
\int d \mathbf{v}^{\prime} P\left(\mathbf{v}^{\prime}, \mathbf{v}, \hat{\boldsymbol{\sigma}}\right)\left|\mathbf{v}^{\prime} \cdot \hat{\boldsymbol{\sigma}}\right| \varphi\left(v^{\prime}\right)=\Theta(\mathbf{v} \cdot \hat{\boldsymbol{\sigma}})|\mathbf{v} \cdot \hat{\boldsymbol{\sigma}}| \varphi(v)
$$

There are circumstances where the kernel $P\left(\mathbf{v}^{\prime}, \mathbf{v}, \hat{\sigma}\right)$ depends on time or on the distribution function near the wall ; for instance, whenever the wall temperature adjusts itself locally to the gas temperature. Under these circumstances, $P\left(\mathbf{v}^{\prime}, \mathbf{v}, \hat{\boldsymbol{\sigma}}\right)$ may not satisfy the thermostat condition. For simplicity we restrict ourselves here to cases where this is not so, and we assume that $(2.8)$ is satisfied.

In this paper we will mainly be interested either in the case of specular reflection (Fig. 1a), in which the radial component of the velocity of a gas molecule is reversed while the component parallel to the surface of the sphere is not changed, or in the case of diffuse reflection (Fig. 1b), where it is assumed that whenever a particle hits the sphere it is absorbed and immediately reemitted according to a Maxwell-Boltzmann distribution described by a temperature $T_{w}$. The kernel $P$ in these cases has the form

$$
\begin{aligned}
& P_{\mathrm{sp}}\left(\mathbf{v}^{\prime}, \mathbf{v}, \hat{\boldsymbol{\sigma}}\right)=\Theta(\mathbf{v} \cdot \hat{\boldsymbol{\sigma}}) \delta\left(\mathbf{v}-2(\mathbf{v} \cdot \hat{\boldsymbol{\sigma}}) \hat{\boldsymbol{\sigma}}-\mathbf{v}^{\prime}\right) \\
& P_{\mathrm{di}}\left(\mathbf{v}^{\prime}, \mathbf{v}, \hat{\boldsymbol{\sigma}}\right)=\left(2 \pi \beta_{w} m\right)^{\mathbf{1} / 2} \Theta\left(-\mathbf{v}^{\prime} \cdot \hat{\boldsymbol{\sigma}}\right) \Theta(\mathbf{v} \cdot \hat{\boldsymbol{\sigma}})|\mathbf{v} \cdot \hat{\boldsymbol{\sigma}}| \varphi_{w}(v)
\end{aligned}
$$

Here $\beta_{w}=\left(k_{\mathrm{B}} T_{w}\right)^{-1}$ and $\varphi_{w}(v)=\left(\beta_{w} m / 2 \pi\right)^{3 / 2} \exp \left(-\beta_{w} m v^{2} / 2\right)$. One easily checks that (2.9a) satisfies the conditions (2.4) and (2.8) and so does (2.9b) provided $\beta=\beta_{w}$.

Inserting (2.9a) and (2.9b) into (2.3), one obtains the following explicit forms for the action of the specular and diffuse reflection operators, respectively:

$$
\begin{aligned}
\overline{\mathbf{T}}_{\mathrm{sp}} f(\mathbf{r}, \mathbf{v}, t) & \\
= & R^{2} \int d \hat{\boldsymbol{\sigma}}|\mathbf{v} \cdot \hat{\boldsymbol{\sigma}}| \delta(\mathbf{r}-R \hat{\boldsymbol{\sigma}}) \\
& \times\left\{\Theta(\mathbf{v} \cdot \hat{\boldsymbol{\sigma}}) f^{+}[\mathbf{r}, \mathbf{v}-2(\mathbf{v} \cdot \hat{\boldsymbol{\sigma}}) \hat{\boldsymbol{\sigma}}, t]-\Theta(-\mathbf{v} \cdot \hat{\boldsymbol{\sigma}}) f^{+}(\mathbf{r}, \mathbf{v}, t)\right\} \\
\overline{\mathbf{T}}_{\mathrm{di}} f(\mathbf{r}, \mathbf{v}, t) & \\
= & R^{2} \int d \hat{\boldsymbol{\sigma}}|\mathbf{v} \cdot \hat{\boldsymbol{\sigma}}| \delta(\mathbf{r}-R \hat{\boldsymbol{\sigma}}) \\
& \times\left\{\left(2 \pi \beta_{w} m\right)^{1 / 2} \Theta(\mathbf{v} \cdot \hat{\boldsymbol{\sigma}}) \varphi_{w}(v) \int d \mathbf{v}^{\prime} \Theta\left(-\mathbf{v}^{\prime} \cdot \hat{\boldsymbol{\sigma}}\right)\left|\mathbf{v}^{\prime} \cdot \hat{\boldsymbol{\sigma}}\right| f^{+}\left(\mathbf{r}, \mathbf{v}^{\prime}, t\right)\right. \\
& \left.-\Theta(-\mathbf{v} \cdot \hat{\boldsymbol{\sigma}}) f^{+}(\mathbf{r}, \mathbf{v}, t)\right\}
\end{aligned}
$$


Furthermore, we will sometimes consider a linear combination of the specular and the diffuse reflection operators defined by

$$
\overline{\mathbf{T}}_{\alpha}=\alpha \overline{\mathbf{T}}_{\mathrm{di}}+(1-\alpha) \overline{\mathbf{T}}_{\mathrm{sp}}
$$

The coefficient $\alpha$ is called Maxwell's accommodation coefficient. ${ }^{(9)}$

Having defined the $\overline{\mathbf{T}}$ operator for the cases of interest, we point out that the extended Boltzmann equation $(2.1)$ can be viewed as an inhomogeneous Boltzmann equation whose inhomogeneous term is given by $\overline{\mathbf{T}} f$. As such, $\overline{\mathbf{T}} f$ is a somewhat unusual inhomogeneous term since it depends on the distribution function $f$ that we want to determine.

We now show that the extended Boltzmann equation (2.1) is also equivalent to the ordinary Boltzmann equation where the $\overline{\mathbf{T}} f$ term does not appear, but supplemented by boundary conditions that are imposed on the distribution function at the surface of the sphere. To do this we look for solutions of (2.1) which vanish inside the sphere, since no particles should be located there. That is, we look for solutions of the form

$$
f(\mathbf{r}, \mathbf{v}, t)=W(r) \tilde{f}(\mathbf{r}, \mathbf{v}, t)
$$

where $\tilde{f}(\mathbf{r}, \mathbf{v}, t)$ is taken to be continuous as a function of $\mathbf{r}$ as $r \rightarrow R^{+}$. Substituting $f$ given by (2.12) into (2.1) and noting that

$$
\mathbf{v} \cdot \nabla W(r)=(\mathbf{v} \cdot \hat{\mathbf{r}}) \delta(r-R)
$$

where $\hat{\mathbf{r}}=\mathbf{r} / r$, we obtain

with $^{4}$

$$
W(r)\left\{\frac{\partial \tilde{f}}{\partial t}+\mathbf{v} \cdot \mathbf{\nabla} \tilde{f}-\mathbf{a}(t) \cdot \frac{\partial}{\partial \mathbf{v}} \tilde{f}-J(\tilde{f}, \tilde{f})\right\}=\mathbf{T} \tilde{f}
$$

$$
\mathbf{T} \tilde{f}=\overline{\mathbf{T}} \tilde{f}-(\mathbf{v} \cdot \hat{\mathbf{r}}) \delta(r-R) \tilde{f}^{+}
$$

Using (2.10a) and (2.10b), we find that $\mathbf{T}_{\mathrm{sp}}$ and $\mathbf{T}_{\mathrm{di}}$ are given by

$$
\begin{aligned}
& \mathbf{T}_{\mathrm{sp}} \tilde{f}=\Theta(\mathbf{v} \cdot \hat{\mathbf{r}}) \delta(r-R)|\mathbf{v} \cdot \hat{\mathbf{r}}|\left\{\tilde{f}^{+}[\mathbf{r}, \mathbf{v}-2(\mathbf{v} \cdot \hat{\mathbf{r}}) \hat{\mathbf{r}}, t]-\tilde{f}^{+}(\mathbf{r}, \mathbf{v}, t)\right\} \\
& \mathbf{T}_{\mathrm{di}} \tilde{f}= \Theta(\mathbf{v} \cdot \hat{\mathbf{r}}) \delta(r-R)|\mathbf{v} \cdot \hat{\mathbf{r}}|\left\{\varphi_{w}(v)\left(2 \pi \beta_{w} m\right)^{1 / 2}\right. \\
&\left.\times \int d \mathbf{v}^{\prime}\left|\mathbf{v}^{\prime} \cdot \hat{\mathbf{r}}\right| \Theta\left(-\mathbf{v}^{\prime} \cdot \hat{\mathbf{r}}\right) \tilde{f}^{+}\left(\mathbf{r}, \mathbf{v}^{\prime}, t\right)-\tilde{f}^{+}(\mathbf{r}, \mathbf{v}, t)\right\}
\end{aligned}
$$

${ }^{4}$ Notice that, if acting on functions which are discontinuous at the surface of the sphere, the $\mathbf{T}$ operator defined in (2.15) is not identical to the $\mathbf{T}$ operator defined in Ref. 16. The $T$ operator defined here always evaluates the function it acts upon at the outside of the sphere. For continuous functions there is no difference between the $\mathbf{T}$ operator used here and that defined in Ref. 16. 
Since the right-hand side of $(2.14)$ is proportional to $\delta(r-R)$ and $\tilde{f}$ is assumed to be continuous at $r=R$, a solution to this equation can only be found if the right- and left-hand sides vanish identically. The vanishing of the left-hand side requires that $\tilde{f}$ satisfies

$$
\frac{\partial \tilde{f}}{\partial t}+\mathbf{v} \cdot \nabla \tilde{f}-\mathbf{a}(t) \cdot \frac{\partial}{\partial \mathbf{v}} \tilde{f}=J(\tilde{f}, \tilde{f}), \quad r>R
$$

while the vanishing of the right-hand side requires, in the case of specular reflection, that

$\tilde{f}^{+}(\mathbf{r}, \mathbf{v}, t)=\tilde{f}^{+}[\mathbf{r}, \mathbf{v}-2(\mathbf{v} \cdot \hat{\mathbf{r}}) \hat{\mathbf{r}}, t] \quad$ at $r=R^{+} \quad$ for $\quad \mathbf{v} \cdot \hat{\mathbf{r}}>0$

and in the case of diffuse reflection, that

$$
\begin{aligned}
\tilde{f}^{+}(\mathbf{r}, \mathbf{v}, t)=\left(2 \pi \beta_{w} m\right)^{1 / 2} \varphi_{w} \int d \mathbf{v}^{\prime}\left|\mathbf{v}^{\prime} \cdot \hat{\mathbf{r}}\right| \Theta\left(-\mathbf{v}^{\prime} \cdot \hat{\mathbf{r}}\right) \tilde{f}^{+}\left(\mathbf{r}, \mathbf{v}^{\prime}, t\right) \\
\text { at } r=R^{+} \text {for } \mathbf{v} \cdot \hat{\mathbf{r}}>0
\end{aligned}
$$

Equation (2.17) together with the boundary conditions (2.18a) or (2.18b) forms the usual starting point for the kinetic theory of gas flows, ${ }^{(6,7)}$ and we see that this is equivalent to (2.1). We also see from Eqs. (2.12), (2.14), and (2.16a) or (2.16b) that the distribution function $f(\mathbf{r}, \mathbf{v})=$ $n \varphi(v) W(r)$ is indeed the solution of (2.1) that corresponds to the case of total equilibrium.

A similar discussion can be given for cylindrically shaped objects and so on, but we will not elaborate on this here.

\section{NORMAL SOLUTIONS AND THE EXTENDED BOLTZMANN EQUATION}

The usual way of solving the Boltzmann equation in a system without boundaries (either an infinite or a periodic system) is to assume that starting from some initial state, the distribution function of the gas will decay after a few mean free times to a so-called normal solution. ${ }^{(3)}$ Normal solutions vary slowly in space and time, that is, they vary appreciably only on a macroscopic length scale $S$ such that $S \gg l$, the mean free path, and on a time scale $S / c$, where $c$ is the sound velocity. They are completely determined by the hydrodynamic densities, which are the particle density $n(\mathbf{r}, t)$, the energy density [or equivalently the local temperature $T(\mathbf{r}, t)]$, and the momentum density [or equivalently the local velocity $\mathbf{u}(\mathbf{r}, t)$ ]. Furthermore, they can be expressed by an expansion in powers of the gradients in the form

$$
f_{\mathrm{NS}}(\mathbf{r}, \mathbf{v}, t)=f_{0}(\mathbf{r}, \mathbf{v} \mid n, \mathbf{u}, T)+f_{1}(\mathbf{r}, \mathbf{v} \mid n, \mathbf{u}, T)+\cdots
$$


$f_{0}$ is a local equilibrium distribution, given by

$$
\begin{aligned}
f_{0}(\mathbf{r}, \mathbf{v} \mid n, \mathbf{u}, T) & =n(\mathbf{r}, t)[\beta(\mathbf{r}, t) m / 2 \pi]^{3 / 2} \exp \left[-\beta(\mathbf{r}, t) m C^{2}(\mathbf{r}, t) / 2\right] \\
& \equiv n(\mathbf{r}, t) \varphi_{\beta}(C) \equiv f_{0}(\mathbf{r}, C)
\end{aligned}
$$

where $\beta(\mathbf{r}, t)=\left[k_{\mathrm{B}} T(\mathbf{r}, t)\right]^{-1}$ and $\mathbf{C}(\mathbf{r}, t)=\mathbf{v}-\mathbf{u}(\mathbf{r}, t)$. The $f_{1}$ is proportional to gradients of the hydrodynamic variables, and is given by

$$
\begin{aligned}
f_{1}(\mathbf{r}, \mathbf{v} \mid n, \mathbf{u}, T)= & f_{0}\left\{A\left(C^{2}\right) \mathbf{C} \cdot \nabla \log T(\mathbf{r}, t)\right. \\
& \left.+\beta m B\left(C^{2}\right)\left(\mathbf{C C}-\frac{1}{3} C^{2} 1\right): \nabla \mathbf{u}(\mathbf{r}, t)\right\}
\end{aligned}
$$

Here $\mathbb{1}$ is the unit dyadic tensor. The functions $A\left(C^{2}\right)$ and $B\left(C^{2}\right)$ are determined as solutions of the inhomogeneous integral equations

$$
\begin{aligned}
L A\left(C^{2}\right) \mathbf{C} & =\left(\beta m C^{2} / 2-\frac{5}{2}\right) \mathbf{C} \\
L B\left(C^{2}\right)\left(\mathbf{C C}-\frac{1}{3} C^{2} \downarrow\right) & =\mathbf{C C}-\frac{1}{3} C^{2} 1
\end{aligned}
$$

where $L$ is the linearized Boltzmann operator, defined by

$$
\begin{aligned}
L \Phi(\mathbf{r}, \mathbf{C}, t)= & \int d \mathbf{C}_{1} \int_{0}^{a} b d b \int_{0}^{2 \pi} d \varphi\left|\mathbf{C}-\mathbf{C}_{1}\right| f_{0}\left(\mathbf{r}, C_{1}\right) \\
& \times\left\{\Phi\left(\mathbf{r}, \mathbf{C}^{\prime}, t\right)+\Phi\left(\mathbf{r}, \mathbf{C}_{1}{ }^{\prime}, t\right)-\Phi(\mathbf{r}, \mathbf{C}, t)-\Phi\left(\mathbf{r}, \mathbf{C}_{1}, t\right)\right\}
\end{aligned}
$$

This operator has the following important properties: it is symmetric and it is isotropic in C. Furthermore, it is nonnegative definite, it has five zero eigenfunctions, which are the so-called collisional invariants, $1, \mathbf{C}$, and $C^{2}$ (or equivalently $1, \mathbf{v}$, and $v^{2}$ ) and the spectrum of $L$ has a gap between zero and the set of nonzero eigenvalues. ${ }^{(6)}$

Uniqueness of the solutions of (3.4a) and (3.4b) is obtained by requiring that the functions on the left-hand side are orthogonal to the zero eigenfunctions of $L$. The only resulting condition which is not satisfied automatically as a consequence of isotropy is

$$
\int d \mathbf{C} f_{0}(C) C^{2} A\left(C^{2}\right)=0
$$

By inserting the normal solution (3.1) into the Boltzmann equation, multiplying by $1, \mathbf{v}$, or $v^{2}$, and integrating over all velocities, one obtains the hydrodynamic equations in a form depending on the order of truncation of (3.1). For example, keeping only $f_{0}$, one obtains the Euler equations. Keeping both $f_{0}$ and $f_{1}$, one obtains the Navier-Stokes equations,

$$
\begin{gathered}
\partial n / \partial t+\nabla \cdot n \mathbf{u}=0 \\
m n(\partial \mathbf{u} / \partial t+\mathbf{u} \cdot \nabla \mathbf{u})=n \mathbf{F}-\nabla\left(n k_{\mathrm{B}} T\right)+\nabla \cdot\left\{2 n\left[\mathrm{D}-\frac{1}{3}(\nabla \cdot \mathbf{u})\right]\right\} \\
\frac{3}{2} n k_{\mathrm{B}}(\partial T / \partial t+\mathbf{u} \cdot \nabla T)+n k_{\mathrm{B}} T \nabla \cdot \mathbf{u}=\nabla \cdot \lambda \nabla T+2 \eta\left[\mathrm{D}: \mathrm{D}-\frac{1}{3}(\nabla \cdot \mathbf{u})^{2}\right]
\end{gathered}
$$


Here $\mathbf{F}$ is an external force acting on the particles. In our case $\mathbf{F}$ is a virtual external force, given as $\mathbf{F}=-m \mathbf{a}(\mathbf{r}, t)$. The rate of strain tensor $\mathrm{D}$ has components given by

$$
D_{i j}=\frac{1}{2}\left(\partial u_{i} / \partial x_{j}+\partial u_{j} / \partial x_{i}\right)
$$

The shear viscosity $\eta$ and the heat conductivity $\lambda$ follow from the Boltzmann equation as

$$
\begin{aligned}
& \eta=\left\{-m^{2} /\left[15 k_{\mathrm{B}} T(\mathrm{r}, t)\right]\right\} \int d \mathbf{C} f_{0}(C) C^{4} B\left(C^{2}\right) \\
& \lambda=-{ }_{3} k_{\mathrm{B}} \int d \mathrm{C} f_{0}(C)\left(\beta m C^{2} / 2-\frac{5}{2}\right) C^{2} A\left(C^{2}\right)
\end{aligned}
$$

Now the question arises whether the same normal solutions satisfy the extended Boltzmann equation describing a system in contact with its walls. We will presently see that in general this is not the case, since it is well known that close to the boundaries there exist kinetic boundary layers as a result of the specific interactions between the gas molecules and the walls. ${ }^{(6,7)}$ Then the next question is whether the normal solution is an approximate solution of the extended Boltzmann equation, and, if so, to what order of approximation. We will demonstrate how this problem can be attacked formally in the case of flow past a sphere and we will show how the boundary conditions on the hydrodynamic equations are obtained to leading order in the Knudsen number $\mathscr{K}=l / R$.

Does there exist a normal solution of the form (3.1) satisfying the extended Boltzmann equation (2.1), or equivalently, Eqs. (2.17) and (2.18)? Since the expansion (3.1) is a solution of the ordinary Boltzmann equation (2.17), we need only be concerned about (2.18). Consider first the case of diffuse reflection. According to (2.16b), the action of $\mathbf{T}_{\mathrm{di}}$ on $f_{0}$ and $f_{1}$, respectively, is given as

$$
\begin{aligned}
\mathbf{T}_{\mathrm{di}} f_{0}= & \Theta(\mathbf{v} \cdot \hat{\mathbf{r}})|\mathbf{v} \cdot \hat{\mathbf{r}}| \delta(r-R) n(\mathbf{r}, t) \\
& \times\left\{\left(\beta_{w} m / 2 \pi\right)^{3 / 2} \exp \left(-\beta_{w} m v^{2} / 2\right)\left(2 \pi \beta_{w} m\right)^{1 / 2} \int d \mathbf{v}^{\prime}\left|\mathbf{v}^{\prime} \cdot \hat{\mathbf{r}}\right| \Theta\left(-\mathbf{v}^{\prime} \cdot \hat{\mathbf{r}}\right)\right. \\
& \times(\beta m / 2 \pi)^{3 / 2} \exp \left(-\beta m C^{\prime 2} / 2\right) \\
& \left.-(\beta m / 2 \pi)^{3 / 2} \exp \left(-\beta m C^{2} / 2\right)\right\}
\end{aligned}
$$




$$
\begin{aligned}
\mathbf{T}_{\mathrm{di}} f_{1}= & \Theta(\mathbf{v} \cdot \hat{\mathbf{r}})|\mathbf{v} \cdot \hat{\mathbf{r}}| \delta(r-R) n(\mathbf{r}, t) \\
& \times\left\{\left(\beta_{w} m / 2 \pi\right)^{3 / 2}\left[\exp \left(-\beta_{w} m v^{2} / 2\right)\right]\left(2 \pi \beta_{w} m\right)^{1 / 2}\right. \\
& \times \int d \mathbf{v}^{\prime}\left|\mathbf{v}^{\prime} \cdot \hat{\mathbf{r}}\right| \Theta\left(-\mathbf{v}^{\prime} \cdot \hat{\mathbf{r}}\right)(\beta m / 2 \pi)^{3 / 2}\left[\exp \left(-\beta m C^{\prime 2} / 2\right)\right] \\
& \times\left[A\left(C^{\prime 2}\right) \mathbf{C}^{\prime} \cdot \nabla \log T+\beta m B\left(C^{\prime 2}\right)\left(\mathbf{C}^{\prime} \mathbf{C}^{\prime}-\frac{1}{3} C^{\prime 2} 1\right): \mathbf{v u}\right] \\
& -(\beta m / 2 \pi)^{3 / 2}\left[\exp \left(-\beta m C^{2} / 2\right)\right] \\
& \left.\times\left[A\left(C^{2}\right) \mathbf{C} \cdot \mathbf{\nabla} \log T+\beta m B\left(C^{2}\right)\left(\mathbf{C C}-\frac{1}{3} C^{2} \mathfrak{0}\right): \nabla \mathbf{u}\right]\right\}
\end{aligned}
$$

If $f$ is approximated by $f_{0}$ alone, the condition $\mathbf{T}_{\mathrm{di}} f_{0}=0$ gives rise to hydrodynamic stick boundary conditions

$$
\mathbf{u}(\mathbf{r}, t)=0, \quad \beta(\mathbf{r}, t)=\beta_{w} \quad \text { for } r=R
$$

Together with boundary conditions at infinity, these are sufficient to determine a solution of the hydrodynamic equations. They lead, for instance, to Stokes' result $\mathbf{F}=-6 \pi \eta R \mathbf{V}$ for the drag force on a sphere of radius $R$ moving through a fluid with shear viscosity $\eta$ at a constant velocity $V$. If the approximation for $f$ is extended to $f=f_{0}+f_{1}$, the condition $\mathbf{T}_{\mathrm{di}}\left(f_{0}+f_{1}\right)$ separates into two conditions $\mathbf{T}_{\mathrm{di}} f_{0}=0$ and $\mathbf{T}_{\mathrm{di}} f_{1}=0$, because $\mathbf{T}_{\mathrm{di}} f_{0}$ and $\mathbf{T}_{\mathrm{di}} f_{1}$ are linearly independent functions of the velocity. But the condition $\mathbf{T}_{\mathrm{di}} f_{1}=0$ cannot be satisfied at the same time as $\mathbf{T}_{\mathrm{di}} f_{0}=0$, since it requires that not only the local velocity itself, but also all its first spatial derivatives vanish at the boundary, which is not in agreement with the known solutions of the hydrodynamic equations. Addition of $f_{2}, f_{3}$, etc. to the approximation for $f$ makes matters worse, because each $\mathbf{T}_{\mathrm{di}} f_{i}$ is linearly independent of all the previous ones, so each added term requires new boundary conditions to be fulfilled simultaneously with the ones obtained already.

Hence we must conclude that the normal solution alone does not satisfy the extended Boltzmann equation. Instead, this equation will have solutions consisting of a normal part and a nonnormal part, which adjusts the total solution to the conditions imposed by the boundary. Since the nonnormal part is generated by the boundary, it may be expected to vanish exponentially within a distance of a few mean free paths from the walls. Because inconsistencies between the hydrodynamic boundary conditions and the requirement $\mathbf{T} f=0$ show up only if $f$ is expanded through order $l / R$ ( $f_{1}$ is of this order relative to $f_{0}$ ), the nonnormal or boundary layer part of the solution will presumably also be of order $l / R$. Hence the boundary conditions will 
also be affected in order $l / R$ only and to leading order in the Knudsen number the stick boundary conditions (3.11) will still be correct.

Let us next turn to specular boundary conditions. Here the action of $\mathbf{T}_{\mathrm{sp}}$ on the normal solution is given as

$$
\begin{aligned}
& \mathbf{T}_{\mathrm{sp}} f_{0}=n(\beta m / 2 \pi)^{3 / 2} \Theta(\mathbf{v} \cdot \hat{\mathbf{r}})|\mathbf{v} \cdot \hat{\mathbf{r}}| \delta(r-R) \\
& \times\left[\exp \left(-\beta m C^{\prime 2} / 2\right)-\exp \left(-\beta m C^{2} / 2\right)\right] \\
& \mathbf{T}_{\mathrm{sp}} f_{1}=n(\beta m / 2 \pi)^{3 / 2} \Theta(\mathbf{v} \cdot \hat{\mathbf{r}})|\mathbf{v} \cdot \hat{\mathbf{r}}| \delta(r-R) \\
& \times\left\{[ \operatorname { e x p } ( - \beta m C ^ { \prime 2 } / 2 ) ] \left[A\left(C^{\prime 2}\right) \mathbf{C}^{\prime} \cdot \nabla \log T+\beta m B\left(C^{\prime 2}\right)\right.\right. \\
& \left.\times\left(\mathbf{C}^{\prime} \mathbf{C}^{\prime}-\frac{1}{3} C^{\prime 2} \rrbracket\right): \nabla \mathbf{v}\right]-\left[\exp \left(-\frac{1}{2} \beta m C^{2}\right)\right] \\
& \left.\times\left[A\left(C^{2}\right) \mathbf{C} \cdot \boldsymbol{\nabla} \log T+\beta m B\left(C^{2}\right)\left(\mathbf{C C}-\frac{1}{3} C^{2} 1\right): \nabla \mathbf{u}\right]\right\}
\end{aligned}
$$

where $\mathbf{C}^{\prime}=\mathbf{v}-2(\mathbf{v} \cdot \hat{\mathbf{r}}) \hat{\mathbf{r}}-\mathbf{u}(\mathbf{r}, t)$. The requirement that the rhs of $(3.12 \mathrm{a})$ is equal to zero leads to the hydrodynamic boundary condition $\mathbf{u} \cdot \hat{\mathbf{r}}=0$ only. This is of course not sufficient to determine a solution of the hydrodynamic equations. However, addition of the boundary conditions resulting from the condition $\mathbf{T}_{\mathrm{sp}} f_{1}=0$ leads to the following sufficient set of boundary conditions:

$$
\begin{aligned}
\mathbf{u} \cdot \hat{\mathbf{r}} & =0 \\
\hat{\mathbf{r}} \cdot \nabla \log T & =0 \quad \text { for } r=R \\
\left(\hat{\mathbf{r}} \hat{\mathbf{s}}^{i}+\hat{\mathbf{s}} i \hat{\mathbf{r}}\right): \nabla \mathbf{u} & =0
\end{aligned}
$$

Here $\hat{\mathbf{s}}^{i}$ is any unit vector perpendicular to $\hat{\mathbf{r}}$. The boundary condition $(3,13 \mathrm{c})$ expresses that in the case of specular reflection no tangential stresses are exerted on the sphere. This is what one would expect, since in a specular collision between a gas molecule and the sphere no transfer of momentum directed parallel to the surface takes place. ${ }^{(17)}$

Thus it appears that the case of specular reflection does allow for a normal solution to satisfy the extended Boltzmann equation to order $l / R$. If one extends the expansion of the normal solution to second order, one runs into inconsistencies, exactly as in the diffuse reflection case. The fact that these inconsistencies show up only at order $(l / R)^{2}$ means, however, that the boundary layer effects show up only in the order $(l / R)^{2}$, and also that corrections to the boundary conditions (3.13) are of that order.

One important consequence of the fact that the normal solution of the Boltzmann equation breaks down near the surface of the sphere is that the normal solution cannot in general be used to directly determine the force 
on the sphere. To see this, we use the fact that the drag force $\mathbf{F}(t)$ exerted by the gas molecule on the sphere at time $t$ is given by

$$
\mathbf{F}(t)=-\int d \mathbf{r} \int d \mathbf{v} m \mathbf{v} \overline{\mathbf{T}} f(\mathbf{r}, \mathbf{v}, t)
$$

This equation is easily understood by noting that $\overline{\mathbf{T}} f(\mathbf{r}, \mathbf{v}, t)$ is the rate of change of the distribution function due to collisions between gas molecules and the sphere. Then, by multiplying $\overline{\mathbf{T}} f$ by $m \mathbf{v}$ and integrating over all $\mathbf{r}$ and $\mathbf{v}$ one obtains the total rate of momentum transfer from the sphere to the gas at time $t$. Now, since $\overline{\mathbf{T}} f(\mathbf{r}, \mathbf{v}, t)$ requires that $f$ be evaluated at the surface of the sphere, and since the normal form of the distribution function is not a solution of the Boltzmann equation near the surface of the sphere, it is clear that $\mathbf{F}(t)$ cannot generally be evaluated from (3.14) using only the normal part of the complete distribution function. For diffuse reflection, in particular, one does not obtain the proper form of Stokes' law $\mathbf{F}=-6 \pi \eta R \mathbf{V}$ from (3.14) using the normal form for $f$ given by Eq. (3.1), but instead one obtains $\mathbf{F}=-4 \pi \eta R \mathbf{V}$. The discrepancy can be accounted for by noting that for diffuse reflection the boundary layer corrections to $f_{\mathrm{NS}}$ are of order $l / R$, and these corrections are needed to obtain the proper form for Stokes' law. ${ }^{5}$ For the case of specular reflection, the normal solution of Eq. (3.1), when inserted in Eq. (3.14), does lead to the correct form $\mathbf{F}=-4 \pi \eta R \mathbf{V}$, since the normal solution is correct to order $(l / R)^{2}$ for this case.

In the following sections we show how one can formally determine the boundary layer part of the distribution function and use it together with the normal part of the distribution function to determine the correct form for the drag force on the sphere from Eq. (3.14) for small values of $l / R$.

\section{LINEARIZATION OF THE EXTENDED BOLTZMANN EQUATION AND PROJECTION ON NORMAL MODES}

Since the normal solution alone does not satisfy the extended Boltzmann equation, we have to construct in some way the complete solution of the Boltzmann equation that: (1) reduces to the usual normal solution far from the sphere, (2) describes the boundary layer correctly, and (3) vanishes in the interior of the sphere.

\footnotetext{
${ }^{5}$ There is no contradiction between this result and the usual, correct result derived from the Navier-Stokes equations, which, in turn, follow from the Chapman-Enskog normal solution. In the hydrodynamic derivation one considers the momentum flux through a surface surrounding the sphere, but outside the boundary layer. The normal solution is valid near this surface and correctly predicts the momentum flux. In Eq. (3.14) one considers the momentum transfer from the gas to the sphere. Here the normal solution and the boundary layer correction both contribute to the momentum flux at the same order in $l / R$.
} 
Ultimately we are interested in the drag force on the sphere, which we expect to be linearly proportional to the velocity of the sphere, provided the latter is sufficiently small. ${ }^{6}$ In that case the extended Boltzmann equation can be linearized in the velocity of the sphere. This linearization allows us to apply a projection operator method in a fairly straightforward manner when we attempt to decompose the distribution function into a normal solution part and a boundary layer part.

As before, the gas-sphere system is considered in a coordinate system where the sphere is at rest. In this frame the gas far from the sphere is in an equilibrium state with uniform velocity $-\mathbf{V}$, where $\mathbf{V}$ is the velocity of the sphere in the laboratory frame. Hence we can write the distribution function of the gas particles as

$$
f(\mathbf{r}, \mathbf{v}, t)=n\left(\frac{\beta m}{2 T}\right)^{3 / 2} \exp \left[-\frac{\beta m}{2}|\mathbf{v}+\mathbf{V}(t)|^{2}\right][W(r)+\Psi(\mathbf{r}, \mathbf{v}, t)]
$$

As before, $n$ and $k_{\mathrm{B}} \beta$ are the equilibrium density and inverse temperature of the gas. The function $\Psi$ has to vanish as $r \rightarrow \infty$. It also must vanish for $r$ inside the sphere, but we consider $f$ and $\Psi$ as functions defined over all space. For later purposes it will be useful to write ${ }^{\prime}$ ' as the sum of an exterior and an interior part,

$$
\Psi(\mathbf{r}, \mathbf{v}, t)=\Theta(r-R) \Psi^{\text {ext }}(\mathbf{r}, \mathbf{v}, t)+\Theta(R-r) \Psi^{\text {int }}(\mathbf{r}, \mathbf{v}, t)
$$

where in fact. $\Psi^{\text {int }}=0$. Insertion of (4.1) into the extended Boltzmann equation (2.1) yields

$$
\begin{aligned}
& n\left(\frac{\beta m}{2 \pi}\right)^{3 / 2} \exp \left[-\frac{\beta m}{2}|\mathbf{v}+\mathbf{V}(t)|^{2}\right] \\
& \quad \times\left\{\left[\frac{\partial}{\partial t}+\mathbf{v} \cdot \nabla-\mathbf{a}(t) \cdot \frac{\partial}{\partial \mathbf{v}}\right] \Psi(\mathbf{r}, \mathbf{v}, t)+\mathbf{v} \cdot \nabla W(r)\right\}=J(f, f)+\overline{\mathbf{T}} f
\end{aligned}
$$

As the next step, (4.3) is linearized in $V$. We assume that $\Psi$ is proportional to $\mathbf{V}$ and neglect all terms in (4.3) of quadratic and higher order in $\mathbf{V}$, including $\mathbf{a} \cdot(\partial / \partial \mathbf{V}) \Psi$. Furthermore, (4.3) is divided by $n \varphi$, where $\varphi(v)$ is given by (2.7). In this way Eq. (4.3) reduces to

$$
\begin{aligned}
\left(\frac{\partial}{\partial t}+\right. & \left.\mathbf{v} \cdot \nabla-L-\overline{\mathbf{T}}^{\prime}\right) \Psi(\mathbf{r}, \mathbf{v}, t) \\
= & -\overline{\mathbf{T}}^{\prime} \beta m[\mathbf{v} \cdot \mathbf{V}(t)]+\alpha \mathbf{T}_{\mathrm{d}}^{\prime}\left(\frac{\beta_{w}-\beta}{\beta}\right)\left(\frac{\beta m v^{2}}{2}-\frac{3}{2}\right) \\
& +\beta m \mathbf{v} \cdot \mathbf{V}(t)[\mathbf{v} \cdot \nabla W(r)]
\end{aligned}
$$

${ }^{6}$ This means that the Reynolds number $(V / c) R / l$ is small. 


$$
=-\mathbf{T}^{\prime}\left[\beta m \mathbf{v} \cdot \mathbf{V}(t)-\frac{\beta_{w}-\beta}{\beta}\left(\frac{\beta m v^{2}}{2}-\frac{3}{2}\right)\right]
$$

where $\overline{\mathbf{T}}$ is given by $(2.10)-(2.11)$ and

$$
\mathbf{T}^{\prime}=[\varphi(v)]^{-1} \overline{\mathbf{T}} \varphi(v)
$$

and $\mathbf{T}^{\prime}$ is defined in an analogous way. We drop the primes again since we always will deal with Eq. (4.4). The term with $\alpha \mathbf{T}_{\mathrm{di}}^{\prime}$ acting on $\beta m v^{2} / 2-\frac{3}{2}$ results from linearization of the actual $\mathbf{T}_{\mathrm{di}}$ about $\mathbf{T}_{\mathrm{di}}\left(\beta_{w}=\beta\right)$. The operator $L$ on the left-hand side of (4.4) is the linearized Boltzmann operator, defined by (3.5) with $\mathbf{C}$ replaced by $\mathbf{v}$ and $n(\mathbf{r}, t)$ by $n$. Again, for mathematical convenience we will assume that (4.4) also holds for the inside of the sphere (which is physically not true), but in the end we will keep only solutions with $\Psi=0$ inside.

The solutions of (4.4) can be analyzed by separating $\Psi$ into a ChapmanEnskog normal solution part and a boundary layer part. The form of the normal solutions of the ordinary linearized Boltzmann equation is obtained by linearizing (3.1) about $n \varphi(v)$ :

$$
\begin{aligned}
f_{\mathrm{NS}}(\mathbf{r}, \mathbf{v}, t)= & n \varphi(v)\left[1+\frac{\delta n(\mathbf{r}, t)}{n}+\beta m \mathbf{v} \cdot \mathbf{u}(\mathbf{r}, t)\right. \\
& +\left(\frac{\beta m}{2} v^{2}-\frac{3}{2}\right) \frac{\delta T(\mathbf{r}, t)}{T}+A\left(v^{2}\right) \mathbf{v} \cdot \nabla \frac{\delta T(\mathbf{r}, t)}{T} \\
& \left.+\beta m B\left(v^{2}\right)\left(\mathbf{v v}-\frac{1}{3} v^{2} \emptyset\right): \nabla \mathbf{u}(\mathbf{r}, t)+O\left(\nabla^{2}\right)\right]
\end{aligned}
$$

Here $\delta n(\mathbf{r}, t)$ and $\delta T(\mathbf{r}, t)$ are the deviations of the local density and temperature from their equilibrium values $n$ and $T$. The functions $A$ and $B$ are determined through Eq. (3.4). It follows from the linearized Boltzmann equation that the fluid fields $\delta n, \delta T$, and $\mathbf{u}$ satisfy the linearized hydrodynamic equations with constant transport coefficients given by (3.9) with fixed $\beta$, and they vary on a length scale large compared to the mean free path.

The normal solutions (4.6) can be written in terms of the so-called hydrodynamic mode eigenfunctions of the operator $-\mathbf{v} \cdot \nabla+L$. These eigenfunctions depend on a wave number $\mathbf{k}$, which is restricted to satisfy $k l \ll 1$, where $l$ is a mean free path length. ${ }^{(10.11,18)}$ In the limit $k \rightarrow 0$ the hydrodynamic modes approach linear combinations of collisional invariants and their eigenvalues approach zero, at least proportionally to $k$. In three dimensions there are two shear modes, two sound modes, and one heat mode for each value of $\mathbf{k}$, given respectively as ${ }^{(10)}$

$$
\begin{aligned}
\Psi_{\mathbf{k}}{ }^{j}(\mathbf{r}, \mathbf{v})= & {[\exp (i \mathbf{k} \cdot \mathbf{r})](\beta m)^{1 / 2} } \\
& \times\left[\mathbf{v} \cdot \hat{\mathbf{k}}_{\perp}^{j}+i B\left(v^{2}\right)\left(\mathbf{v v}-\frac{1}{3} v^{2} \uparrow\right): \mathbf{k} \hat{\mathbf{k}}_{\perp}^{j}+O\left(k^{2}\right)\right]
\end{aligned}
$$




$$
\begin{aligned}
\Psi_{\mathbf{k}}{ }^{\sigma}(\mathbf{r}, \mathbf{v})= & {[\exp (i \mathbf{k} \cdot \mathbf{r})]\left[\sigma\left(\frac{\beta m}{2}\right)^{1 / 2}(\mathbf{v} \cdot \hat{\mathbf{k}})+\frac{1}{\sqrt{30}} \beta m v^{2}\right.} \\
& +i k\left\{\left(\frac{2 v-D_{T}}{6 c}\right)\left[\frac{\sigma}{\sqrt{30}} \beta m v^{2}-\left(\frac{\beta m}{2}\right)^{1 / 2}(\mathbf{v} \cdot \hat{\mathbf{k}})\right]\right. \\
& -\frac{\sigma D_{T}}{c \sqrt{30}}\left(\beta m v^{2}-5\right)+\sigma\left(\frac{\beta m}{2}\right)^{1 / 2} B\left(v^{2}\right)\left[(\mathbf{v} \cdot \hat{\mathbf{k}})^{2}-\frac{1}{3} v^{2}\right] \\
& \left.\left.+\frac{2}{\sqrt{30}} A\left(v^{2}\right)(\mathbf{v} \cdot \hat{\mathbf{k}})\right\}+O\left(k^{2}\right)\right] \\
\Psi_{\mathbf{k}}{ }^{H}(\mathbf{r}, \mathbf{v})= & {[\exp (i \mathbf{k} \cdot \mathbf{r})]\left\{-\frac{1}{\sqrt{10}}\left(\beta m v^{2}-5\right)+i k\right.} \\
& \left.\times\left[\frac{D_{T}}{c}\left(\frac{2}{5} \beta m\right)^{1 / 2}(\mathbf{v} \cdot \hat{\mathbf{k}})+\frac{2}{\sqrt{10}} A\left(v^{2}\right)(\mathbf{v} \cdot \hat{\mathbf{k}})\right]+O\left(k^{2}\right)\right\}
\end{aligned}
$$

In (4.7a), $\hat{\mathbf{k}}_{\perp}{ }^{1}$ and $\hat{\mathbf{k}}_{\perp}{ }^{2}$ are two unit vectors orthogonal to $\hat{\mathbf{k}}$ and to each other. In (4.7b) and (4.7c), $c=\left(\frac{3}{5} \beta m\right)^{-1 / 2}$ is the speed of sound, $v=\eta / \mathrm{nm}$ is the kinematic viscosity, and $D_{T}=\lambda / n C_{p}$ is the thermal diffusivity. $\sigma$ is either +1 or -1 . The eigenvalues to order $k^{2}$ of shear modes, sound modes, and the heat mode, respectively, are $-v k^{2},-\left(\sigma i c k+\frac{1}{2} \Gamma k^{2}\right)$, and $-D_{T} k^{2}$, where the sound wave damping constant $\Gamma=\frac{4}{3} v+\frac{2}{3} D_{T}$. Contributions to the hydrodynamic modes of $O\left(k^{2}\right)$ and to the hydrodynamic eigenvalues of $O\left(k^{3}\right)$ are neglected consistently. There is a natural wave number $k_{0}$, on the order of the inverse mean free path, above which the expansion of eigenfunctions and eigenvalues in powers of $k$ breaks down and hydrodynamic modes no longer exist.

After introducing the inner product

$$
\langle f(\mathbf{r}, \mathbf{v}) \mid g(\mathbf{r}, \mathbf{v})\rangle=\int d \mathbf{r} \int d \mathbf{v} \varphi(v) f^{*}(\mathbf{r}, \mathbf{v}) g(\mathbf{r}, \mathbf{v})
$$

we find the adjoint of $-\mathbf{v} \cdot \nabla+L$ to be $\mathbf{v} \cdot \nabla+L$, since both $\mathrm{v} \cdot \nabla$ and $L$ commute with $\varphi(v)$. Since the action of $\mathbf{v} \cdot \nabla$ on the hydrodynamic modes (4.7) is simply a multiplication by $i \mathbf{k} \cdot \mathbf{v}$ and $L$ is a real operator, one sees that the right eigenfunctions of $\mathbf{v} \cdot \nabla+L$ (which are the left eigenfunctions of $-\mathbf{v} \cdot \boldsymbol{\nabla}+L$ ) are obtained as

$$
\varphi_{\mathbf{k}}{ }^{\gamma}(\mathbf{r}, \mathbf{v})=[\exp (i \mathbf{k} \cdot \mathbf{r})]\left\{[\exp (-i \mathbf{k} \cdot \mathbf{r})] \Psi_{\mathbf{k}}^{\gamma}(\mathbf{r}, \mathbf{v})\right\}^{*}
$$

Now if $n \varphi(v) \Psi$ is a solution of the linearized Boltzmann equation, the 
normal part of $\Psi$ is obtained by a projection onto the hydrodynamic modes, i.e.,

$$
P \Psi(\mathbf{r}, \mathbf{v}, t)=\frac{1}{(2 \pi)^{3}} \int_{k<k_{0}} d \mathbf{k} \sum_{\nu}\left|\Psi_{\mathbf{k}}^{\gamma}(\mathbf{r}, \mathbf{v})\right\rangle\left\langle\varphi_{\mathbf{k}}^{\gamma^{*}}(\mathbf{r}, \mathbf{v}) \mid \Psi(\mathbf{r}, \mathbf{v}, t)\right\rangle
$$

and $\gamma$ runs over the five types of hydrodynamic modes. The form of this projection operator can be simplified appreciably. First it is possible to perform a rotation among the hydrodynamic modes for one given value of k and replace them by a "density mode," a "temperature mode," and three "velocity modes." These are given as

$$
\begin{aligned}
& \Psi_{\mathbf{k}}{ }^{n}=\exp (i \mathbf{k} \cdot \mathbf{r}) \\
& \Psi_{\mathbf{k}}{ }^{T}=[\exp (i \mathbf{k} \cdot \mathbf{r})]\left(\frac{2}{3}\right)^{1 / 2}\left[\frac{1}{2} \beta m v^{2}-\frac{3}{2}+i \mathbf{k} \cdot \mathbf{v} A\left(v^{2}\right)+O\left(k^{2}\right)\right]
\end{aligned}
$$

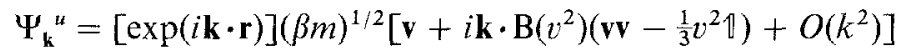

The corresponding "left modes" $\varphi_{\mathbf{k}}{ }^{T}$ and $\varphi_{\mathbf{k}}{ }^{\mu}$ are obtained by replacing $i$ by $-i$ in the brackets in (4.11b) and (4.11c), while $\varphi_{\mathbf{k}}{ }^{n}=\Psi_{\mathbf{k}}{ }^{n}$. These functions are no longer eigenfunctions of the operator $-\mathbf{v} \cdot \nabla+L$, but they do span the space of normal solutions just as well. A further simplification is obtained by actually performing the integrations over $\mathbf{k}$ in (4.10). These integrations give rise to smeared-out $\delta$-functions, e.g.,

$$
\begin{aligned}
\frac{1}{(2 \pi)^{3}} \int d \mathbf{k}\left|\Psi_{\mathbf{k}}{ }^{n}\right\rangle\left\langle\varphi_{\mathbf{k}}{ }^{n}\right| & =\frac{1}{(2 \pi)^{3}} \int_{k<k_{0}} d \mathbf{k} \exp (i \mathbf{k} \cdot \mathbf{r}) \int d \mathbf{r}^{\prime} \exp \left(-i \mathbf{k} \cdot \mathbf{r}^{\prime}\right) \\
& =\int d \mathbf{r}^{\prime} \Delta_{k_{0}}\left(\mathbf{r}-\mathbf{r}^{\prime}\right)
\end{aligned}
$$

The smearing out of the $\delta$-function is due to the large- $k$ cutoff in the integration and takes place over a volume of diameter $\sim 1 / k_{0} \approx l$ centered at $\mathbf{r}$. As long as the projection operator acts on functions which are smooth on the length scale of the mean free path, the integrals over the $\Delta_{k_{0}}$ functions can all be done, which reduces the projection operator to

$$
\left.P=\mid \Psi^{n}(\mathbf{v})\right)\left(\Psi ^ { n } ( \mathbf { v } ) | + | \Psi ^ { T } ( \mathbf { v } ) ( \Psi ^ { T } ( \mathbf { v } ) | + | \Psi ^ { u } ( \mathbf { v } ) ) \cdot \left(\Psi^{u}(\mathbf{v}) \mid\right.\right.
$$

The parentheses now denote an inner product in velocity space, defined by

$$
(f(\mathbf{v}) \mid g(\mathbf{v}))=\int d \mathbf{v} \varphi(v) f(\mathbf{v}) g(\mathbf{v})
$$

Note that the complex conjugate of $f$ is not taken. Furthermore, the 
"functions" $f$ and $g$ may contain operators, such as a spatial gradient. The $\Psi$ 's in (4.13) are given as

$$
\begin{aligned}
& \Psi^{n}(\mathbf{v})=1 \\
& \Psi^{T}(\mathbf{v})=\left(\frac{2}{3}\right)^{1 / 2}\left[\frac{1}{2} \beta m v^{2}-\frac{3}{2}+A\left(v^{2}\right) \mathbf{v} \cdot \nabla+\cdots\right] \\
& \Psi^{u \prime}(\mathbf{v})=(\beta m)^{1 / 2}\left[\mathbf{v}+B\left(v^{2}\right)\left(\mathbf{v} \mathbf{v}-\frac{1}{3} v^{2} \|\right) \cdot \nabla+\cdots\right]
\end{aligned}
$$

One easily checks that the linearized normal solution (4.6) can be written in terms of these functions as

$$
\left.\left.\left.\Psi_{\mathrm{NS}}=\left[\mid \Psi^{n}\right) \delta n / n+\left(\frac{3}{2}\right)^{1 / 2} \mid \Psi^{T}\right) \delta T / T+(\beta m)^{1 / 2} \mid \Psi^{u}\right) \cdot \mathbf{u}\right]
$$

It is clear that application of $P$ given by (4.13) to $\Psi_{\mathrm{NS}}$ indeed reproduces the normal solutions. The large- $k$ cutoff in (4.12) implies, however, that (4.13) is only valid when acting on functions with not too steep gradients; this means that the typical length of the gradients must be $\gg l$.

If we now make the separation

$$
\Psi(\mathbf{r}, \mathbf{v}, t)=P \Psi(\mathbf{r}, \mathbf{v}, t)+P_{\perp} \Psi(\mathbf{r}, \mathbf{v}, t)
$$

then (4.4) can be replaced by two coupled equations for $P \Psi$ and $P_{\perp} \Psi$, respectively,

$$
\begin{aligned}
& P\left(\frac{\partial}{\partial t}+\mathbf{v} \cdot \nabla-L-\overline{\mathbf{T}}\right) P \Psi \\
& =P \overline{\mathbf{T}} P_{\perp} \Psi-P \mathbf{T}\left[\beta m \mathbf{v} \cdot \mathbf{V}(t)-\frac{\beta_{w}-\beta}{\beta}\left(\frac{\beta m}{2} v^{2}-\frac{3}{2}\right)\right] \\
& P_{\perp}\left(\frac{\partial}{\partial t}+\mathbf{v} \cdot \boldsymbol{\nabla}-L-\overline{\mathbf{T}}\right) P_{\perp} \Psi \\
& =P_{\perp} \overline{\mathbf{T}} P \Psi-P_{\perp} \mathbf{T}\left[\beta m \mathbf{v} \cdot \mathbf{V}(t)-\frac{\beta_{w}-\beta}{\beta}\left(\frac{\beta m}{2} v^{2}-\frac{3}{2}\right)\right]
\end{aligned}
$$

where we used that $P_{\perp}(\mathbf{v} \cdot \nabla-L) P=P(\mathbf{v} \cdot \nabla-L) P_{\perp}=0$. Equation (4.18b) can be formally solved as

$$
\begin{aligned}
P_{\perp} \Psi(\mathbf{r}, \mathbf{v}, t) \\
=\int_{0}^{t} d \tau \exp \left[-P_{\perp}(\mathbf{v} \cdot \nabla-L-\overline{\mathbf{T}}) P_{\perp} \tau\right] \\
\quad \times P_{\perp}\left\{\overline{\mathbf{T}} P \Psi(\mathbf{r}, \mathbf{v}, t-\tau)-\mathbf{T}\left[\beta m \mathbf{v} \cdot \mathbf{V}(t-\tau)-\frac{\beta_{w}-\beta}{\beta}\left(\frac{\beta m}{2} v^{2}-\frac{3}{2}\right)\right]\right\} \\
\quad+\exp \left[-P_{\perp}(\mathbf{v} \cdot \nabla-L-\overline{\mathbf{T}}) P_{\perp} t\right] P_{\perp} \Psi(\mathbf{r}, \mathbf{v}, 0)
\end{aligned}
$$


Although this solution is of little use for a detailed numerical description of the boundary layer, it does provide some qualitative information on its structure. The exponential operator on the right-hand side must decay to zero on the time scale of the mean free time. This has been shown for the operator ${ }^{(19)} \exp \left[-P_{\perp}(\mathrm{v} \cdot \nabla-L) P_{\perp} t\right]$. The addition of $P_{\perp} \overline{\mathbf{T}}$ takes into account the influence on this propagator of collisions between gas molecules and the sphere during their propagation and it is hard to see how this could prevent the exponential decay. As a consequence, the second term on the right-hand side may be ignored after a few mean free times. In the first term on the right-hand side the exponential acts on a source term which is confined to the surface of the sphere (or at least to the close vicinity thereof; see the next section). As a result the kinetic boundary layer must be confined to a thickness of a few mean free paths around the surface of the sphere.

Furthermore, if we restrict ourselves to the case where $\Psi$ and $\mathbf{V}$ vary only on a hydrodynamic time scale, we may replace $\Psi(t-\tau)$ and $\mathbf{V}(t-\tau)$ by $\Psi(t)$ and $\mathbf{V}(t)$, and we may extend the upper bound of the $t$ integration to $\infty$. Under these approximations, the equation for the boundary layer part of $\Psi$ becomes

$$
\begin{aligned}
P_{\perp} \Psi(\mathbf{r}, \mathbf{v}, t)= & {\left[P_{\perp}(\mathbf{v} \cdot \nabla-L-\mathbf{T}) P_{\perp}\right]^{-1} } \\
& \times P_{\perp}\left\{\overline{\mathbf{T}} P \Psi-\mathbf{T}\left[\beta m \mathbf{v} \cdot \mathbf{V}(t)-\frac{\beta_{w}-\beta}{\beta}\left(\frac{\beta m}{2} v^{2}-\frac{3}{2}\right)\right]\right\}
\end{aligned}
$$

Substitution into (4.18a) yields

$$
P\left(\frac{\partial}{\partial t}+\mathbf{v} \cdot \nabla-L\right) P \Psi=P \tilde{\mathbf{T}} P \Psi-P \tilde{\mathbf{T}}\left[\beta m \mathbf{v} \cdot \mathbf{V}(t)-\frac{\beta_{w}-\beta}{\beta}\left(\frac{\beta m}{2} v^{2}-\frac{3}{2}\right)\right]
$$

where

$$
\begin{aligned}
& \tilde{\mathbf{T}}=\overline{\mathbf{T}}+\overline{\mathbf{T}}\left[P_{\perp}(\mathbf{v} \cdot \nabla-L-\overline{\mathbf{T}}) P_{\perp}\right]^{-1} \overline{\mathbf{T}} \\
& \tilde{\mathbf{T}}=\mathbf{T}+\overline{\mathbf{T}}\left[P_{\perp}(\mathbf{v} \cdot \nabla-L-\overline{\mathbf{T}}) P_{\perp}\right]^{-1} \mathbf{T}
\end{aligned}
$$

We will separate (4.21) into an equation for the outside solution, far from the surface of the sphere, and an equation for the sources that generate this outside solution. Then $P \Psi$, or equivalently the hydrodynamic boundary conditions at the surface of the sphere, can be determined to lowest order in $l / R$. Before doing so, however, we first make a critical examination of the projection operator introduced by (4.10) and (4.13).

\section{THE PROJECTION OPERATOR RECONSIDERED}

The projection operator onto hydrodynamic space as introduced in (4.10) and (4.13) has some unsatisfactory features that are a consequence 
of the cutoff on the $\mathbf{k}$ integral in (4.10). The main problem can be outlined as follows: As discussed in Section 2, the linearized, extended Boltzmann equation (4.4) can be interpreted as an ordinary Boltzmann equation with source terms given by $\overline{\mathbf{T}}^{\prime} \Psi-\mathbf{T}^{\prime}(\beta m \mathbf{v} \cdot \mathbf{V}+\cdots)$ in (4.4). Generally, the physically interesting solution $\Psi$ of the extended Boltzmann equation (4.4) is an exact solution of the ordinary Boltzmann equation outside the sphere. It is identically zero inside the sphere, it has a source term at the boundary of the sphere, and it has no additional sources anywhere else. The boundary source may be considered to generate $\Psi$, and usually it will generate both a hydrodynamic and a nonhydrodynamic contribution. The latter should decay to zero within a distance of a few mean free paths from the boundary; hence far from the sphere the complete solution is of the hydrodynamic form (4.16). Intuitively one would expect that a decomposition of the complete solution into a hydrodynamic and a nonhydrodynamic part would yield the hydrodynamic solution inside the boundary layer simply as the analytic continuation of the hydrodynamic solution [Eq. (4.16)] far from the boundary. Moreover, both the hydrodynamic and the nonhydrodynamic solution inside the sphere ought to vanish.

Now if one looks at what the projection operator defined by (4.10) actually does, it turns out that hardly anything of this intuitive picture is realized. Due to the cutoff on the $\mathbf{k}$ integration, whenever the projection operator acts on a source term it smears out the $\delta$-function $\delta(r-R)$ over a layer of a few mean free paths thick on either side of the surface of the sphere. Similarly, the hydrodynamic solution generated by this projection operator does not jump discontinuously from some nonzero value just outside the sphere to zero just inside the sphere, but it varies smoothly through the layer of sources from the outside hydrodynamic solution to zero far enough inside the sphere. This solution is not the natural analytic continuation of the solution far outside the sphere, nor is it identically zero inside the sphere, nor is it free of sources everywhere except at surface of the sphere. Of course these unphysical features are compensated for in the nonhydrodynamic solution. The latter exactly cancels the hydrodynamic solution inside the sphere; it contains sources exactly canceling the sources of the hydrodynamic solution everywhere except at the surface of the sphere; and it contains a contribution outside the sphere canceling the difference between the hydrodynamic solution and the analytic continuation of the solution far away. However, one cannot avoid the conclusion that in the neighborhood of the surface of the sphere the decomposition into a hydrodynamic and a nonhydrodynamic part as generated by (4.10) is of a very unphysical nature.

There seem to be two ways out of these problems. The first one is to look at the system on a gross scale, that is, on a scale where all distances 
are measured on a length scale much larger than the mean free path. Under these optics the kinetic boundary layer becomes part of the boundary of the sphere and all detailed information about what goes on there is lost. The operator $P \tilde{\overline{\mathbf{T}}} P$ is to be interpreted as a "generalized particle-sphere collision operator" which describes the hydrodynamic source resulting from the hydrodynamic solution at the surface of the sphere. Only a global knowledge of the action of $P \tilde{\widetilde{T}} P$ is needed to calculate the hydrodynamic solution around the sphere and the drag force exerted on it. This program is worked out in Section 7.

Here and in Section 6 we discuss the other option. That is, we consider $\Psi$ to be given by linear combinations of functions which vanish as $r \rightarrow \infty$ and which satisfy the linearized Boltzmann equation everywhere except perhaps at the surface of the sphere, where they may have discontinuities. These functions may be considered as being solutions of the equation

$$
(\partial / \partial t+\mathbf{v} \cdot \nabla-L) h=\delta(r-R) g(\mathbf{r}, \mathbf{v}, t)
$$

for an appropriate function $g(\mathbf{r}, \mathbf{v}, t)$ to be described further on. The righthand side of the equation will be interpreted as the source generating $h$. It seems reasonable to construct $\Psi$ from such solutions, because $\Psi$ is really generated by sources at the surface of the sphere, as we see in Eq. (4.4). Now we will see if it is possible to decompose the set of all functions of this type into two subsets, one of which we can identify as a hydrodynamic set and the other as a nonhydrodynamic set. ${ }^{7}$

If one considers solutions of the Boltzmann equation driven by a boundary source, it is not possible to distinguish hydrodynamic and nonhydrodynamic solutions by their temporal behavior, because each of these solutions follows the time behavior of the source. The two types of solutions can be distinguished, however, by their spatial behavior. For the case of the drag on a sphere discussed here, it is sufficient to consider only sources varying on a hydrodynamic time scale, since the time scale will ultimately be determined by the time on which $\mathbf{V}(t)$ varies. Then the nonhydrodynamic solutions should spread out from the boundary at a speed on the order of the thermal velocity $\left(k_{\mathrm{B}} T / m\right)^{1 / 2}$ and decay exponentially on the timescale of the mean free time. This will manifest itself as an exponential spatial decay on the length scale of the mean free path. The hydrodynamic solutions, on the other hand, should decay only with an inverse power of time as they move

${ }^{7}$ In carrying out the decomposition, we will have to consider solutions to equations of the above type that do not vanish inside the sphere. It may seem odd at first to consider these solutions, since $\Psi$ must be zero inside the sphere. It will turn out to be advantageous to decompose the complete source of $\Psi$ into a number of contributions, each of which may generate an inside solution. Only in the end will the sum of all inside solutions become zero. 
out and this will result in a spatial decay with some inverse power of the distance to the origin.

To obtain the hydrodynamic solutions, we start by Laplace-transforming the linearized Boltzmann equation to

$$
(z+\mathbf{v} \cdot \mathbf{V}-L) \hat{\Psi}=0, \quad r \neq R
$$

where $\hat{\Psi}$ is the Laplace transform of $\Psi(\mathbf{r}, \mathbf{v}, t)$. In (5.1) we neglect the transient solution connected with the initial distribution function. Now Eq. (5.1) can be used to obtain $z$-dependent linearized hydrodynamic equations by means of the Chapman-Enskog procedure as indicated in Appendix A. Then we will define hydrodynamic solutions of Eq. (5.1) to be solutions of the form (4.16) with $\delta n, \delta T$, and $\mathbf{u}$ given by solutions of the linearized $z$ dependent hydrodynamic equations derived in Appendix A.

If one then assumes that the set of solutions for given $z$ [with

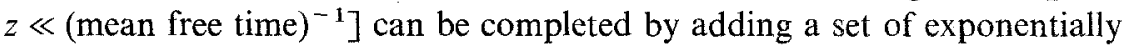
decaying nonhydrodynamic solutions, a unique decomposition of an arbitrary solution into a hydrodynamic and a nonhydrodynamic part ought to be possible. Then the projection operator $P$ onto hydrodynamic space must be defined such that it reduces any solution to its hydrodynamic part. In principle one could construct $P$ in the following way: Suppose that for given $z,\left\{\hat{\Psi}_{\alpha}(\mathbf{r}, \mathbf{v}, z)\right\}$ is a complete set of hydrodynamic and nonhydrodynamic solutions of (5.1). Then one should construct a set of functions $\left\{\hat{\phi}_{\alpha}(\mathbf{r}, \mathbf{v}, z)\right\}$ such that $\left\langle\hat{\phi}_{\alpha}(\mathbf{r}, \mathbf{v}, z) \mid \Psi_{\beta}(\mathbf{r}, \mathbf{v}, z)\right\rangle=\delta_{\alpha \beta}$, with the inner product given by (4.8). The projection operator then would assume the form $P=\sum_{n . s .}\left|\hat{\Psi}_{\alpha}\right\rangle\left\langle\hat{\phi}_{\alpha}\right|$, where the sum over $\alpha$ is restricted to the set of hydrodynamic solutions. In fact, we cannot obtain this explicit form of the projection operator because we do not know the nonhydrodynamic solutions, and hence cannot construct the set $\left\{\hat{\phi}_{\alpha}\right\}{ }^{8}$ Yet there is no problem in determining the action of $P$ on an arbitrary solution of the Boltzmann equation. In the case we were considering we can fruitfully make use of the following tensorial property: if the source occurring in the equation

$$
(\partial / \partial t+\mathbf{v} \cdot \nabla-L-\overline{\mathbf{T}}) \Psi=S \delta(r-R)
$$

${ }^{8}$ Because of the possible discontinuities at the surface of the sphere, and because both the hydrodynamic and nonhydrodynamic solutions are defined for the same $z$, the nonhydrodynamic solutions of the adjoint of Eq. (5.1) are not orthogonal to the hydrodynamic solutions of Eq. (5.1), and vice versa. For the special case of the Kramers problem of the flow of a gas past a plate and in the BGK approximation, both the hydrodynamic and nonhydrodynamic solutions can be constructed explicitly. ${ }^{(6)}$ There it can be seen that the nonhydrodynamic solutions decay exponentially, and that the orthogonality relations are quite intricate. 
is such that $S$ has the form

where

$$
S=\cos (l \Theta) f^{(1)}\left(v_{r}, v_{\Theta}{ }^{2}, v^{2}, r\right)+\sin (l \Theta) f^{(2)}\left(v_{r}, v_{\Theta}{ }^{2}, v^{2}, r\right) v_{\Theta}
$$

$$
\cos \Theta=\hat{\mathbf{z}} \cdot \hat{\mathbf{r}}, \quad v_{\Theta}=\mathbf{v} \cdot \hat{\boldsymbol{\Theta}}, \quad \hat{\boldsymbol{\Theta}}=-\hat{\mathbf{r}} \times(\hat{\mathbf{r}} \times \hat{\mathbf{z}}) / \sin \Theta
$$

and $f^{(1)}$ and $f^{(2)}$ are arbitrary functions of their arguments, then the resulting $\Psi$ is of the same functional form. This can be seen by solving the equation for $\Psi$ in terms of the source $S$ using the operator $(\mathbf{v} \cdot \nabla-L-\overline{\mathbf{T}})^{-1}$. Then if one uses the representation of $\mathbf{v} \cdot \nabla$ in spherical coordinates, as well as the fact that both $L$ and $\overline{\mathbf{T}}$ are invariant under rotation about the origin, the result follows. In the case of (4.4) with $\beta_{w}=\beta$ and the $z$-axis along $\mathbf{v}$, the source term is of this form with $l=1$.

This means that of all the different hydrodynamic solutions that follow from (A.6), (A.7), (A.9), (A.15), (A.16), and (A.19) by substitution of the hydrodynamic fields obtained there into (4.16), only those for which $l=1$ can actually appear in the case of flow around a sphere we are considering here. To be explicit: For a given eigenvalue $z$, the projection operator $P$ projects onto a space spanned by three linearly independent outside distribution functions $\hat{\Phi}_{1}, \hat{\Phi}_{2}, \hat{\Phi}_{3}$ and three linearly independent inside distribution functions $\hat{\Phi}_{4}, \hat{\Phi}_{5}, \hat{\Phi}_{6}$, all of the form (4.16), with hydrodynamic flow fields that are obtained from Appendix $\mathrm{A}$ as

$$
\begin{aligned}
\hat{\Phi}_{1}: \quad \mathbf{u}= & W(r) u_{0}\left\{\left[\frac{R \rho^{2}}{r^{3}}\left(e^{(R-r) / \rho}-1\right)+\frac{R \rho}{r^{2}}\left(e^{(R-r) / \rho}-\frac{R}{r}\right)\right.\right. \\
& \left.+\frac{R}{r}\left(e^{(R-r) / \rho}-\frac{1}{2} \frac{R^{2}}{r^{2}}\right)-\frac{1}{6} \frac{R^{4}}{r^{3} \rho}\right] \hat{\mathbf{z}} \\
& -\left[\frac{3 R \rho^{2}}{r^{3}}\left(e^{(R-r) / \rho}-1\right)+\frac{3 R \rho}{r^{2}}\left(e^{(R-r) / \rho}-\frac{R}{r}\right)\right. \\
& \left.\left.+\frac{R}{r}\left(e^{(R-r) / \rho}-\frac{3}{2} \frac{R^{2}}{r^{2}}\right)-\frac{1}{2} \frac{R^{4}}{r^{3} \rho}\right](\hat{\mathbf{z}} \cdot \hat{\mathbf{r}}) \hat{\mathbf{r}}\right\} \\
\frac{\delta n}{n}= & W(r) \beta m v u_{0} \frac{R}{r^{2}}\left(1+\frac{R}{\rho}+\frac{1}{2} \frac{R^{2}}{\rho^{2}}+\frac{1}{6} \frac{R^{3}}{\rho^{3}}\right) \hat{\mathbf{z}} \cdot \hat{\mathbf{r}} \\
\frac{\delta T}{T}= & 0 \\
\hat{\Phi}_{2}: \quad \mathbf{u}= & W(r) u_{0} \frac{R^{3}}{r^{3}}[\hat{\mathbf{z}}-3(\hat{\mathbf{z}} \cdot \hat{\mathbf{r}}) \hat{\mathbf{r}}] \\
\frac{\delta n}{n}= & -W(r) \beta m v u_{0} \frac{R^{3}}{r^{2} \rho^{2}} \hat{\mathbf{z}} \cdot \hat{\mathbf{r}} \\
\frac{\delta T}{T}= & 0
\end{aligned}
$$


$\hat{\Phi}_{3}: \quad \mathbf{u}=W(r) D_{T} \frac{R^{2}}{r^{2}}\left\{\left[e^{(R-r) / \sigma}\left(\frac{1}{\sigma}+\frac{1}{r}\right)-\frac{1}{r}\right] \hat{\mathbf{z}}\right.$

$$
\left.-\left[e^{(R-r) / \sigma}\left(\frac{r}{\sigma^{2}}+\frac{3}{\sigma}+\frac{3}{r}\right)-\frac{3}{r}\right](\hat{\mathbf{z}} \cdot \hat{\mathbf{r}}) \hat{\mathbf{r}}\right\}
$$

$$
\frac{\delta n}{n}=-\frac{\delta T}{T}=W(r)\left(\frac{R^{2}}{r^{2}}+\frac{R^{2}}{\sigma r}\right) e^{(R-r) / \sigma} \hat{\mathbf{z}} \cdot \hat{\mathbf{r}}
$$

$\hat{\Phi}_{4}: \quad \mathbf{u}=[1-W(r)] u_{0} \hat{\mathbf{z}}$

$$
\begin{aligned}
\frac{\delta n}{n} & =-[1-W(r)] \beta m v u_{0} \frac{r}{\rho^{2}}(\hat{\mathbf{z}} \cdot \hat{\mathbf{r}}) \\
\frac{\delta T}{T} & =0
\end{aligned}
$$

$\hat{\Phi}_{5}: \quad \mathbf{u}=[1-W(r)] u_{0} \frac{\rho}{R} \llbracket\left\{\frac{2}{3} \frac{\rho}{R}+\left(1+\frac{\rho}{R}\right)\right.$

$$
\begin{aligned}
& \left.\times\left[e^{(r-R) / \rho}\left(-\frac{\rho}{2 r}+\frac{\rho^{2}}{2 r^{2}}-\frac{\rho^{3}}{2 r^{3}}\right)+e^{-(r+R) / \rho}\left(\frac{\rho}{2 r}+\frac{\rho^{2}}{2 r^{2}}+\frac{\rho^{3}}{2 r^{3}}\right)\right]\right\} \hat{\mathbf{z}} \\
& +\left(1+\frac{\rho}{R}\right)\left[e^{(r-R) / \rho}\left(\frac{\rho}{2 r}-\frac{3 \rho^{2}}{2 r^{2}}+\frac{3 \rho^{3}}{2 r^{3}}\right)\right. \\
& \left.-e^{-(r+R) / \rho}\left(\frac{\rho}{2 r}+\frac{3 \rho^{2}}{2 r^{2}}+\frac{3 \rho^{3}}{2 r^{3}}\right)\right](\hat{\mathbf{z}} \cdot \hat{\mathbf{r}}) \hat{\mathbf{r}}
\end{aligned}
$$

$$
\frac{\delta n}{n}=-\frac{2}{3}[1-W(r)] \beta m v u_{0} \frac{r}{R^{2}}(\hat{\mathbf{z}} \cdot \hat{\mathbf{r}})
$$$$
\frac{\delta T}{T}=0
$$

$\hat{\Phi}_{6}: \quad \mathbf{u}=-\frac{3}{4}[1-W(r)] \frac{D_{T}}{R}\left\{\left[e^{(r-R) / \sigma}\left(\frac{\sigma^{2}}{r^{2}}-\frac{\sigma^{3}}{r^{3}}\right)+e^{-(r+R) / \sigma}\right.\right.$

$$
\begin{aligned}
& \left.\times\left(\frac{\sigma^{2}}{r^{2}}+\frac{\sigma^{3}}{r^{3}}\right)-\frac{2}{3}\right] \hat{\mathbf{z}}+\left[e^{(r-R) / \sigma}\left(\frac{\sigma}{r}-\frac{3 \sigma^{2}}{r^{2}}+\frac{3 \sigma^{3}}{r^{3}}\right)\right. \\
& \left.-e^{-(r+R) / \sigma}\left(\frac{\sigma}{r}+\frac{3 \sigma^{2}}{r^{2}}+\frac{3 \sigma^{3}}{r^{3}}\right)\right](\hat{\mathbf{z}} \cdot \hat{\mathbf{r}}) \hat{\mathbf{r}}
\end{aligned}
$$

$$
\begin{aligned}
\frac{\delta n}{n}= & -\frac{\delta T}{T}=-\frac{3}{4}[1-W(r)] \frac{\sigma^{2}}{r R} \\
& \times\left[e^{(r-R) / \sigma}\left(1-\frac{\sigma}{r}\right)+e^{-(r+R) / \sigma}\left(1+\frac{\sigma}{r}\right)\right](\hat{\mathbf{z}} \cdot \hat{\mathbf{r}})
\end{aligned}
$$


Here $u_{0}$ is an arbitrary velocity; $\rho=(v / z)^{1 / 2}$ and $\sigma=\left(D_{T} / z\right)^{1 / 2} ; z$ is the Laplace variable; and $\delta n$ and $\delta T$ are deviations of the density and temperature from their equilibrium values. Furthermore, we have assumed that the typical timescale $t_{H}$ for variations of fluid fields is of the order $t_{H} \sim R^{2} / v$, where $R^{-1}$ is the characteristic strength of a gradient. In this approximation transient sound mode phenomena may be neglected. For a discussion of Brownian motion in the case where the latter are also included see Ref. 20.

Generally, we may say that in the system we are considering, the hydrodynamic part of the Laplace-transformed distribution function has to be of the form

$$
P \hat{\Psi}=\sum_{i=1}^{6} \lambda_{i} \hat{\Phi}_{i}
$$

In the particular case of interest to us here, the coefficients $\lambda_{i}$ can be determined from the strengths of the currents of momentum, energy, and particles in the radial direction. These currents are defined microscopically as

$$
\begin{array}{ll}
J_{1}=m(\mathbf{v} \cdot \hat{\mathbf{r}})^{2}, & J_{2}=m(\mathbf{v} \cdot \hat{\boldsymbol{\Theta}})(\mathbf{v} \cdot \hat{\mathbf{r}}) \\
J_{3}=\frac{1}{2} m v^{2}(\mathbf{v} \cdot \hat{\mathbf{r}}), & J_{4}=\mathbf{v} \cdot \hat{\mathbf{r}}
\end{array}
$$

and their strengths when the gas is described by a distribution function $\hat{\Psi}$ are given as ${ }^{9}$

$$
j_{i}(\mathbf{r})=\left\langle J_{i}(\mathbf{v}) \hat{\Psi}(\mathbf{v}, \mathbf{r})\right\rangle
$$

The expectation value $\langle\cdots\rangle$ is defined by $\langle f(\mathbf{v})\rangle=n \int d \mathbf{v} \varphi(v) f(\mathbf{v})$.

As we show in Appendix B, both outside and inside the boundary layer the radial currents are determined, at least to leading order in the Knudsen number $l / R$, by the hydrodynamic part of the distribution function alone. Then we may write that

$$
\left\langle J_{k} \hat{\Psi}\right\rangle=\left\langle J_{k} P \hat{\Psi}\right\rangle=\sum_{i=1}^{6} \lambda_{i}\left\langle J_{k} \hat{\Phi}_{i}\right\rangle
$$

Apparently we have four equations here with six unknown constants $\lambda_{i}$, but in fact these equations fall into two independent sets of equations because $\hat{\Phi}_{1}, \hat{\Phi}_{2}$, and $\hat{\Phi}_{3}$ are nonzero outside the sphere only and $\hat{\Phi}_{4}, \hat{\Phi}_{5}$, and $\hat{\Phi}_{6}$ inside the sphere only. These equations are

$$
\begin{array}{ll}
\sum_{i=1}^{3} \lambda_{i}\left\langle J_{k} \hat{\Phi}_{i}\right\rangle=\left\langle J_{k} \hat{\Psi}\right\rangle & r>R \\
\sum_{i=4}^{6} \lambda_{i}\left\langle J_{k} \hat{\Phi}_{i}\right\rangle=\left\langle J_{k} \hat{\Psi}\right\rangle & r<R
\end{array}
$$

\footnotetext{
${ }^{9}$ Due to the rotational symmetry, the strength of the current $J_{s}=m(\mathbf{v} \cdot \hat{\varphi})(\mathbf{v} \cdot \hat{\mathbf{r}})$ is always zero.
} Here $\hat{r}, \hat{\boldsymbol{\Theta}}, \hat{\varphi}$ form a set of orthogonal unit vectors. 
Either set of four equations contains only three unknowns. However, since $P \hat{\Psi}$ has to be of the form (5.4), one of the four equations in each set must be implied by the other three. As we shall see, it is advantageous that even a determination of the $j_{i}$ inside the boundary layer can be used to determine the $\lambda_{i}$.

Having answered the question of how to determine the action of the hydrodynamic projection operator on an arbitrary distribution function, we are faced with the harder problem of how to determine its action on a source $S$. This problem can be attacked as follows. As discussed in Section 2 for the nonlinear equation, the source of an outside solution of the equation

$$
(z+\mathbf{v} \cdot \nabla-L) \hat{\Psi}=S
$$

has to satisfy the identity $S=\hat{\Psi}(\mathbf{v} \cdot \hat{\mathbf{r}}) \delta(r-R)$. Similarly the source of an inside solution has to satisfy $S=-\hat{\Psi}(\mathbf{v} \cdot \hat{\mathbf{r}}) \delta(r-R)$. Hence a source of a general solution of the form (4.2) must satisfy

$$
S=\left(\hat{\Psi}^{\mathrm{ext}}-\hat{\Psi}^{\mathrm{int}}\right)(\mathbf{v} \cdot \hat{\mathbf{r}}) \delta(r-R)
$$

"Dividing by" $(\mathbf{v} \cdot \hat{\mathbf{r}}) \delta(r-R)$, one obtains

$$
\hat{\Psi}^{\mathrm{ext}}-\hat{\Psi}^{\mathrm{int}}=\frac{1}{(\mathbf{v} \cdot \hat{\mathbf{r}}) \delta(r-R)} S, \quad r=R
$$

This equation reformulates (5.9) as a boundary value problem: What is the solution of the linearized Boltzmann equation which decays to zero at infinity and has a discontinuity at the surface of the sphere given by the right-hand side of (5.11)? Here immediately questions of existence and uniqueness occur. We do not know very much about the problem of the existence of a solution of (5.11) for an arbitrary given $S$. If one picks a $\hat{\Psi}^{\text {ext }}$ and $\hat{\Psi}^{\text {int }}$ at the boundary satisfying (5.11), one can in principle construct $\hat{\Psi}$ everywhere by integration of the linearized Boltzmann equation along characteristics (lines parallel to v), but there is no guarantee whatever that the solution thus obtained will decay to zero at infinity. Of course, given only $\hat{\Psi}^{\text {ext }}-\hat{\Psi}^{\text {int }}$, there are infinitely many ways to pick $\hat{\Psi}^{\text {ext }}$ and $\hat{\Psi}^{\text {int }}$, but whether there is always at least one way which leads to a solution satisfying the boundary conditions at infinity is impossible to tell. However, the sources we have to deal with are always of a physical nature (of the type $\overline{\mathbf{T}} \hat{\mathbf{T}}$ ), and for that reason we will assume they will not cause mathematical difficulties of the type sketched above. More can be said about the question of uniqueness. Suppose $\hat{\Psi}$ and $\hat{\Psi}+\Delta \hat{\Psi}$ both satisfy (5.9) for a given source $S$. Then $\Delta \hat{\Psi}$ is an eigensolution of the homogeneous linearized Boltzmann equation with eigenvalue $z$. Any solution of the linearized Boltzmann equation which is not driven by any sources will eventually decay to zero. Hence if any such solutions are present initially they will die off after a sufficiently long time. 
In (5.9) these transient solutions would be generated by the initial value term resulting from the Laplace transform of the Boltzmann equation. But we have omitted this initial value term just because we were not interested in the transient phenomena, but only in the distribution function generated by the motion of the sphere. Therefore we suppose that the solution of (5.9), if it exists, is unique.

After this disgression on the mathematical properties of (5.9), let us return to the question of how to determine $P S$. Assume first that for $S$ the solution $\hat{\Psi}$ of (5.9) can be constructed satisfying formally

$$
\hat{\Psi}=(z+\mathbf{v} \cdot \nabla-L)^{-1} S
$$

Then $\hat{\Psi}$ can be decomposed into $\hat{\Psi}=P \hat{\Psi}+(1-P) \hat{\Psi}$ and, with the aid of (5.10), $S$ can be decomposed accordingly as

$$
\begin{aligned}
S= & (\mathbf{v} \cdot \hat{\mathbf{r}}) \delta(r-R) P\left\{\hat{\Psi}^{\mathrm{ext}}-\hat{\Psi}^{\mathrm{int}}\right\} \\
& +(\mathbf{v} \cdot \hat{\mathbf{r}}) \delta(r-R)(1-P)\left\{\hat{\Psi}^{\mathrm{ext}}-\hat{\Psi}^{\mathrm{int}}\right\}
\end{aligned}
$$

The source term $(\mathbf{v} \cdot \hat{\mathbf{r}}) \delta(r-R) P\left\{\hat{\Psi}^{\mathrm{ext}}-\hat{\Psi}^{\text {int }}\right\}$ is the source of a purely hydrodynamic solution $P \hat{\Psi}$. To see this, one needs only to consider Eq. (5.10) and realize that $P \hat{\Psi}$ is by itself a solution of the linearized Boltzmann equation. Similarly, $(\mathbf{v} \cdot \hat{\mathbf{r}}) \delta(r-R)(1-P)\left\{\hat{\Psi}^{\text {ext }}-\hat{\Psi}^{\text {int }}\right\}$ is the source of purely nonhydrodynamic solution.

The source $(\mathbf{v} \cdot \hat{\mathbf{r}}) \delta(r-R) P\left\{\hat{\Psi}^{\text {ext }}-\hat{\Psi}^{\text {int }}\right\}$ is defined to be the projection of $S$ onto hydrodynamic space. Combining (5.12) and (5.13), one can write $P S$ formally as

$$
\begin{aligned}
P S= & (\mathbf{v} \cdot \hat{\mathbf{r}}) \delta(r-R) P\left\{\hat{\Psi}^{\mathrm{ext}}-\hat{\Psi}^{\mathrm{int}}\right\} \\
= & (\mathbf{v} \cdot \hat{\mathbf{r}}) \delta(r-R) \\
& \times P\left\{\left[(z+\mathrm{v} \cdot \nabla-L)^{-1} S\right]^{\mathrm{ext}}-\left[(z+\mathbf{v} \cdot \nabla-L)^{-1} S\right]^{\mathrm{int}}\right\}
\end{aligned}
$$

The formal prescription given above for the construction of $P S$ given $S$ cannot be followed as such in practice, because in general the solution of (5.12) (construct $\hat{\Psi}$, given $S$ ) is not known. However, although the complete solution is not known, its hydrodynamic part can be obtained, at least to leading order in $l / R$, by using the property of the radial currents given in Eq. (5.8).

Given $S$, one can use Eq. (5.11) to determine $\hat{\Psi}^{\text {ext }}-\hat{\Psi}^{\text {int }}$, the discontinuity of $\hat{\Psi}$ at the surface of the sphere. Then Eq. (5.6) can be used to compute $j_{i}^{\text {ext }}-j_{i}^{\text {int }}$, the discontinuity of the radial currents at the surface of the sphere. Next, since to leading order in $l / R$ the radial currents are deter- 
mined by the hydrodynamic part of the distribution function, even inside the boundary layer, we can write

$$
j_{i}^{\mathrm{ext}}-j_{i}^{\mathrm{int}}=\left\langle J_{i} P\left\{\hat{\Psi}^{\mathrm{ext}}-\hat{\Psi}^{\mathrm{int}}\right\}\right\rangle=\sum_{j=1}^{3} \lambda_{j}\left\langle J_{i} \hat{\Phi}_{j}\right\rangle-\sum_{j=4}^{6} \lambda_{j}\left\langle J_{i} \hat{\Phi}_{j}\right\rangle
$$

Hence the values of $j_{i}^{\text {ext }}-j_{i}^{\text {int }}$ obtained from a given source $S$ can be used to find the coefficients $\lambda_{j}$ if one expresses $P \hat{\Psi}$ generated by $P S$ by the way of (5.4). The hydrodynamic part of $S$ then becomes

$$
P S=(\mathbf{v} \cdot \hat{\mathbf{r}}) \delta(r-R)\left(\sum_{i=1}^{3} \lambda_{i} \hat{\Phi}_{i}-\sum_{i=4}^{6} \lambda_{i} \hat{\Phi}_{i}\right)
$$

in accordance with (5.14).

One problem does remain here. Since there are only four different radial currents and there are six independent hydrodynamic distribution functions $\hat{\Phi}_{i}$ (just at the surface both inside and outside solutions come into play), the coefficients cannot be determined uniquely. In the case of the flow around a sphere which we are considering, this problem can be overcome by using the fact that in the end the distribution function inside the sphere has to vanish. ${ }^{10}$

The details of all these calculations, as well as a discussion how to deal with the nonuniqueness of the $\lambda_{i}$, are given in Section 6 .

\section{SOLUTION OF THE EXTENDED BOLTZMANN EQUATION}

Having redefined the projection operator, we are in a position now to explicitly solve the extended Boltzmann equation to leading order in $l / R$.

We start by Laplace-transforming (4.21),

$$
P(z+\mathbf{v} \cdot \nabla-L) P \hat{\Psi}=P \tilde{\mathbf{T}} P \hat{\Psi}-P \tilde{\mathbf{T}} \beta m[\mathbf{v} \cdot \mathbf{V}(z)]
$$

where we have set $\beta_{w}=\beta$, dropped the initial condition term $P \Psi(0)$, and where $P$ is now the projection operator defined in Section 5 . It is worth mentioning that $(z+\mathbf{v} \cdot \boldsymbol{\nabla}-L) P \hat{\Psi}$ is equal to zero everywhere except at the surface of the sphere, so that the projection operator acting on this combination from the left is the type of projection which acts on a source.

${ }^{10}$ One might ask if $\lambda_{4}, \lambda_{5}, \lambda_{6}$ cannot be set equal to zero immediately, since we expect that $P \hat{\Psi}$ as well as $\hat{\Psi}$ vanishes inside the sphere. However, an arbitrary source $S$ will in general produce a nonzero inside solution; hence if the source of $P \hat{\Psi}$ is considered as consisting of different contributions, each of these may produce an inside solution and only in the end will the sum of all inside solutions cancel. 
Equation (6.1) can be rewritten as

$$
\begin{aligned}
P \hat{\Psi} & -\{P(z+\mathbf{v} \cdot \nabla-L) P\}^{-1} P \tilde{\mathbf{T}} P \hat{\Psi} \\
& =-\{P(z+\mathbf{v} \cdot \mathbf{\nabla}-L) P\}^{-1} \tilde{\mathbf{T}} \beta m \mathbf{v} \cdot \mathbf{V}(z)
\end{aligned}
$$

where the inverse operator $\{P(z+\mathbf{v} \cdot \nabla-L) P\}^{-1}$ is such that when it acts on a hydrodynamic source $P S$ it produces the hydrodynamic solution for which $P S$ is the source.

Let us first investigate the structure of the terms on the left-hand side of this equation: $P \hat{\Psi}$ must be of the form (5.4). We will find it convenient to represent $P \hat{\Psi}$ by a six-component vector with components $\lambda_{i}$. In our notation we will use the symbol $P \hat{\Psi}$ to denote this vector as well as the distribution function (5.4). The operator $P \tilde{\overline{\mathbf{T}}} P$ transforms any hydrodynamic distribution function into the source of some other hydrodynamic distribution function. If it acts upon a distribution function of the form (5.4), $\sum \lambda_{i} \hat{\Phi}_{i}$, it generates a source of the form (5.16),

$$
(\mathbf{v} \cdot \hat{\mathbf{r}}) \delta(r-R)\left(\sum_{i=1}^{3} \gamma_{i} \hat{\Phi}_{i}-\sum_{i=4}^{6} \gamma_{i} \hat{\Phi}_{i}\right)
$$

for some set $\left\{\gamma_{i}\right\}$. There are six linearly independent distribution functions of the form (5.4). There are also six linearly independent sources of the form (5.16). If one chooses a basis $\left(S_{1}, S_{2}, \ldots, S_{6}\right)$ for these sources, it follows that $P \tilde{\overline{\mathbf{T}}} P$ can be represented by a $6 \times 6$ matrix, the components of which describe the amount of $S_{i}$ generated by $P \tilde{\overline{\mathrm{T}}} P$ acting on $\hat{\Phi}_{j}$.

How should the basic sources $S_{1}, \ldots, S_{6}$ be chosen? The choice suggested by (5.16), to take the sources $(\mathbf{v} \cdot \hat{\mathbf{r}}) \delta(r-R) \hat{\Phi}_{i}$, is not the most practical one. As discussed in the preceding section, the action of the projection operator $P$ on an arbitrary source $S$ is to be determined from the discontinuities in the radial currents, given by

$$
\delta(r-R)\left(j_{i}^{\mathrm{ext}}-j_{i}^{\mathrm{int}}\right)=\left\langle J_{i}[1 /(\mathrm{v} \cdot \hat{\mathbf{r}})] S\right\rangle=\left\langle n_{i} S\right\rangle
$$

Moreover, it follows from Eqs. (5.7) and (5.11) that

$$
\begin{aligned}
\delta(r-R)\left(j_{i}^{\mathrm{ext}}-j_{i}^{\mathrm{int}}\right) & =\delta(r-\mathrm{R})\left\langle J_{i} P\left(\hat{\Psi}^{\mathrm{ext}}-\hat{\Psi}^{\mathrm{int}}\right)\right\rangle \\
& =\left\langle J_{i}[1 /(\mathbf{v} \cdot \hat{\mathbf{r}})] P S\right\rangle=\left\langle n_{i} P S\right\rangle
\end{aligned}
$$

where the densities $n_{i}$ are defined as

$$
n_{1}=m \mathbf{v} \cdot \hat{\mathbf{r}}, \quad n_{2}=m \mathbf{v} \cdot \hat{\boldsymbol{\Theta}}, \quad n_{3}=m v^{2} / 2, \quad n_{4}=1
$$

The hydrodynamic distribution function generated by $P S$ is a distribution function of the form (5.4) that exhibits precisely the discontinuities in the radial currents following from (6.3). PS itself is obtained from (5.14). Now, 
as mentioned in Section 5, one is confronted with the problem that the discontinuities in the radial currents do not uniquely determine the hydrodynamic part of the distribution function generated by $S$. The reason is that there are six independent functions $\Phi_{i}$ and only four independent radial currents. The resolution of this difficulty is simplified by passing to a different basis for the set of hydrodynamic distribution functions,

$$
\begin{aligned}
& \hat{\Psi}_{1}=\hat{\Phi}_{1}+\left(1+\frac{1}{3} R / \rho\right) \hat{\Phi}_{4} \\
& \hat{\Psi}_{2}=\hat{\Phi}_{2}-2 \hat{\Phi}_{4} \\
& \hat{\Psi}_{3}=\hat{\Phi}_{3}-D_{T}\left(2 / \sigma+R / \sigma^{2}\right) \hat{\Phi}_{4} \\
& \hat{\Psi}_{4}=\hat{\Phi}_{4} \\
& \hat{\Psi}_{5}=\hat{\Phi}_{5}+c_{1} \hat{\Psi}_{1}+c_{2} \hat{\Psi}_{2}+c_{3} \hat{\Psi}_{4} \\
& \hat{\Psi}_{6}=\hat{\Phi}_{6}+c_{4} \hat{\Psi}_{1}+c_{5} \hat{\Psi}_{5}+c_{6} \hat{\Psi}_{3}+c_{7} \hat{\Psi}_{4}
\end{aligned}
$$

with coefficients $c_{1}, \ldots, c_{7}$ given by

$$
\begin{aligned}
c_{1}= & -\frac{5}{12} \frac{R}{\rho}+\frac{5}{6}-\frac{5}{4} \frac{\rho}{R}+\frac{5}{2} \frac{\rho^{3}}{R^{3}}+e^{-2 R / \rho}\left(-\frac{5}{12} \frac{R}{\rho}-\frac{5}{3}-\frac{15}{4} \frac{\rho}{R}-\frac{5 \rho^{2}}{R^{2}}-\frac{5}{2} \frac{\rho^{3}}{R^{3}}\right) \\
c_{2}= & -\frac{1}{6} \frac{\rho}{R}\left(1+\frac{\rho}{R}\right)\left[-\frac{1}{2}+\frac{3}{2} \frac{\rho}{R}-\frac{3 \rho^{2}}{R^{2}}+\frac{3 \rho^{3}}{R^{3}}\right. \\
& \left.+e^{-2 R / \rho}\left(-\frac{1}{2}-\frac{3}{2} \frac{\rho}{R}-\frac{3 \rho^{2}}{R^{2}}-\frac{3 \rho^{3}}{R^{3}}\right)\right] \\
c_{3}= & -\frac{\rho}{R}\left\{\frac{2}{3} \frac{\rho}{R}+\left(1+\frac{\rho}{R}\right)\left[-\frac{\rho^{2}}{R^{2}}+\frac{\rho^{3}}{R^{3}}+e^{-2 R / \rho}\left(-\frac{\rho^{2}}{R^{2}}-\frac{\rho^{3}}{R^{3}}\right)\right]\right\} \\
c_{4}= & -3 z R\left\{-1+\frac{1}{4} \frac{R \sigma}{\rho^{2}}\left[\frac{2 \sigma}{R}-\frac{3 \sigma^{2}}{R^{2}}+\frac{6 \sigma^{3}}{R^{3}}-\frac{6 \sigma^{4}}{R^{4}}\right.\right. \\
& \left.\left.+e^{-2 R / \sigma}\left(1+\frac{2 \sigma}{R}+\frac{2 \sigma^{2}}{R^{2}}\right) \frac{3 \sigma^{2}}{R^{2}}\right]\right\} \\
& \times\left[u_{0}\left(3+\frac{3 R}{\rho}+\frac{3}{2} \frac{R^{2}}{\rho^{2}}+\frac{1}{2} \frac{R^{3}}{\rho^{3}}\right)\left(2+\frac{2 R}{\sigma}+\frac{R^{2}}{\sigma^{2}}\right)\right]^{-1} \\
c_{5}= & -z \sigma\left[\frac{2 \sigma}{R}-\frac{3 \sigma^{2}}{R^{2}}+\frac{6 \sigma^{3}}{R^{3}}-\frac{6 \sigma^{4}}{R^{4}}+e^{-2 R / \sigma}\left(1+\frac{2 \sigma}{R}+\frac{2 \sigma^{2}}{R^{2}}\right) \frac{3 \sigma^{2}}{R^{2}}\right] \\
& \times\left[4 u_{0}\left(2+\frac{2 R}{\sigma}+\frac{R^{2}}{\sigma^{2}}\right)\right]^{-1}
\end{aligned}
$$




$$
\begin{aligned}
& c_{6}=\frac{3 \sigma}{4} \frac{\sigma}{R}\left[1-\frac{2 \sigma}{R}+\frac{2 \sigma^{2}}{R^{2}}+e^{-2 R / \sigma}\left(-1-\frac{2 \sigma}{R}-\frac{2 \sigma^{2}}{R^{2}}\right)\right]\left(2+\frac{2 R}{\sigma}+\frac{R^{2}}{\sigma^{2}}\right)^{-1} \\
& c_{7}=\frac{3}{2} \frac{z \sigma}{u_{0} c_{2}}\left[-\frac{1}{3} \frac{R}{\sigma}-\frac{2}{3}-\frac{2}{3} \frac{\sigma}{R}-\frac{2 \sigma^{3}}{R^{3}}+\frac{2 \sigma^{4}}{R^{4}}-e^{-2 R / \sigma}\left(1+\frac{2 \sigma}{R}+\frac{2 \sigma^{2}}{R^{2}}\right) \frac{\sigma^{2}}{R^{2}}\right]
\end{aligned}
$$

The $\hat{\Psi}_{i}$ differ from the $\hat{\Phi}_{i}$ in two respects: First, an amount $\hat{\Phi}_{4}$ has been subtracted from $\hat{\Phi}_{1}, \hat{\Phi}_{2}, \hat{\Phi}_{3}$ with the aim of obtaining a vanishing discontinuity $j_{4}^{\text {ext }}-j_{4}^{\text {int }}$ for $\hat{\Psi}_{1}, \hat{\Psi}_{2}$, and $\hat{\Psi}_{3}$. This simplifies some of the calculations. Second, $\hat{\Psi}_{5}$ and $\hat{\Psi}_{6}$ have been defined in such a way that the discontinuities $j_{i}^{\text {ext }}-j_{i}^{\mathrm{int}}$ in all four radial currents vanish. It is to obtain this goal that one has to choose the coefficients $c_{1}, \ldots, c_{7}$ in such a complicated way. Suppose now one wants to use the values of the discontinuities in the radial currents given by Eqs. (6.3) to determine the distribution function generated by a hydrodynamic source. The distribution function must have the form

$$
\hat{\Psi}=\sum_{i=1}^{6} \lambda_{i} \hat{\Psi}_{i}
$$

while the hydrodynamic source must have the form

$$
S=\sum_{i=1}^{6} \lambda_{i}(\mathbf{v} \cdot \hat{\mathbf{r}}) \delta(r-R)\left(\hat{\Psi}_{i}^{\mathrm{ext}}-\hat{\Psi}_{i}^{\mathrm{int}}\right)
$$

Then the coefficients $\lambda_{1}, \ldots, \lambda_{4}$ are completely determined by the discontinuities in the radial currents, but the coefficients $\lambda_{5}$ and $\lambda_{6}$ are completely undetermined. In other words, from the coefficients given in (6.3) we can always find how much of the distribution functions $\hat{\Psi}_{1}, \ldots, \hat{\Psi}_{4}$ is generated by a given source $S$, but how much $\hat{\Psi}_{5}$ and $\hat{\Psi}_{6}$ is generated remains completely unknown.

Because of this distinction between $\hat{\Psi}_{1}, \ldots, \hat{\Psi}_{4}$ on the one hand and $\hat{\Psi}_{5}$ and $\hat{\Psi}_{6}$ on the other hand we decided to choose our basic hydrodynamic sources in the following way: $S_{1}, \ldots, S_{6}$ are sources satisfying

$$
\begin{aligned}
\left\langle n_{i} S_{j}\right\rangle=\delta(r-R) \delta_{i j} f_{j}(\Theta), & i=1, \ldots, 4 \\
\{P(z+\mathbf{v} \cdot \nabla-L) P\}^{-1} S_{i}=\sum_{j=1}^{4} \hat{\Psi}_{j} B_{j i}, & i=1, \ldots, 4 \\
S_{i}=(\mathbf{v} \cdot \hat{\mathbf{r}}) \delta(r-R)\left\{\hat{\Psi}_{i}^{\text {ext }}-\hat{\Psi}_{i}^{\text {int }}\right\}, & i=5,6
\end{aligned}
$$

In (6.8), $f_{j}(\Theta)$ is defined as $f_{1}(\Theta)=f_{3}(\Theta)=f_{4}(\Theta)=\cos \Theta$ and $f_{2}(\Theta)=\sin \Theta$. The appearance of these factors in (6.8) is a consequence of (5.2) with $l=1$ and of (6.4). The meaning of (6.9) is that $S_{1}, \ldots, S_{4}$ have been chosen such that they generate distribution functions which are linear combinations 
of $\hat{\Psi}_{1}, \ldots, \hat{\Psi}_{4}$ exclusively and contain no components along $\hat{\Psi}_{5}$ and $\hat{\Psi}_{6}$. As yet the coefficients $B_{j i}$ are unknown. From (5.11)-(5.13) and (6.10) one obtains

$$
(z+\mathbf{v} \cdot \mathbf{\nabla}-L)^{-1} S_{i}=[P(z+\mathbf{v} \cdot \nabla-L) P]^{-1} S_{i}=\hat{\Psi}_{i}, \quad i=5,6
$$

in other words, $S_{5}$ and $S_{6}$ have been chosen to be the exact sources of $\hat{\Psi}_{5}$ and $\hat{\Psi}_{6}$. From (6.8) and the fact that to leading order in $l / R$ the radial currents are determined by the hydrodynamic solutions and that $P S=$ $\sum_{i=1}^{6} \gamma_{i} S_{i}$, it follows that the hydrodynamic projection of a given source $S$ is of the form

$$
P S=\sum_{i=1}^{4} \frac{1}{\delta(r-R) f_{i}(\Theta)}\left\langle n_{i} S\right\rangle S_{i}+\sum_{j=5,6} \gamma_{j} S_{j}
$$

where $\gamma_{5}$ and $\gamma_{6}$ are undetermined constants.

With the aid of (6.12) one can formulate the matrix representation of $P \tilde{\overline{\mathbf{T}}} P$. Let $A_{i j}$ denote the amount of $S_{i}$ generated by $P \tilde{\overline{\mathbf{T}}} P$ acting on $\hat{\Psi}_{j}$. Then, using (6.3b) and (6.8) we find for $i \leqslant 4$ that

$$
\delta(r-R) f_{i}(\Theta) A_{i j}=\left\langle n_{i} \tilde{\mathbf{T}} \hat{\Psi}_{j}\right\rangle, \quad i=1, \ldots, 4
$$

whereas $A_{i j}$ cannot be obtained by our methods for $i=5,6$. A complete calculation of the matrix elements $\left\langle n_{i} \tilde{\overline{\mathbf{T}}} \hat{\Psi}_{j}\right\rangle$ for a given particle-sphere collision operator $\tilde{\overline{\mathbf{T}}}$ is usually not possible, due to our lack of knowledge about what goes on inside the kinetic boundary layer. However, the general form of these matrix elements as a function of the hydrodynamic flow fields occurring in $\hat{\Psi}_{j}$ can be obtained fairly easily. In Appendix $C$ we have worked out matrix elements between the densities $n_{i}$ defined in (6.4) and $\tilde{\overline{\mathbf{T}}}$ acting on an arbitrary hydrodynamic solution $\Psi_{\mathrm{NS}}$ of the form (4.16). For $\tilde{\overline{\mathbf{T}}}$ we considered the cases that $\overline{\mathbf{T}}$ is either the diffuse reflection operator or the operator $\overline{\mathbf{T}}_{\alpha}$ defined in (2.11) corresponding to the Maxwell reflection model. The results are of the general form

$$
\begin{aligned}
& \left\langle n_{1} \tilde{\mathbf{T}} \hat{\Psi}_{\mathrm{NS}}\right\rangle=-n \delta(r-R)\left[\lambda_{1}\left(\mathbf{u}^{(+)} \cdot \hat{\mathbf{r}}\right)+\lambda_{2} \delta T^{(+)} / T\right] \\
& \left\langle n_{2} \tilde{\widetilde{\mathbf{T}}} \hat{\Psi}_{\mathrm{NS}}\right\rangle=-n \delta(r-R) \lambda_{3}\left(\mathbf{u}^{(+)} \cdot \hat{\mathbf{\Theta}}\right) \\
& \left\langle n_{3} \tilde{\mathbf{T}} \hat{\Psi}_{\mathrm{NS}}\right\rangle=n \delta(r-R)\left[\lambda_{4}\left(\mathbf{u}^{(+)} \cdot \hat{\mathbf{r}}\right)-\lambda_{5} \delta T^{(+)} / T\right] \\
& \left\langle n_{4} \tilde{\mathrm{T}} \hat{\Psi}_{\mathrm{NS}}\right\rangle=0
\end{aligned}
$$

where the superscript $(+)$ denotes that for the functions behind the $\delta$-function one has to take the limit as the surface of the sphere is approached from the outside. In the case of diffuse reflection $\lambda_{1}, \ldots, \lambda_{5}$ are unknown constants depending on the structure of the boundary layer, and, to leading order, 
independent of the Knudsen number $l / R$. In the case of the Maxwell reflection mechanism one has, for $\alpha$ of order $l / R$,

$$
\lambda_{3}(\alpha)=-\alpha(2 \pi \beta / m)^{-1 / 2}
$$

Furthermore, $\lambda_{2}, \lambda_{4}$, and $\lambda_{5}$ are also proportional to $\alpha$ in that case, but $\lambda_{1}$ remains independent of this parameter. The matrix elements $A_{i j}$ with $i \leqslant 4$ can be obtained simply from (6.14) by substituting for the flow fields the values occurring in $\hat{\Psi}_{j}$. However, we postpone this calculation. We still remark that in spite of the occurrence of several unknown constants in (6.14) we will be able to calculate the distribution function $\hat{\Psi}$ around the sphere to leading order in $l / R$.

For a further evaluation of the second term on the left-hand side of (6.2) we also need to know the action of the operator $[P(z+v \cdot \nabla-L) P]^{-1}$. This operator acts on a hydrodynamic source of the form

$$
S=\sum_{i=1}^{6} \gamma_{i} S_{i}
$$

and it generates a hydrodynamic distribution function of the form (6.6). Hence it can be represented, like the operator $P \tilde{\widetilde{T}} P$, by a $6 \times 6$ matrix. This matrix will be called B. It is defined such that $B_{i j}$ is the amount of $\hat{\Psi}_{i}$ generated by the source $S_{j}$ or

$$
[P(z+\mathbf{v} \cdot \mathbf{V}-L) P]^{-1} S_{i}=\sum_{j=1}^{6} \hat{\Psi}_{j} B_{j i}
$$

The notation used in (6.9) anticipated this definition. From the discussion below Eq. (6.10) it follows that $B_{j i}=0$ for $j>4$ and $i \leqslant 4$. From (6.11) it follows furthermore that $B_{i j}=\delta_{i j}$ for $j>4$. Hence $\mathbf{B}$ factorizes into a $4 \times 4$ matrix $\mathbf{B}^{*}$ with $i, j \leqslant 4$ and two $1 \times 1$ unit matrices $B_{55}$ and $B_{66}$. The elements of $B^{*}$ are obtained by multiplying (6.9) by $P(z+\mathbf{v} \cdot \nabla-L) P$, yielding

$$
\begin{aligned}
S_{i} & =\sum_{j=1}^{4} P(z+\mathbf{v} \cdot \nabla-L) P \hat{\Psi}_{j} B_{j i}^{*} \\
& =\sum_{j=1}^{4}(\mathbf{v} \cdot \hat{\mathbf{r}}) \delta(r-R)\left\{\hat{\Psi}_{j}^{\mathrm{ext}}-\hat{\Psi}_{j}^{\mathrm{int}}\right\} B_{j i}^{*}
\end{aligned}
$$

where we used again that $(z+\mathbf{v} \cdot \nabla-L)$ acting on $\hat{\Psi}_{i}$ produces zero everywhere except at the surface of the sphere, where it yields the source obtained in $(6.18 \mathrm{~b})$. Next, taking the inner products of $(6.18 \mathrm{~b})$ with the densities $n_{k}$ leads to

$$
f_{k}(\Theta) \delta_{k i}=\sum_{j=1}^{4}\left\langle J_{k}\left(\hat{\Psi}_{j}^{\text {ext }}-\hat{\Psi}_{j}^{\text {int }}\right)\right\rangle B_{j i}^{*}
$$

where (6.8) has been used. 
Hence $\mathbf{B}^{*}$ is the inverse of the matrix given by

$$
f_{k}(\Theta)\left[\left\{\mathbf{B}^{*}\right\}^{-1}\right]_{k j}=\left\langle J_{k}\left(\hat{\Psi}_{j}^{\mathrm{ext}}-\hat{\Psi}_{j}^{\mathrm{int}}\right)\right\rangle
$$

The calculation and inversion of the matrix are straightforward. The result is

$$
\mathbf{B}^{*}=\left[\begin{array}{cccc}
\frac{R}{\eta u_{0} d_{1}} & \frac{2 R\left(1+\frac{1}{4} R^{2} / \rho^{2}\right)}{\eta u_{0} d_{1}} & 0 & \frac{R^{2}}{\rho^{2} n u_{0} d_{1}} \\
0 & \frac{R}{6 \eta u_{0}} & 0 & 0 \\
0 & 0 & -\frac{R}{\lambda d_{2} T} & \frac{\frac{5}{2} R k_{B}}{\lambda d_{2}} \\
0 & 0 & 0 & -\frac{1}{n u_{0}}
\end{array}\right]
$$

with

$$
d_{1}=3+3 R / \rho+\frac{3}{2} R^{2} / \rho^{2}+\frac{1}{2} R^{3} / \rho^{3}, \quad d_{2}=2+2 R / \sigma+R^{2} / \sigma^{2} \quad .
$$

In calculating the matrix elements $\left[\left\{\mathbf{B}^{*}\right\}^{-1}\right]_{k j}$ defined by $(6.20)$ we neglected contributions due to velocity gradients occurring in $\hat{\Psi}_{3}$. This is consistent with the truncation of the normal solutions (4.16) at order $l / R$. The extension of $\mathbf{B}^{*}$ to $\mathbf{B}$ simply is done by adding the elements $B_{55}$ and $B_{66}$, which, as discussed above, are both unity. If we combine Eqs. (6.13) and (6.17), we see that Eq. (6.2) can now be represented in matrix form as

$$
(1-\mathbf{B A}) P \hat{\Psi}=\mathbf{B} S^{0}
$$

where 1 is the $6 \times 6$ unit matrix and the initial source $S^{0}$

$$
S^{0}=-P \tilde{\mathbf{T}} \beta m[\mathbf{v} \cdot \mathbf{V}(z)]
$$

is represented as a six-component vector with the expansion coefficients $\gamma_{i}$ from (6.16) as components.

Of the things that are still needed for an evaluation of (6.22), one is an explicit form of $S^{o}$, the other is an explicit form of the matrix $\mathbf{A}$. The former contains the operator $P \tilde{\mathbf{T}} P$ ( $\widetilde{\mathbf{T}}$ acts upon a hydrodynamic distribution), the latter consists of matrix elements of the operator $P \widetilde{\overline{\mathrm{T}}} P$. Neither operator can be calculated explicitly; both operators have to be represented by matrices containing a number of unknown coefficients. In order to make these representations consistent it is advantageous to rewrite $S^{0}$ in terms of $P \tilde{\mathbf{T}} P$. To do so we set $\mathbf{V}(z)=\hat{\mathbf{z}} \mathbf{V}(z)$ and start from the identity

$$
\begin{aligned}
\mathbf{T} \beta m[\mathbf{v} \cdot \mathbf{V}(z)] & =\overline{\mathbf{T}} \beta m[\mathbf{v} \cdot \mathbf{V}(z)]-\beta m z(\mathbf{v} \cdot \hat{\mathbf{r}})[\mathbf{r} \cdot \mathbf{V}(z)] \delta(r-R) \\
& +(\mathbf{v} \cdot \hat{\mathbf{r}}) \delta(r-R)\left[V(z) / u_{0}\right]\left\{\hat{\Psi}_{4}^{\text {ext }}-\hat{\Psi}_{4}^{\text {int }}\right\}
\end{aligned}
$$


which follows from (2.15), (6.5d), (5.3d), and (4.16). Next we use (4.22b) in order to construct $\widetilde{\mathbf{T}}$. The last term on the right-hand side of (6.24) does not contribute to the boundary layer corrections, because it is a hydrodynamic source of type (6.7). The first term on the right-hand side is transformed into $\tilde{\mathbf{T}} \beta m[\mathbf{v} \cdot \mathbf{V}(z)]$. The second term on the right-hand side gives rise to a source of radial momentum (a source of type $S_{1}$ ) and perhaps an energy source (of type $S_{3}$ ), but the strength of these sources is an order $l / R$ less than the strength of similar sources resulting from $\tilde{\overline{\mathbf{T}}} \beta m[\mathbf{v} \cdot \mathbf{V}(z)]$ (we use again that $z \sim v / R^{2}$ ). Henceforth the contributions resulting from this term should be neglected in our approximation. The resulting equation for $\widetilde{\mathbf{T}} \beta m[\mathbf{v} \cdot \mathbf{V}(z)]$ is

$$
\begin{aligned}
\tilde{\mathbf{T}} \beta m[\mathbf{v} \cdot \mathbf{V}(z)]= & \tilde{\mathbf{T}} \beta m[\mathbf{v} \cdot \mathbf{V}(z)] \\
& +(\mathbf{v} \cdot \hat{\mathbf{r}}) \delta(r-R)\left[V(z) / u_{0}\right]\left\{\hat{\Psi}_{4}^{\mathrm{ext}}-\hat{\Psi}_{4}^{\mathrm{int}}\right\}
\end{aligned}
$$

We make a separation

$$
S^{0}=S_{1}^{0}+S_{\mathrm{II}}^{0}
$$

with

$$
\begin{aligned}
S_{\mathrm{I}}^{0} & =-P \tilde{\mathbf{T}} \beta m[\mathbf{v} \cdot \mathbf{V}(z)] \\
S_{\mathrm{II}}^{0} & =-(\mathbf{v} \cdot \hat{\mathbf{r}}) \delta(r-R)\left[V(z) / u_{0}\right]\left\{\hat{\Psi}_{4}^{\mathrm{ext}}-\hat{\Psi}_{4}^{\mathrm{int}}\right\}
\end{aligned}
$$

With the aid of (6.12) and (6.14) these sources can be represented as vectors in the following way:

$$
S_{\mathrm{I}}^{0}=n V(z)\left(\begin{array}{c}
\lambda_{1} \\
\lambda_{3} \\
-\lambda_{4} \\
0 \\
S_{5} \\
S_{6}
\end{array}\right), \quad S_{\mathrm{II}}^{0}=V(z)\left(\begin{array}{c}
-\eta R / \rho^{2} \\
0 \\
\frac{5}{2} n / \beta \\
n \\
0 \\
0
\end{array}\right)
$$

where $S_{5}$ and $S_{6}$ are unknown constants.

Now we come back to the problem of the undetermined elements in the matrix $\mathbf{A}$. From the fact that $\hat{\Psi}$ vanishes inside the sphere it follows that $P \hat{\Psi}$ must be of the form

$$
P \hat{\Psi}=\sum_{i=1}^{4} \mu_{i} \hat{\Psi}_{i}
$$

because the inside parts of $\hat{\Psi}_{5}$ and $\hat{\Psi}_{6}$ cannot be canceled by linear combinations of the other $\hat{\Psi}_{i}$. This implies that in the vector equation 
(6.22) only the first four elements are physically relevant. These, however, are determined entirely by the $4 \times 4$ submatrices $\mathbf{B}^{*}$ and $\mathbf{A}^{*}$ with $A_{i j}^{*}=A_{i j}$ for $i, j \leqslant 4$ : First of all, since $P \hat{\Psi}$ is of the form (6.29) the elements $A_{i 5}$ and $A_{i 6}$ do not come into play in (6.22). Second, the elements $A_{5 i}$ and $A_{6 i}$ only contribute to the fifth and sixth elements of the vector equation, because of the property $B_{i j}=\delta_{i j}$ for $j=5,6$. These elements simply have to be such that, taking into account the unknown values of $S_{5}$ and $S_{6}{ }^{\prime}(6.22)$ becomes an identity for the fith and sixth element once it has been solved for the first four elements. The equation for the first four elements reduces to

$$
\left(1^{*}-\mathbf{B}^{*} \mathbf{A}^{*}\right) \hat{\Psi}^{*}=\mathbf{B}^{*}\left(S_{1}^{0 *}+S_{\mathrm{II}}^{0 *}\right)
$$

where $\hat{1}^{*}$ is the $4 \times 4$ unit matrix: $\hat{\Psi}^{*}$ is the restriction of $P \hat{\Psi}$ to a fourcomponent vector with the coefficients $\mu_{i}$ from (6.29) as components; and the $S^{0 *}$ are the four-component vectors

$$
S_{\mathrm{I}}^{0 *}=n V(z)\left(\begin{array}{c}
\lambda_{1} \\
\lambda_{3} \\
-\lambda_{4} \\
0
\end{array}\right), \quad S_{\mathrm{II}}^{0 *}=V(z)\left(\begin{array}{c}
-\eta R / \rho^{2} \\
0 \\
\frac{5}{2} n / \beta \\
n
\end{array}\right)
$$

The explicit form of $\mathbf{A}^{*}$ is obtained from (6.14) as

$$
\mathbf{A}^{*}=\left[\begin{array}{cccc}
-n \lambda_{1}\left(1+\frac{1}{3} R / \rho\right) u_{0} & 2 n \lambda_{1} u_{0} & n \lambda_{2}(1+R / \sigma) & 0 \\
-n \lambda_{3}\left(\frac{1}{2}-\frac{1}{6} R / \rho\right) u_{0} & -n \lambda_{3} u_{0} & -n \lambda_{3} D_{T} / \sigma & 0 \\
n \lambda_{4}\left(1+\frac{1}{3} R / \rho\right) u_{0} & -2 n \lambda_{4} u_{0} & n \lambda_{5}(1+R / \sigma) & 0 \\
0 & 0 & 0 & 0
\end{array}\right]
$$

where we used the fact that $\hat{\Psi}_{4}$ vanishes outside the sphere. Finally, we rewrite $(6.30)$ once more by putting

$$
\hat{\mathbf{\Psi}}^{*}=\mathbf{B}^{*} S^{*}=\mathbf{B}^{*} \sum_{i=1}^{4} C_{i} S_{i}
$$

defining $S^{*}$ as the hydrodynamic source of $\hat{\Psi}^{*}$. If we substitute this into (6.30) and multiply by $\left\{\mathbf{B}^{*}\right\}^{-1}$, we obtain

$$
\begin{aligned}
\left(1 *-\mathbf{A}^{*} \mathbf{B}^{*}\right) S^{*} & =S^{0 *} \\
S^{*} & =\left(1 *-\mathbf{A}^{*} \mathbf{B}^{*}\right)^{-1} S^{0 *} \\
& =\left(1 *-\mathbf{A}^{*} \mathbf{B}^{*}\right)^{-1} S_{\mathrm{I}}^{0 *}+S_{\mathrm{II}}^{0 *} \\
& =S_{\mathrm{I}}^{*}+S_{\mathrm{II}}^{*}
\end{aligned}
$$


To obtain $(6.34 \mathrm{c})$ we used the property

$$
\mathbf{A}^{*} \mathbf{B}^{*} S_{\mathrm{II}}^{0 *} \sim \mathbf{A}^{*}\left(\begin{array}{l}
0 \\
0 \\
0 \\
1
\end{array}\right)=0
$$

The solution of (6.34d) is obtained by elementary algebra from $(6.21),(6.31)$, and $(6.32)$ as

$$
S_{1}^{*}=V(z)\left(\begin{array}{c}
C_{1} \\
C_{2} \\
0 \\
0
\end{array}\right)
$$

with

$$
\begin{aligned}
C_{1}= & \frac{3}{2}\left[n \lambda_{3}\left(1+\frac{R}{\rho}+\frac{R^{2}}{\rho^{2}}\right)+\frac{\eta}{R}\left(6+\frac{6 R}{\rho}+\frac{3 R^{2}}{\rho^{2}}+\frac{R^{3}}{\rho^{3}}\right)\right] \\
& \times\left(3+\frac{R}{\rho}+n \lambda_{3} \frac{R}{\eta}\right)^{-1} \\
C_{2}= & \frac{3}{2} n \lambda_{3}\left(1+\frac{R}{\rho}\right)\left(3+\frac{R}{\rho}+n \lambda_{3} \frac{R}{\eta}\right)^{-1}
\end{aligned}
$$

In the diffuse reflection case $\lambda_{3}$ is of order unity. Then all terms not containing $\lambda_{3}$ must be consistently neglected since they are of relative order $l / R$. Equations (6.36) in that case reduce to

$$
\begin{aligned}
& C_{1}^{\mathrm{di}}=\frac{3}{2}(\eta / R)\left(1+R / \rho+R^{2} / \rho^{2}\right) \\
& C_{2}^{\mathrm{di}}=\frac{3}{2}(\eta / R)(1+R / \rho)
\end{aligned}
$$

In the case that $\overline{\mathbf{T}}=\overline{\mathbf{T}}_{\alpha}$ we can substitute (6.15) for $\lambda_{3}$. If in addition the viscous length (on the order of the mean free path)

$$
l_{v}=(2 \pi \beta m)^{1 / 2} v
$$

is introduced, Eqs. (6.36) take the form

$$
\begin{aligned}
C_{1}{ }^{\alpha}= & \frac{3}{2} \frac{\eta}{R}\left[\frac{\alpha R}{l_{v}}\left(1+\frac{R}{\rho}+\frac{R^{2}}{\rho^{2}}\right)+6+\frac{6 R}{\rho}+\frac{3 R^{2}}{\rho^{2}}+\frac{R^{3}}{\rho^{3}}\right] \\
& \times\left(3+\frac{R}{\rho}+\frac{\alpha R}{l_{v}}\right)^{-1}
\end{aligned}
$$




$$
C_{2}^{\alpha}=\frac{3}{2} \frac{\eta}{R} \frac{\alpha R}{l_{v}}\left(1+\frac{R}{\rho}\right)\left(3+\frac{R}{\rho}+\frac{\alpha R}{l_{v}}\right)^{-1}
$$

Notice that as soon as $\alpha \gg I_{v} / R,(6.39)$ reduces to $(6.37)$.

The distribution function $\hat{\Psi}$ is found from (6.33) in the two respective cases as

$$
\begin{aligned}
P \hat{\Psi}_{d i}= & \frac{3}{2} \frac{V(z)}{u_{0}}\left[\hat{\Psi}_{1}+\frac{1}{6}\left(1+\frac{R}{\rho}\right) \hat{\Psi}_{2}-\frac{2}{3} \hat{\Psi}_{4}\right] \\
P \hat{\Psi}_{\alpha}= & \frac{3}{2} \frac{V(z)}{u_{0}\left(3+R / \rho+\alpha R / I_{v}\right)} \\
& \times\left[\left(\frac{\alpha R}{l_{v}}+2\right) \hat{\Psi}_{1}+\frac{\alpha R}{6 l_{v}}\left(1+\frac{R}{\rho}\right) \hat{\Psi}_{2}\right]-\frac{V(z)}{u_{0}} \hat{\Psi}_{4}
\end{aligned}
$$

Notice that in either case $P \hat{\Psi}$ vanishes inside the sphere, as it should.

The drag force exerted by the gas molecules on the sphere can be obtained from (3.14) as

$$
\mathbf{F}(z)=-\int d \mathbf{r}\langle m \mathbf{v} \overline{\mathbf{T}}\{-\beta m[\mathbf{v} \cdot \mathbf{V}(z)]+\hat{\Psi}(\mathbf{r}, \mathbf{v}, z)\}\rangle
$$

With the aid of (2.15), (4.17), (4.20), (4.22), and (6.2), we find from Eq. (6.41)

$$
\begin{aligned}
\mathbf{F}(z)= & -\int d \mathbf{r}\langle m \mathbf{v}\{P \tilde{\mathbf{T}} P \hat{\Psi}-P \tilde{\mathbf{T}} P \beta m[\mathbf{v} \cdot \mathbf{V}(z)] \\
& -(\mathbf{v} \cdot \hat{\mathbf{r}}) \delta(r-R) \beta m[\mathbf{v} \cdot \mathbf{V}(z)]\}\rangle
\end{aligned}
$$

The third term on the right-hand side of (6.42) gives a vanishing contribution to the integral, and from (6.1) it follows that $P \tilde{\tilde{\mathbf{T}}} P \hat{\Psi}-P \tilde{\mathbf{T}} \beta m[\mathbf{v} \cdot \mathbf{V}(z)]$ can be identified with the source $S^{*}$ of $P \hat{\Psi}$ which appears in Eq. (6.33). Hence the expression for the drag force can be rewritten,

$$
\begin{aligned}
\mathbf{F}(z)= & -\int d \mathbf{r}\left\langle m[(\mathbf{v} \cdot \hat{\mathbf{r}})(\hat{\mathbf{r}} \cdot \hat{\mathbf{z}})+(\mathbf{v} \cdot \hat{\boldsymbol{\Theta}})(\hat{\boldsymbol{\Theta}} \cdot \hat{\mathbf{z}})] \hat{\mathbf{z}} S^{*}\right\rangle \\
= & -\int d \mathbf{r} \hat{\mathbf{z}}\left[\left(C_{1}-\frac{\eta R}{\rho^{2}}\right) \cos ^{2} \Theta+C_{2} \sin ^{2} \Theta\right] V(z) \delta(r-R) \\
= & -\frac{4 \pi R^{2}}{3} \hat{\mathbf{z}}\left(C_{1}-\frac{\eta R}{\rho^{2}}+2 C_{2}\right) V(z) \\
= & -2 \pi \eta R \mathbf{V}(z)\left[\frac{\alpha R}{l_{v}}\left(3+\frac{3 R}{\rho}+\frac{1}{3} \frac{R^{2}}{\rho^{2}}\right)+6+\frac{6 R}{\rho}+\frac{R^{2}}{\rho^{2}}+\frac{1}{3} \frac{R^{3}}{\rho^{3}}\right] \\
& \times\left(3+\frac{R}{\rho}+\frac{\alpha R}{l_{v}}\right)^{-1}
\end{aligned}
$$


To obtain (6.43a) we used rotational symmetry about the $z$ axis; to derive $(6.43 b)$ one needs $(6.4),(6.8),(6.34 c),(6.34 d),(6.35)$, and (6.31) and then one arrives at $(6.43 \mathrm{~d})$ by means of elementary algebra. The result $(6.43 \mathrm{~d})$ agrees with the expression for the drag force obtained by Albano et al. ${ }^{(21)}$ from the linearized Navier-Stokes equations, provided one identifies $\alpha R / l_{v}$ with the slip coefficient. Hence we may conclude that the particle-sphere reflection mechanism described by $\overline{\mathbf{T}}_{\alpha}$ gives rise to partial slip boundary conditions with slip coefficient $\alpha R / l_{v}$. We want to stress again that this is only true as long as $\alpha$ is of order $l / R$, and in all realistic cases stick boundary conditions will emerge, up to corrections of order $l / R$. It is worth emphasizing the fact that Eq. (6.43d) leads to $\mathbf{F}=-6 \pi \eta R \mathbf{V}$ for diffuse reflection, and to $F=-4 \pi \eta R V$ for specular reflection, in the case that the flow is steady and $z$ can be set equal to zero. These results should be contrasted with those described at the end of Section 3 that are obtained if the boundary layer is not taken into account. Finally we mention that the drag force can also be calculated in a slightly different way by using the equality

$$
\overline{\mathbf{T}}\{-\beta m[\mathbf{v} \cdot \mathbf{V}(z)]+\hat{\Psi}\}=(\mathbf{v} \cdot \hat{\mathbf{r}}) \delta(r-R)\{-\beta m[\mathbf{v} \cdot \mathbf{V}(z)]+\hat{\Psi}\}
$$

which follows from (2.15), (2.18), and (4.4). Substituting this into (6.41), one obtains

$$
\begin{aligned}
\mathbf{F}(z) & =-\int d \mathbf{r}\langle m \mathbf{v}(\mathbf{v} \cdot \hat{\mathbf{r}}) \delta(r-R)\{-\beta m[\mathbf{v} \cdot \mathbf{V}(z)]+\hat{\Psi}\}\rangle \\
& =-\int d \mathbf{r}\langle m \mathbf{v}(\mathbf{v} \cdot \hat{\mathbf{r}}) \delta(r-R) P \hat{\Psi}\rangle
\end{aligned}
$$

where we used that the average value of the radial current $m \mathbf{v}(\mathbf{v} \cdot \hat{\mathbf{r}})$ is determined to leading order in $l / R$ by the hydrodynamic part of the distribution function alone, as was discussed in Section 5. Evaluation of $(6.44 \mathrm{~b})$ with the aid of (6.40) leads to the same result as $(6.43 \mathrm{~d})$.

We conclude this section with the following remark: It might appear that we have really accomplished very little after all this work, simply giving a rather long derivation of a well-known result--Stokes' law-and of the Chapman-Enskog normal solution for the hydrodynamic part of the distribution function $P \hat{\Psi}$. However, we showed at the end of Section 3 that it is not a simple matter to obtain Stokes' law from Eq. (6.41). The effects of the boundary layer must be taken into account somehow when the force is computed, and what is what we accomplished here.

We have taken the boundary layer effects into account in this section by either of two ways. One way is to use the relation between the boundary layer distribution function and the hydrodynamic distribution 
function to upgrade the $\overline{\mathbf{T}}$ in Eq. (6.41) to the $\tilde{\widetilde{\mathbf{T}}}$ and $\tilde{\mathbf{T}}$ in Eq. (6.42). The other way is to use the fact that $P \hat{\Psi}$ determines the radial currents to leading nonvanishing order in $l / R$, from which we derive (6.44b). In any case, a careful treatment of the boundary layer is required.

\section{THE CONTINUUM LIMIT}

In spite of all the criticism raised in Section 5 against the projection operator defined by (4.10) or (4.13), one may ask if, under certain limiting circumstances, this projection operator might still be useful for obtaining the hydrodynamic part of an arbitrary function of velocity and position. Suppose, for example, that all lengths are scaled by dividing them by the characteristic macroscopic length $R$, and that all times are scaled by dividing them by the characteristic time $R^{2} / v$, and then one takes the limit $R \rightarrow \infty$, keeping the unscaled mean free path length and mean free time fixed. In this limit, which is called the continuum limit, the thickness of the kinetic boundary layer shrinks to zero, so effectively the kinetic boundary layer becomes part of the surface of the sphere. In this limit the distribution function outside the sphere consists of a normal part alone. Furthermore, the particle-sphere collision operator $\overline{\mathbf{T}}$ has to be replaced by an operator $\overline{\mathbf{T}}_{\text {eff }}$, which also has the general form (2.3), but with a kernel $P_{\text {eff }}\left(\mathbf{v}^{\prime}, \mathbf{v}, \hat{\boldsymbol{\sigma}}\right)$ describing the probability that a gas molecule entering the kinetic boundary layer with velocity $\mathbf{v}^{\prime}$ at position $R \hat{\sigma}$ leaves the kinetic boundary layer with velocity $\mathbf{v}$. Microscopically seen, the molecule will not leave the kinetic boundary layer at the same position and time as it entered. The point of exit will be at a distance on the order of the mean free path removed from the point of entrance and the moment of exit will be on the order of the mean free time later than the moment of entrance. In the continuum limit, however, the displacement is scaled by $R$ and it is reduced to zero. Furthermore, the typical macroscopic time scale is $R^{2} / v$, as discussed before; hence the time delay is also scaled down to zero in the continuum limit.

To specify more precisely what is meant by entering and leaving the kinetic boundary layer we consider a shell of thickness $\epsilon R$ around the sphere, which entirely contains the kinetic boundary layer, provided $R$ is large enough. Then the distribution function outside the shell is always a hydrodynamic distribution function of the form (4.16). We now define $P_{\text {eff }}\left(\mathbf{v}^{\prime}, \mathbf{v}, \hat{\boldsymbol{\sigma}}\right)$ by the relation

$$
P_{\text {eff }}\left(\mathbf{v}^{\prime}, \mathbf{v}, \hat{\boldsymbol{\sigma}}\right)=\lim _{\epsilon \rightarrow 0} \lim _{R \rightarrow \infty} P_{\epsilon}\left(\mathbf{v}^{\prime}, \mathbf{v}, \hat{\boldsymbol{\sigma}}\right)
$$

where $P_{\epsilon}$ describes the probability that a gas molecule entering the shell $R<r<(1+\epsilon) R$ at the position $(1+\epsilon) R \hat{\sigma}$ with velocity $\mathbf{v}^{\prime}$ leaves this shell 
with velocity $\mathbf{v}$. Since the outside distribution function is entirely hydrodynamic, $\overline{\mathbf{T}}_{\text {eff }}$ acts on normal distributions only and, if one considers $\overline{\mathbf{T}}_{\text {eff }} f$ as a source term, the source term generates a hydrodynamic distribution only. In other words: $\overline{\mathbf{T}}_{\mathrm{eff}}=P \overline{\mathbf{T}}_{\mathrm{eff}} P$. In fact the whole relevant space of distribution functions in the continuum limit consists of the hydrodynamic distributions alone; hence the use of the projection operator is in a way superfluous. All operators acting on the distribution function simply have to transform hydrodynamic distributions into hydrodynamic distributions.

On comparison with (4.21) we may conclude that we can make the identification

$$
\overline{\mathbf{T}}_{\mathrm{eff}}=\lim _{R \rightarrow \infty} P \tilde{\mathbf{T}} P
$$

where in fact one has to use the properly scaled form of $P \tilde{\mathbf{T}} P$. The latter operator describes the source of a normal solution resulting from $\overline{\mathbf{T}}$ acting on a given normal solution plus the boundary layer induced by it. This is precisely what we require of $\overline{\mathbf{T}}_{\text {eff }}$. However, in the continuum limit all detailed information of the boundary layer distribution functions is lost and all that remains is the information about the particular hydrodynamic solution generated by the action of $\overline{\mathbf{T}}_{\text {eff }}$ on a given hydrodynamic solution. This action can now be characterized by means of the projection operator (4.13): because of the scaling of all lengths by $R$, the upper limit on the $\mathbf{k}$ integration in (4.10) goes to infinity and the smeared-out $\Delta$ functions, such as the one occurring in (4.12) are reduced to ordinary $\delta$ functions. Hence one has

$$
\left.\overline{\mathbf{T}}_{\mathrm{eff}} \hat{\Psi}_{\mathrm{NS}}=P \overline{\mathbf{T}}_{\mathrm{eff}} \hat{\Psi}_{\mathrm{NS}}=\sum_{\nu} \mid \Psi^{\gamma}(\mathbf{v})\right)\left(\Psi^{\gamma}(\mathbf{v}) \mid \overline{\mathbf{T}}_{\mathrm{eff}} \hat{\Psi}_{\mathrm{NS}}\right)
$$

As in (6.14), the matrix elements of $\overline{\mathbf{T}}_{\text {eff }}$ can be determined up to some unknown multiplicative constants by the methods of Appendix B. It will turn out, however, for reasons to be discussed later, that it does not suffice to approximate the $\Psi^{\gamma}$, which are given by an expansion in terms of the gradients, by the lowest order terms alone; the terms proportional to the gradient also have to be included. The latter give rise to a new type of source proportional to a gradient ${ }^{11}$ of the function, $\delta^{\prime}(r-R)$. On the other hand, inclusion of terms of $O(\nabla)$ in $\hat{\Psi}_{\mathrm{NS}}$ is not needed in (7.3); these would lead only to corrections of $O(l / R)$ (zero in the continuum limit) in coefficients

${ }^{11}$ In spite of the fact that all sources in the extended Boltzmann equation should be proportional to $\delta(r-R)$, it is not inconsistent to have such gradient sources here. The reason is that the projection operator $P$, Eq. (4.10), smears out the $\delta$ function over a few mean free paths; then in the continuum limit such a smeared-out $\delta$ function can be expanded as a multipole expansion in terms of $\delta(r-R)$ and its derivatives. 
of source terms which were nonzero already. For brevity we restrict ourselves to the matrix elements of $\overline{\mathbf{T}}_{\alpha, \text { eff }}$ with $\alpha \sim l / R$ as before, which follow from Eqs. (B.3), (4.15), (4.16), (2.10), and (2.11) as

$$
\begin{aligned}
\left\langle\Psi^{n} \overline{\mathbf{T}}_{\alpha, \mathrm{eff}} \hat{\boldsymbol{\Psi}}_{\mathrm{NS}}\right\rangle= & 0 \\
\left\langle\Psi^{T} \overline{\mathbf{T}}_{\alpha, \mathrm{eff}} \hat{\boldsymbol{\Psi}}_{\mathrm{NS}}\right\rangle= & n\left(\frac{2}{3}\right)^{1 / 2}\left\{\delta(r-R)\left[\beta \lambda_{4}(\mathbf{u} \cdot \hat{\mathbf{r}})-\frac{2 \alpha}{(2 \pi \beta m)^{1 / 2}} \frac{\delta T}{T}\right]\right. \\
& \left.-\frac{5}{2} D_{T}(\boldsymbol{\nabla} \cdot \hat{\mathbf{r}}) \delta(r-R) \frac{\delta T}{T}\right\} \\
& +\lambda_{6}(\mathbf{V} \cdot \hat{\mathbf{r}}) \delta(r-R)(\mathbf{u} \cdot \hat{\mathbf{r}})+O\left(\frac{l}{R}\right)^{2} \\
\left\langle\Psi^{n} \overline{\mathbf{T}}_{\alpha, \mathrm{eff}} \hat{\Psi}_{\mathrm{NS}}\right\rangle= & -n\left(\frac{\beta}{m}\right)^{1 / 2}\left\{\delta(r-R)\left[\lambda_{1}(\mathbf{u} \cdot \hat{\mathbf{r}})+\lambda_{2}\left(\frac{\delta T}{T}\right)\right] \hat{\mathbf{r}}\right\} \\
& +\alpha(2 \pi)^{-1 / 2} \delta(r-R)(\mathbf{u} \cdot \hat{\mathbf{\Theta}}) \hat{\boldsymbol{\Theta}} \\
& +v(\beta m)^{1 / 2} \boldsymbol{\nabla} \cdot(\hat{\mathbf{r}} \hat{\mathbf{\Theta}}+\hat{\mathbf{\Theta}} \hat{\mathbf{r}}) \delta(r-R)(\mathbf{u} \cdot \hat{\boldsymbol{\Theta}})+O\left(\frac{l}{R}\right)^{2}
\end{aligned}
$$

Here $\lambda_{1}, \lambda_{2}$, and $\lambda_{4}$ are the same unknown constants as in (6.14), and $\lambda_{6}$ is a new unknown constant coupling the energy density to a gradient source. Now we want to know the distribution function $\hat{\Psi}_{\mathrm{NS}}$ generated by the source $\overline{\mathbf{T}}_{\alpha, \text { eff }} \hat{\Psi}_{\mathrm{NS}}^{0}$ for a given $\hat{\Psi}_{\mathrm{NS}}^{0}$.

To do this we first discuss the normal solutions of the linearized Boltzmann equation with a general inhomogeneous or source term $S$; next we pass to the case where $S$ is identified with $\widetilde{\mathbf{T}}_{\alpha, \text { eff }} \hat{\Psi}_{\mathrm{NS}}^{0}$. This Boltzmann equation is of the form

$$
(z+\mathbf{v} \cdot \nabla-L) \hat{\Psi}_{\mathrm{NS}}=S
$$

Because we work in the continuum limit, only the action on the normal solution is needed. If one now inserts (4.16) for $\hat{\Psi}_{N S}$ and takes inner products with $\Psi^{n}, \Psi^{u}$, and $\Psi^{T}$, one obtains, with the aid of (4.15), the hydrodynamic equations in the presence of source terms

$$
\begin{array}{r}
z \delta n+n \nabla \cdot \mathbf{u}=\sigma_{n} \\
z n m \mathbf{u}+\beta^{-1}[\nabla \delta n+(n / T) \nabla \delta T]-\eta\left[\nabla^{2} \mathbf{u}+\frac{1}{3} \nabla(\nabla \cdot \mathbf{u})\right]=\sigma_{p} \\
z \frac{\delta T}{T}+\frac{2}{3} \nabla \cdot \mathbf{u}-\frac{5}{3} \frac{D_{T}}{T} \nabla^{2} \delta T=\frac{2 \beta}{3 n} \sigma_{e}-\frac{1}{n} \sigma_{n} \equiv \frac{2 \beta}{3 n}\left(\sigma_{h}+\frac{1}{\beta} \sigma_{n}\right)
\end{array}
$$

where the density source, the momentum source, and the energy source are 
defined by the relations

$$
\begin{aligned}
\sigma_{n} & =n\left(\Psi^{n} \mid S\right) \\
\sigma_{p} & =n(m / \beta)^{1 / 2}\left(\Psi^{u} \mid S\right) \\
\sigma_{e} & =\left(\frac{3}{2}\right)^{1 / 2}(n / \beta)\left(\Psi^{T} \mid S\right)+\frac{3}{2}(n / \beta)\left(\Psi^{n} \mid S\right)
\end{aligned}
$$

and $\sigma_{h}$ is defined to be the enthalpy source.

Once a solution of (7.6) has been found the corresponding solution of (7.5) follows with (4.16); as a consequence of the restriction to normal solutions, Eqs. (7.5) and (7.6) are entirely equivalent. In order to solve (7.6) we must know what forms the source terms occurring in these equations can possibly take. From the tensorial arguments developed in Section 5 we may conclude that the fields $\mathbf{u}, \delta T$, and $\delta n$ occurring in the solution of the extended Boltzmann equation must be of the form

$$
\begin{gathered}
\mathbf{u}=f_{1}(r) \cos \Theta \hat{\mathbf{r}}+f_{2}(r) \sin \Theta \hat{\Theta} \\
\delta T=f_{\mathfrak{3}}(r) \cos \Theta, \quad \delta n=f_{4}(r) \cos \Theta
\end{gathered}
$$

If we make the identification $S=\overline{\mathbf{T}}_{\alpha, \mathrm{eff}} \Psi_{\mathrm{NS}}$, the following possibilities for the hydrodynamic source terms are obtained from (7.4) and (7.7). There are three momentum sources:

$$
\begin{aligned}
\boldsymbol{\sigma}_{1} & =(\hat{\mathbf{z}} \cdot \hat{\mathbf{r}}) \hat{\mathbf{r}} \delta(r-R) \\
\boldsymbol{\sigma}_{2} & =\hat{\mathbf{z}} \cdot(\mathbb{1}-\hat{\mathbf{r}} \hat{\mathbf{r}}) \delta(r-R)=(\hat{\mathbf{z}} \cdot \hat{\boldsymbol{\Theta}}) \hat{\Theta} \delta(r-R) \\
\boldsymbol{\sigma}_{4} & =\nabla \cdot\{[\hat{\mathbf{r}} \hat{\mathbf{z}}+\hat{\mathbf{z}} \hat{\mathbf{r}}-\mathbf{2} \hat{\mathbf{r}} \hat{\mathbf{r}}(\hat{\mathbf{z}} \cdot \hat{\mathbf{r}})] \delta(r-R)\} \\
& =\nabla \cdot\{(\hat{\boldsymbol{\Theta}} \hat{\mathbf{r}}+\hat{\mathbf{r}} \hat{\boldsymbol{\Theta}}) \sin \Theta \delta(r-R)\}
\end{aligned}
$$

and two enthalpy sources:

$$
\begin{aligned}
& \sigma_{3}=(\hat{\mathbf{z}} \cdot \hat{\mathbf{r}}) \delta(r-R) \\
& \sigma_{5}=\nabla \hat{\mathbf{z}}: \hat{\mathbf{r}} \hat{\mathbf{r}} \delta(r-R)
\end{aligned}
$$

Finally, if we also consider the sources that can result from the action of $\mathbf{T}_{\alpha, \mathrm{eff}}$ on a normal solution, we find an additional momentum source

$$
\sigma_{4}{ }^{\prime}=\nabla \cdot\left[\left(\hat{\mathbf{r}} \hat{\mathbf{r}}-\frac{1}{3} \mathbb{1}\right)(\hat{\mathbf{r}} \cdot \hat{\mathbf{z}}) \delta(r-R)\right]
$$

and a density source

$$
\sigma_{6}=(\hat{\mathbf{z}} \cdot \hat{\mathbf{r}}) \delta(r-R)
$$

The source $\sigma_{4}{ }^{\prime}$ accidentally does not occur in (7.4), because $(\mathbf{v} \cdot \hat{\mathbf{r}})^{2}$ is a left eigenfunction of $\overline{\mathbf{T}}_{\mathrm{sp}}$. Furthermore, $\sigma_{4}{ }^{\prime}$ is effectively equal to a linear 
combination of $\sigma_{1}$ and $\sigma_{2}$ :

$$
\sigma_{4}^{\prime}=\left(2 \sigma_{1}-\sigma_{2}\right) / R
$$

as is shown in Appendix D.

The hydrodynamic fields resulting from any of the sources $\sigma_{1}, \ldots, \sigma_{6}$ can be calculated from (7.6). Again, the corresponding normal distribution functions are always linear combinations of the distributions $\hat{\Psi}_{1}, \ldots, \hat{\Psi}_{6}$ given in Section 6. For simplicity we will restrict ourselves from now on to the stationary case, $z=0$, but this restriction is in no way essential and can be avoided without problems. In the stationary case the form of $\hat{\Psi}_{1}, \ldots, \hat{\Psi}_{6}$ simplifies and these basic functions are described by the following set of fluid fields:

$$
\begin{aligned}
\hat{\Psi}_{1}: \quad \mathbf{u}= & u_{0}\{W(r)(R / 2 r)[\hat{\mathbf{z}}+(\hat{\mathbf{z}} \cdot \hat{\mathbf{r}}) \hat{\mathbf{r}}]+[1-W(r)] \hat{\mathbf{z}}\} \\
\delta n / n= & W(r) \beta m v u_{0}\left(R / r^{2}\right)(\hat{\mathbf{z}} \cdot \hat{\mathbf{r}}) \\
\delta T / T= & 0 \\
\hat{\Psi}_{2}: \quad \mathbf{u}= & u_{0}\left\{W(r)\left(R^{3} / r^{3}\right)[\hat{\mathbf{z}}-3(\hat{\mathbf{z}} \cdot \hat{\mathbf{r}}) \hat{\mathbf{r}}]-2[1-W(r)] \hat{\mathbf{z}}\right\} \\
\delta n / n= & \delta T / T=0 \\
\hat{\Psi}_{3}: \quad \mathbf{u}= & \mathbf{0} \\
\delta n / n= & -\delta T / T=W(r)\left(R^{2} / r^{2}\right)(\hat{\mathbf{z}} \cdot \hat{\mathbf{r}}) \\
\hat{\Psi}_{4}: \quad \mathbf{u}= & u_{0}[1-W(r)] \hat{\mathbf{z}} \\
\delta n / n= & \delta T / T=0 \\
\hat{\Psi}_{5}: \quad \mathbf{u}= & u_{0}\left\{W(r) \frac{1}{30}\left(R^{3} / r^{3}\right)[\hat{\mathbf{z}}-3(\hat{\mathbf{z}} \cdot \hat{\mathbf{r}}) \hat{\mathbf{r}}]\right. \\
& \left.+[1-W(r)]\left(r^{2} / R^{2}\right)\left[-\frac{2}{15} \hat{\mathbf{z}}+\frac{1}{15}(\hat{\mathbf{z}} \cdot \hat{\mathbf{r}}) \hat{\mathbf{r}}\right]\right\} \\
\delta n / n= & -[1-W(r)] \frac{2}{3} \beta m v u_{0}\left(r / R^{2}\right)(\hat{\mathbf{z}} \cdot \hat{\mathbf{r}}) \\
\delta T / T= & 0 \\
\hat{\Psi}_{6}: \quad \mathbf{u}= & 0 \\
\delta n / n= & -\delta T / T=W(r) \frac{1}{4}\left(R^{2} / r^{2}\right)(\hat{\mathbf{z}} \cdot \hat{\mathbf{r}})-[1-W(r)] \frac{1}{2}(r / R)(\hat{\mathbf{z}} \cdot \hat{\mathbf{r}})
\end{aligned}
$$

The calculation from (7.6) of the stationary hydrodynamic fields generated by any of the sources $\sigma_{1}, \ldots, \sigma_{6}$ is tedious but straightforward. An outline and some of the details are given in Appendix D.

In the linearized hydrodynamic equations (7.6a)-(7.6c) certain combinations of the $\sigma_{i}$ appear which we have called $\sigma_{n}, \sigma_{p}$, amd $\sigma_{h}$. These are related to $\sigma_{1}, \ldots, \sigma_{6}$ by linear relations of the form

$$
\sigma_{n}=C_{6} \sigma_{6}
$$




$$
\begin{aligned}
& \sigma_{p}=C_{1} \sigma_{1}+C_{2} \sigma_{2}+C_{4} \sigma_{4} \\
& \sigma_{h}=C_{3} \sigma_{3}+C_{5} \sigma_{5}
\end{aligned}
$$

The coefficients $C_{1}, \ldots, C_{6}$ determine the source $S$ of the hydrodynamic distribution function, which follows from Eqs. (7.7), (4.13), and (7.8) as

$$
\begin{aligned}
S= & \frac{1}{n} \Psi^{n} C_{6} \sigma_{6}+\frac{1}{n}\left(\frac{\beta}{m}\right)^{1 / 2} \Psi^{u} \cdot\left(C_{1} \sigma_{1}+C_{2} \sigma_{2}+C_{4} \sigma_{4}\right) \\
& +\left(\frac{2}{3}\right)^{1 / 2} \frac{\beta}{n} \Psi^{T}\left(C_{3} \sigma_{3}+C_{5} \sigma_{5}+C_{6} \sigma_{6}\right)
\end{aligned}
$$

The hydrodynamic distribution function generated by such a source can be calculated in the following way: First calculate the hydrodynamic flow fields generated by each of the sources $\sigma_{1}, \ldots, \sigma_{6}$ by solving the equations (7.6) with all but one of the $C_{1}$ set equal to zero in Eq. (7.11). The flow fields generated by the set $\sigma_{n}, \sigma_{p}, \sigma_{e}$ are then found as a linear superposition of these. Finally the hydrodynamic distribution function generated by $S$ is found by substituting this solution into (4.16).

As a basis $S_{j}^{c}$ for the set of sources for hydrodynamic distribution functions ${ }^{12}$ we choose a set of six sources $S_{j}^{c}$ given by (7.12) with $C_{i}=\delta_{i j}$. The superscript $c$ refers to the continuous limit. A matrix $\mathbf{B}^{c}$ can then be defined again, such that $B_{i j}^{c}$ is the amount of $\hat{\Psi}_{i}$ generated by the source $S_{j}^{c}$ This matrix reads

$$
\mathbf{B}^{c}=\left[\begin{array}{cccccc}
R / 3 \eta u_{0} & 2 R / 3 \eta u_{0} & 0 & 0 & 0 & 0 \\
0 & R / 6 \eta u_{0} & 0 & 0 & 0 & -1 / 3 n u_{0} \\
0 & 0 & -R / 2 \lambda T & 0 & 0 & 0 \\
0 & 0 & 0 & 0 & 0 & 1 / n u_{0} \\
R / \eta u_{0} & -R / \eta u_{0} & 0 & -6 / \eta u_{0} & 0 & 0 \\
0 & 0 & 2 R / 3 \lambda T & 0 & 4 / 3 \lambda T & 0
\end{array}\right]
$$

From (7.7), (7.4), and (7.10) defining the $\hat{\Psi}_{i}$, and the definition of $S_{j}^{c}$ given below Eq. (7.12), we can infer the action of $\overline{\mathbf{T}}_{\alpha, \text { eff }}$ on any of the $\hat{\Psi}_{i}$. As in Section 6, this can be summarized in the form of a matrix $\mathbf{A}^{c}$ such that $A_{i j}^{c}$ is the amount of source $S_{i}^{c}$ generated by $\overline{\mathbf{T}}_{\alpha, \text { eff }}$ acting on $\hat{\Psi}_{j} \cdot$ The

\footnotetext{
${ }^{12}$ These should not be confused with the sources $\sigma_{1}, \ldots, \sigma_{6}$ of hydrodynamic fields $\delta n, \mathbf{u}, \delta T$.
} 
elements of this matrix are obtained as

$$
\mathbf{A}^{c}=\left[\begin{array}{cccccc}
-\lambda_{1}\left(\frac{m}{\beta}\right)^{1 / 2} n u_{0} & 2 \lambda_{1}\left(\frac{m}{\beta}\right)^{1 / 2} n u_{0} & \frac{\lambda_{2}(m / \beta)^{1 / 2} n}{k_{\mathrm{B}} \beta} & 0 & \lambda_{1}\left(\frac{m}{\beta}\right)^{1 / 2} n u_{0} & \frac{\lambda_{2}(m / \beta)^{1 / 2} n}{4 k_{\mathrm{B}} \beta} \\
\frac{-\alpha m n u_{0}}{2(2 \pi \beta m)^{1 / 2}}, & \frac{-\alpha m n u_{0}}{(2 \pi \beta m)^{1 / 2}} & 0 & 0 & \frac{-\alpha m n u_{0}}{30(2 \pi \beta m)^{1 / 2}} & 0 \\
\lambda_{4} n u_{0} & -2 \lambda_{4} n u_{0} & \frac{2 \alpha n}{\beta(2 \pi \beta m)^{1 / 2}} & 0 & \frac{-\lambda_{4} n u_{0}}{15} & \frac{\alpha n}{2 \beta(2 \pi \beta m)^{1 / 2}} \\
-\frac{1}{2} \eta u_{0} & -\eta u_{0} & 0 & 0 & \frac{-\eta u_{0}}{30} & 0 \\
\frac{\lambda_{6} n u_{0}}{\beta} & \frac{-2 \lambda_{6} n u_{0}}{\beta} & \lambda T & 0 & \frac{-\lambda_{6} n u_{0}}{15 \beta} & \frac{\lambda T}{4} \\
0 & 0 & 0 & 0 & 0 & 0
\end{array}\right]
$$

The extended Boltzmann equation (6.1) now assumes the form

$$
(\mathbf{v} \cdot \nabla-L) \hat{\Psi}_{\mathrm{NS}}=\overline{\mathbf{T}}_{\alpha, \mathrm{eff}} \hat{\Psi}_{\mathrm{NS}}-\mathbf{T}_{\alpha, \mathrm{eff}} \beta \mathrm{m}(\mathbf{v} \cdot \mathbf{V})
$$

which can be brought in a form like that of Eq. (6.22).

$$
\left(\mathbf{B}^{c}\right)^{-1} \hat{\Psi}_{\mathrm{NS}}=\mathbf{A}^{c} \hat{\Psi}_{\mathrm{NS}}+S^{0, c}
$$

The explicit form of $S^{0, c}$ is readily obtained by separating it according to

$$
S^{0, c}=-\overline{\mathbf{T}}_{\alpha, \mathrm{eff}} \beta m(\mathbf{v} \cdot \mathbf{V})+\beta m(\mathbf{v} \cdot \hat{\mathbf{r}})(\mathbf{v} \cdot \mathbf{V}) \delta(r-R)=S_{1}^{0, c}+S_{11}^{o, c}
$$

where (2.15) has been used. $S_{\mathrm{I}}^{0, c}$ is evaluated with the aid of $(7.4),(7.7)$, and (7.8) and $S_{\mathrm{II}}^{0, c}$ is calculated explicitly by taking inner products with $\left.\mid \Psi^{u}\right)$ and $\left.\mid \Psi^{T}\right)$. Representing a source $\sum_{i=1}^{6} C_{i} S_{i}$ by a column vector with elements $C_{i}$, and choosing the $z$ direction parallel to $V$ as before, one then obtains

$$
S_{\mathrm{I}}^{0, c}=\eta V\left(\begin{array}{c}
\gamma_{1} \\
\gamma_{6} \\
-\frac{1}{4} \gamma_{3} \\
1 \\
-\frac{2}{3} \gamma_{5} \\
0
\end{array}\right)
$$




$$
S_{\mathrm{II}}^{0, c}=\eta V\left(\begin{array}{c}
4 / R \\
-2 / R \\
0 \\
1 \\
0 \\
-n / \eta
\end{array}\right)
$$

where we introduced the constants

$$
\begin{array}{ll}
\gamma_{1}=\left(\lambda_{1} n / \eta\right)(m / \beta)^{1 / 2}, & \gamma_{2}=-\left(\lambda_{2} n / \lambda\right)(m / \beta)^{1 / 2} \\
\gamma_{3}=4 \lambda_{4} n / \eta, & \gamma_{4}=\alpha n k_{\mathrm{B}} /\left[\lambda(2 \pi \beta m)^{1 / 2}\right] \\
\gamma_{5}=\frac{2}{3} \lambda_{6} n / \beta \eta, & \gamma_{6}=\alpha m n /\left[\eta(2 \pi \beta m)^{1 / 2}\right]
\end{array}
$$

Furthermore, we used the identity (7.9). Like (6.22), Eq. (7.16) can be solved as

$$
\hat{\Psi}_{\mathrm{NS}}=\mathbf{B}^{c} S^{c}
$$

with

$$
S^{c}=\left(1-\mathbf{A}^{c} \mathbf{B}^{c}\right)^{-1} S^{0, c}=\left(1-\mathbf{A}^{c} \mathbf{B}^{c}\right)^{-1}\left(S_{\mathrm{I}}^{0, c}+S_{\mathrm{II}}^{0, c}\right) \equiv S_{\mathrm{I}}^{c}+S_{\mathrm{II}}^{c}
$$

The matrix $\mathbf{A}^{c} \mathbf{B}^{c}$ follows from (7.13)-(7.14) as

$\mathbf{A}^{c} \mathbf{B}^{c}=\left[\begin{array}{cccccc}-\frac{4}{15} R \gamma_{1} & -\frac{2}{5} R \gamma_{1} & \frac{1}{3} R \gamma_{2} & -\frac{2}{5} \gamma_{1} & -\frac{1}{3} \gamma_{2} & -\frac{2}{3} \eta \gamma_{1} / n \\ -\frac{1}{5} R \gamma_{6} & -\frac{7}{15} R \gamma_{6} & 0 & \frac{1}{5} \gamma_{6} & 0 & \frac{1}{3} \eta \gamma_{6} / n \\ \frac{1}{15} R \gamma_{3} & \frac{1}{10} R \gamma_{3} & -\frac{2}{3} R \gamma_{4} & \frac{1}{10} \gamma_{3} & \frac{2}{3} \gamma_{4} & \frac{1}{6} \eta \gamma_{3} / n \\ -\frac{1}{5} R & -\frac{7}{15} R & 0 & \frac{1}{5} & 0 & \frac{1}{3} \eta / n \\ \frac{2}{5} R \gamma_{5} & \frac{3}{5} R \gamma_{5} & -\frac{1}{3} R & \frac{3}{5} \gamma_{5} & \frac{1}{3} & -\eta \gamma_{5} / n \\ 0 & 0 & 0 & 0 & 0 & 0\end{array}\right]$

Inversion of $\mathbb{1}-\mathbf{A}^{c} \mathbf{B}^{c}$ produces, up to corrections of relative order $l / R$, $\left(1-\mathbf{A}^{c} \mathbf{B}^{c}\right)^{-1}$

$$
=\frac{1}{D}\left[\begin{array}{cccccc}
\frac{(7 u-3) s}{5} & \frac{-2 R \gamma_{1} t}{15} & \frac{R \gamma_{2}(7 u-3)}{15} & \frac{-2 \gamma_{1} t(2 u-1)}{5} & \frac{-\gamma_{2}(7 u-3)}{15} & \frac{-2 \eta \eta_{1} t(2 u-1)}{3 n} \\
\frac{-3(u-1) s}{5} & \frac{2 R \gamma_{1} t}{15} & \frac{-R \gamma_{2}(u-1)}{5} & \frac{2 \gamma_{1} t(u-1)}{5} & \frac{\gamma_{2}(u-1)}{5} & \frac{2 \eta \gamma_{1} t(u-1)}{3 n} \\
\frac{R \gamma_{3} u\left(1-\frac{1}{3} r\right)}{30} & 0 & \frac{2 R \gamma_{1}\left(1-\frac{1}{3} q\right) u}{15} & 0 & \frac{R \gamma_{1} p u}{90} & 0 \\
\frac{-R s}{5} & \frac{-2 R^{2} \gamma_{1} t}{45} & \frac{-R^{2} \gamma_{2}}{15} & \frac{2 R \gamma_{1} t(u+1)}{15} & \frac{R \gamma_{2}}{15} & \frac{2 \eta R \gamma_{1} t}{9 n} \\
\frac{R \gamma_{3} u\left(2 \gamma_{5}-R r\right)}{90} & 0 & \frac{-2 R^{2} \gamma_{1} g u}{45} & 0 & \frac{R \gamma_{1}(12+R p) u}{90} & 0 \\
0 & 0 & 0 & 0 & 0 & 0
\end{array}\right](7
$$


with the abbreviations

$$
\begin{gathered}
p=8 \gamma_{4}-\left(\gamma_{2} \gamma_{3} / \gamma_{1}\right), \quad q=1-\frac{3}{2}\left(\gamma_{2} \gamma_{5} / \gamma_{1}\right) \\
r=1-12\left(\gamma_{5} \gamma_{4} / \gamma_{3}\right), \quad s=\left(1+\frac{2}{3} R \gamma_{4}-\frac{1}{3}\right), \quad t=1+\frac{1}{12} R p-\frac{1}{3} q \\
u=1+\frac{1}{3} R \gamma_{6}, \quad D=\operatorname{Det}\left(1-\mathbf{A}^{c} \mathbf{B}^{c}\right)=\frac{2}{15} R \gamma_{1} t u
\end{gathered}
$$

Equation (7.21) can now be solved, with the result

$$
\begin{gathered}
S_{\mathrm{II}}^{c}=\frac{3}{2} \frac{\eta V}{R\left(3+R \gamma_{6}\right)}\left(\begin{array}{c}
6+R \gamma_{6} \\
R \gamma_{6} \\
0 \\
R \\
0 \\
0
\end{array}\right) \\
S_{\mathrm{II}}^{\mathrm{c}}=S_{\mathrm{II}}^{0, c}[1+O(l / R)]
\end{gathered}
$$

The resulting distribution function follows from (7.20) as

$$
\hat{\Psi}_{\mathrm{NS}}=\frac{V}{u_{0}}\left(\frac{3+\frac{3}{2} R \gamma_{6}}{3+R \gamma_{6}} \hat{\Psi}_{1}+\frac{\frac{1}{4} R \gamma_{6}}{3+R \gamma_{6}} \hat{\Psi}_{2}-\hat{\Psi}_{4}\right)
$$

This expression agrees with Eq. (6.40) in the limit $z \rightarrow 0$. It therefore follows that the drag force on the sphere will agree with Eq. (6.43d) when the limit $z \rightarrow 0$ is taken. It seems certain that a similar continuum calculation as that given here but with $z \neq 0$ will lead to the full result, Eq. (6.43d), but we have not checked this.

We conclude this section with a few comments.

(a) One should notice that the final source $S_{I}^{c}$ [Eq. (7.24)] agrees with $S_{I}{ }^{*}$ given by Eqs. (6.35) and (6.36) in the limit of $z \rightarrow 0$, except for the presence of an amount of $S_{4}{ }^{c}$ in $S_{\mathrm{I}}{ }^{c}$. In spite of the fact that the sources have been defined in different ways in Sections 6 and 7 , the identity of the final expressions for $S_{\mathrm{I}}^{c}$ and $S_{\mathrm{I}}^{*}$ can be understood by noting that the determination of the radial currents of momentum, particles, and energy also fixes the sources of these currents at the surface of the sphere.

(b) The occurrence of $S_{4}{ }^{c}$ in $S_{1}{ }^{c}$ can be understood by noticing that the sources $S_{1}{ }^{c}$ and $S_{2}{ }^{c}$ generate certain amounts of $\hat{\Psi}_{5}$ as well as of $\hat{\Psi}_{1}$ and $\hat{\Psi}_{2}$. Since $\hat{\Psi}_{5}$ does not vanish in the interior of the sphere, the total amount of $\hat{\Psi}_{5}$ generated by all the sources should be zero. In Section 6 we ignored all amounts of $\hat{\Psi}_{5}$ generated by any of the sources, since if the distribution function vanishes in the interior of the sphere, as it should, the amounts of $\hat{\Psi}_{5}$ should eventually cancel, and one does not need to take $\hat{\Psi}_{5}$ into account in the calculation of any quantity of physical interest. Now in this section 
we were able to explicitly determine the amounts of $\hat{\Psi}_{5}$ generated by the sources and hence could show that the total amount of $\hat{\Psi}_{5}$ thus generated vanishes. The source $S_{4}{ }^{c}$, which is of a gradient type, generates exclusively a distribution function $\hat{\Psi}_{5}$, and in $S_{1}^{c}$, the source $S_{4}{ }^{c}$ occurs in precisely the right way to cancel the amount of $\hat{\Psi}_{5}$ generated by $S_{1}{ }^{c}$ and $S_{2}{ }^{c} \cdot{ }^{13}$

(c) From remark (b) one can understand why the functions $\hat{\Psi}_{y}$ were expanded to first order in $l / R$ in the derivation of Eq. (7.4). The terms first order in $l / R$ are responsible for the gradient terms on the right-hand side of Eq. (7.4), and the source term $S_{4}$ is contained in these gradient terms alone.

(d) The procedure adopted in this section provides a justification for the method employed in Section 6. Here we could explicitly determine the amount of $\hat{\Psi}_{5}$ and $\hat{\Psi}_{6}$ generated by the sources, and we could show that the results obtained by taking these terms into account are identical to those obtained when $\hat{\Psi}_{5}$ and $\hat{\Psi}_{6}$ are systematically ignored.

(e) There is also a difference between this section and Section 6 in the way that the source $S_{\mathrm{II}}^{0}$ is dealt with. Namely, in Section 6 we observed that $S_{\mathrm{II}}^{0}$ is the exact source of the inside hydrodynamic solution $-\left[V(z) / u_{0}\right] \hat{\Psi}_{4}$, Eq. (6.27b). In this section we used the projection operator to express $S_{\mathrm{II}}^{0, c}$ in terms of $S_{1}{ }^{\mathrm{c}}, S_{2}{ }^{\mathrm{c}}, S_{4}{ }^{\mathrm{c}}$, and $S_{4}^{\prime c}$. Explicit calculation shows that the distribution function generated by $S_{I I}^{0, c}$ is identical to $-\left[V(z=0) / u_{0}\right] \hat{\Psi}_{4}(z=0)$.

(f) The identity of $S_{\mathrm{II}}$ and $S_{\mathrm{II}}^{0}$ is a result of the fact that $S_{\mathrm{II}}$ is a zero eigenvector of $\mathbf{A B}$. The corrections of order $l / R$ in (7.22b) arise from the neglect of similar corrections in (7.21). The reason that $S_{\mathrm{II}}$ is a zero eigenfunction of $\mathbf{A B}$ is that it generates only a distribution function inside the sphere. $\overline{\mathbf{T}}$ acting on this distribution function then produces zero.

\section{DISCUSSION}

Let us briefly summarize the content of the preceding sections. We started from an extended Boltzmann equation describing a dilute gas at rest at infinity in which a macroscopic sphere performs an arbitrary translational motion in the $z$ direction. Compared to the ordinary Boltzmann equation, this equation contains an extra term describing changes in the distribution function due to collisions of gas molecules with the sphere. The ChapmanEnskog solution of the ordinary Boltzmann equation turned out in general not to be a solution of the extended Boltzmann equation, because the interaction between gas particles and sphere requires that the distribution function

${ }^{13}$ It is worth mentioning that $S_{4}{ }^{c}$ must be a constant multiple of the source $S_{5}$ discussed in Section 6. Similarly, $S_{1}{ }^{c}$ must be equal to $S_{1}$ plus some amount of $S_{5}$. 
satisfies certain boundary conditions at the surface of the sphere which are not satisfied by the Chapman-Enskog solution.

In Section 3, we argued that the actual solution of the extended Boltzmann equation must consist of two parts, one part which has the Chapman-Enskog form and the other part which describes a kinetic boundary layer extending over a few mean free paths around the sphere. The two parts cannot be independently determined, but instead are related to each other by the boundary conditions that the distribution function satisfies at the surface of the sphere. As a result, any systematic description of the distribution function must take the effects of the boundary layer into account. In the remainder of the paper we have shown how this can be done. We introduced projection operators which allowed us to decompose the distribution function into a Chapman-Enskog, or hydrodynamic, part and a boundary layer part. In this way we could obtain a formal expression for the hydrodynamic part of the distribution function where the effects of the boundary layer are explicitly taken into account. We also obtained a formal expression for the boundary layer part of the distribution function. We then showed how one could systematically derive various properties, at least to lowest order in $l / R$, without having to ignore the presence of the kinetic boundary layer. In particular, we gave a derivation of Stokes' law for the force on the sphere. Although this result is hardly new, our derivation is the first one from the Boltzmann equation which incorporates the effect of the boundary layer and does not depend on some specific model for the gas molecules. It is, however, very intricate.

Part of the difficulties encountered in this derivation revolved around the construction of the proper projection operator to be used in the decomposition of the distribution function into its hydrodynamic and boundary layer parts. We first introduced a projection operator which seemed to be a natural one, namely the projection onto the space of the usual hydrodynamic modes.

It then turned out that this projection operator produced a rather unphysical description of the normal solution close to the boundary of the sphere. This could be repaired by passing to a projection operator projecting onto the space of hydrodynamic solutions which are discontinuous at the surface of the sphere.

Although we could not construct the operator explicitly, we were still able to determine its action on a distribution function or a source term to leading order in $l / R$ : Thereto we used the requirement that to lowest order in the Knudsen number the radial components of the currents of mass, momentum, and energy are given everywhere by the hydrodynamic part of the distribution function alone. This then enabled us to solve the extended Boltzmann equation to leading order in $l / R$ and to obtain the hydrodynamic 
part of the distribution function and the drag force on the sphere to this same order. In the continuum limit finally, where the kinetic boundary layer shrinks onto the boundary, the objections against the usual hydrodynamic projection operator are no longer important. The action of the latter was worked out explicitly in Section 7 and found to lead to the same results for the hydrodynamic distribution function and the force on the sphere as was obtained in Section 6. However, the method of Section 6 provides a decomposition of the distribution function which is physically more satisfactory if one does not pass to the continuum limit.

The method we used differs from more conventional methods in that it calculates the distribution function directly and not by supplementing a set of differential equations (the hydrodynamic equations) with boundary conditions at the surface of the sphere. Yet the resulting fluid fields do satisfy these boundary conditions as expected, at least to lowest order in $l / R$. A refinement of the boundary conditions to the next order in $l / R$ cannot be obtained unless one can calculate the kinetic boundary layer.

It is interesting to compare the source terms in our solution with those used by Scharf. ${ }^{(10)}$ Scharf considers only the case of stick boundary conditions, for which he has a source $-\frac{3}{2}(\eta / R)(\mathbf{v} \cdot \hat{\mathbf{r}})(\mathbf{v} \cdot \mathbf{V}) \delta(r-R)$. This is precisely in agreement with our results (7.24a), (7.8), and (7.6) for the total source of the distribution function to leading order in $l / R$. However, Scharf simply postulates this source term and does not indicate how it could result from the microscopic interaction mechanism between gas molecules and the sphere.

In the theory of rarefied gas dynamics the drag force is usually calculated, as mentioned in the introduction, by summing an infinite series of terms ${ }^{(2,13)}$ in which the $n$th term contains the contributions of all dynamical events in which $n$ correlated collisions between a gas molecule and the sphere occur. $^{(2,13)}$ It is very interesting that the same method can also be used in the hydrodynamic regime. One then obtains a divergent series which can be identified with an iteration of (6.34b) in powers of $\mathbf{A B}$. Resummation of this series brings one back to (6.43e) and the well-known hydrodynamic solution is obtained again. The details of this analysis will be discussed in a subsequent paper, where we will also discuss the refinements needed in the case of the drag on an infinitely long cylinder moving in a direction perpendicular to its axis.

\section{APPENDIX A. SOLUTIONS OF THE LINEARIZED HYDRODYNAMIC EQUATIONS}

Our starting point is furnished by the linearized hydrodynamic equations for a dilute gas, ${ }^{(2-7)}$

$$
z \delta n / n+\nabla \cdot \mathbf{u}=0
$$




$$
\begin{aligned}
z \mathbf{u} & =-(1 / m \beta) \nabla(\delta n / n+\delta T / T)+v \nabla^{2} \mathbf{u}+\frac{1}{3} v \nabla(\nabla \cdot \mathbf{u}) \\
z \delta T / T & =-\frac{2}{3}(\nabla \cdot \mathbf{u})+\frac{5}{3} D_{T} \nabla^{2} \delta T / T
\end{aligned}
$$

First we recall that the gradient is of order $R^{-1}$ and, on the time scale in which we are interested, $z$ is of order $v / R^{2}$. Then it follows from (A.1) and (A.2) that

$$
\begin{aligned}
\frac{\delta n}{n}+\frac{\delta T}{T} & =O\left[\frac{l}{R}(\beta m)^{1 / 2}|u|\right] \\
\nabla \cdot \mathbf{u} & =O\left[\frac{l}{R} \frac{1}{(\beta m)^{1 / 2} R} \frac{\delta n}{n}\right]
\end{aligned}
$$

To be consistent with our truncation of the linearized extended Boltzmann equation at $O(\nabla)$ we must calculate the flow fields to leading and first order in $l / R$ only. This means, for instance, that we must set $\nabla \cdot \mathbf{u}=0$ in case $\delta n / n=O(l / R)$.

One set of solutions of (A.1)-(A.3) is obtained by setting $\delta T / T=O(1)$. Equations (A.1) and (A.4) then lead to

$$
\begin{aligned}
& \delta n / n=-\delta T / T+O(l / R) \\
& \nabla \cdot \mathbf{u}=z \delta T / T+O(l / R)^{3}
\end{aligned}
$$

Substitution of (A.7) into (A.3) yields

$$
z \delta T / T=D_{T} \nabla^{2}(\delta T / T)
$$

The general solution of this equation can be given in the form of an expansion in products of spherical harmonics and spherical Bessel functions of imaginary argument as ${ }^{(22)}$

$$
\begin{aligned}
\delta T / T & =\sum_{m, l} C_{m l} Y_{l}^{m}(\hat{\mathbf{r}}) k_{l}(r / \sigma), & & r>R \\
& =\sum_{m, l} D_{m l} Y_{l}^{m}(\hat{\mathbf{r}}) i_{l}(r / \sigma), & & r<R
\end{aligned}
$$

The functions $k_{l}$ and $i_{l}$ can be expressed in terms of ordinary Bessel functions of imaginary argument as

$$
k_{l}(x)=(\pi / 2 x)^{1 / 2} K_{l+1 / 2}(x), \quad i_{l}(x)=(\pi / 2 x)^{1 / 2} I_{l+1 / 2}(x)
$$

In our case the only spherical harmonic excited is $Y_{1}{ }^{0}$ (the $z$ axis is chosen along $\mathbf{V}$ ), so that $\delta T / T$ is of the form

$$
\frac{\delta T}{T}=C_{1} e^{-r / \sigma}\left(\frac{\sigma^{2}}{r^{2}}+\frac{\sigma}{r}\right)(\hat{\mathbf{z}} \cdot \hat{\mathbf{r}}), \quad r>R
$$




$$
=D_{1}\left[e^{r / \sigma}\left(-\frac{\sigma^{2}}{r^{2}}+\frac{\sigma}{r}\right)+e^{-r / \sigma}\left(\frac{\sigma^{2}}{r^{2}}+\frac{\sigma}{r}\right)\right](\hat{\mathbf{z}} \cdot \hat{\mathbf{r}}), \quad r<R
$$

The corresponding irrotational velocity [which is of $O(l / R)$ ] is obtained from (A.7).

Another set of solutions of (A.1)-(A.3) is obtained by setting $\delta T / T=0$. From (A.6) and (A.7) it follows then that $\nabla \cdot \mathbf{u}=0$ through relevant orders in $l / R$. Hence we can set

$$
\mathbf{u}=\boldsymbol{\nabla} \times \mathbf{A}
$$

and require, without loss of generality,

$$
\boldsymbol{\nabla} \cdot \mathbf{A}=\mathbf{0}
$$

Taking the curl of (A.2), one then obtains

$$
\left(z-v \nabla^{2}\right) \nabla^{2} \mathbf{A}=0
$$

For simplicity we restrict ourselves to solutions which have rotational symmetry about the $z$ axis. We can separate $A$ into: (1) a radial field, orthogonal to the $z$ axis, (2) a parallel field, parallel to the $z$ axis, and (3) a toroidal field, orthogonal to the first two fields. All three fields may be assumed to possess rotational symmetry about the $z$ axis. In that case both the radial and the parallel fields give rise to a toroidal velocity field, which does not interest us here. Hence only the toroidal field remains. According to (A.13), it has to satisfy either $\nabla^{2} \mathbf{A}=\mathbf{0}$ or $\left(z-v \nabla^{2}\right) \mathbf{A}=0$. The required solutions of these equations in spherical coordinates, with $x=r \sin \Theta \cos \varphi$ $y=r \sin \Theta \sin \varphi$, and $z=r \cos \Theta$, can be expanded generally as ${ }^{(22)}$

$$
\left.\begin{array}{ll}
A_{x}^{(1)}=-\sum_{l} \frac{c_{l}}{r^{l+1}} P_{l}^{1}(\cos \Theta) \sin \varphi \\
A_{y}^{(1)}=\sum_{l} \frac{c_{l}}{r^{l+1}} P_{l}^{1}(\cos \Theta) \cos \varphi
\end{array}\right\} \quad r>R
$$




$$
\left.\begin{array}{l}
A_{x}^{(2)}=-\sum_{l} d_{l}^{\prime} i_{l}(r / \rho) P_{l}{ }^{1}(\cos \Theta) \sin \varphi \\
A_{y}^{(2)}=\sum_{l} d_{l}^{\prime} i_{l}(r / \rho) P_{l}{ }^{1}(\cos \Theta) \cos \varphi
\end{array}\right\} \quad r<R
$$

where $P_{l}^{m}$ denotes the Legendre polynomials.

From (A.11) the corresponding velocity fields are obtained as

$$
\begin{aligned}
& \left.\begin{array}{l}
u_{r}^{(1)}=-\sum_{l} c_{l} \frac{l(l+1)}{r^{l+2}} P_{l}^{0}(\cos \Theta) \\
u_{\Theta}^{(1)}=-\sum_{l} c_{l} \frac{l}{r^{l+2}} P_{l}{ }^{1}(\cos \Theta)
\end{array}\right\} \quad r>R \\
& \left.\begin{array}{l}
u_{\mathrm{r}}^{(1)}=-\sum_{l} c_{l}^{\prime} l(l+1) r^{l-1} P_{l}^{0}(\cos \Theta) \\
u_{\Theta}^{(1)}=\sum_{l} c_{l}^{\prime}(l+1) r^{l-1} P_{l}^{1}(\cos \Theta)
\end{array}\right\} \quad \begin{array}{r}
r<R \\
\quad
\end{array} \\
& \left.\begin{array}{l}
u_{\mathbf{r}}^{(2)}=-\sum_{l} d_{l} l(l+1) \frac{\rho}{r} k_{l}\left(\frac{r}{\rho}\right) P_{l}^{0}(\cos \Theta) \\
u_{\Theta}^{(2)}=\sum_{l} d_{l}\left(\frac{\rho}{r}+\rho \frac{d}{d r}\right) k_{l}\left(\frac{r}{\rho}\right) P_{l}{ }^{1}(\cos \Theta)
\end{array}\right\} r>R \\
& \left.\begin{array}{l}
u_{r}^{(2)}=-\sum_{l} d_{l}^{\prime} l(l+1) \frac{\rho}{r} i_{l}\left(\frac{r}{\rho}\right) P_{l}^{0}(\cos \Theta) \\
u_{\Theta}^{(2)}=\sum_{l} d_{l}^{\prime}\left(\frac{\rho}{r}+\rho \frac{d}{d r}\right) i_{l}\left(\frac{r}{\rho}\right) P_{l}{ }^{1}(\cos \Theta)
\end{array}\right\} r<R
\end{aligned}
$$

The corresponding density fields are determined as follows: From (A.2) and $(\nabla \cdot \mathbf{u})=0$ one obtains $\nabla^{2} \delta n / n=0$. The general solution with rotational symmetry about the $z$ axis reads:

$$
\begin{aligned}
\delta n / n & =\sum_{l} A_{l} r^{-(l+1)} P_{l}^{0}(\cos \Theta), & & r>R \\
& =\sum_{l} A_{l}{ }^{\prime} r^{l} P_{l}^{0}(\cos \Theta), & & r<R
\end{aligned}
$$

From which one obtains for the gradients of $\delta n / n$

$$
\begin{aligned}
\nabla(\delta n / n)_{r} & =-\sum_{l} A_{l}(l+1) r^{-(l+2)} P_{l}^{0}(\cos \Theta) & & r>R \\
& =\sum_{l} A_{l}{ }^{\prime} l r^{l-1} P_{l}^{0}(\cos \Theta) & & r<R
\end{aligned}
$$




$$
\begin{aligned}
\nabla(\delta n / n)_{\Theta} & =-\sum_{l} A_{l} r^{-(l+2)} P_{l}^{1}(\cos \Theta) & & r>R \\
& =-\sum_{l} A_{l}{ }^{\prime} r^{l-1} P_{l}{ }^{1}(\cos \Theta) & & r<R
\end{aligned}
$$

The coefficients $A_{l}$ and $A_{l}^{\prime}$ are obtained by identification with (A.2); $\nabla(\delta n / n)=-\beta m n\left(z-v \nabla^{2}\right) \mathbf{u}$. The field $\mathbf{u}^{(1)}$ satisfies $\nabla^{2} \mathbf{u}^{(1)}=0$; hence

$$
\nabla\left(\delta n^{(1)} / n\right)=-\beta m n z \mathbf{u}^{(1)}=-\left(\beta \eta / \rho^{2}\right) \mathbf{u}^{(1)}
$$

and one finds

$$
\begin{aligned}
\frac{\delta n^{(1)}}{n} & =-\sum_{l} \frac{\beta \eta l}{\rho^{2}} c_{l} r^{-(l+1)} P_{l}^{0}(\cos \Theta) & & r>R \\
& =-\sum_{l} \frac{\beta \eta}{\rho^{2}}(l+1) c_{l}{ }^{\prime}{ }^{l} P_{l}{ }^{0}(\cos \Theta) & & r<R
\end{aligned}
$$

The field $\mathbf{u}^{(2)}$ satisfies $\left(z-v \nabla^{2}\right) \mathbf{u}^{(2)}=0$; hence $\delta n^{(2)} / n=0$.

Specification to $l=1$ leads to $\hat{\Phi}_{1}, \hat{\Phi}_{2}, \hat{\Phi}_{4}$, and $\hat{\Phi}_{5}$ as given in (5.3)

\section{APPENDIX B. RADIAL CURRENTS IN THE BOUNDARY LAYER}

In this appendix we want to demonstrate why even in the boundary layer the radial currents of momentum, energy, and particles are determined, to leading order in the Knudsen number, entirely by the hydrodynamic part of the distribution function.

To show this we start by considering stationary flow and look at the distribution function $\Psi$ generated by a source $S \delta(r-R)$, with $S$ of order unity in $l / R$. As usual $\Psi$ may be decomposed into $\Psi=P \Psi+P_{\perp} \Psi$, where in the stationary case $P \Psi$ and $P_{\perp} \Psi$ are given respectively by

$$
P \Psi=[P(\mathbf{v} \cdot \nabla-L) P]^{-1} P S \delta(r-R)
$$

and

$$
P_{\perp} \Psi=\left[P_{\perp}(\mathbf{v} \cdot \nabla-L) P_{\perp}\right]^{-1} P_{\perp} S \delta(r-R)
$$

One can argue that as a function of $l / R, P_{\perp} \Psi$ has the same magnitude as $P_{\perp} S$. To see this, we first use the fact that only the sources within a region of a few mean free paths in diameter can contribute to $P_{\perp} \Psi$ at any given point. Then in the limit as $l / R \rightarrow 0$, the surface reduces to a flat plate with a uniform source density and $P_{\perp} \Psi$ approaches a finite limit which is proportional to the source $P_{\perp} S$. Now since $P_{\perp} \Psi$ is finite and proportional to $P_{\perp} S$, and since $l / R=0$, it must be true that $P_{\perp} \Psi$ and $P_{\perp} S$ have the same magnitude in orders of $l / R$. 
Next we show that the radial currents generated by $P_{\perp} \Psi$, namely

$$
j_{i, r}^{\perp}=\hat{\mathbf{r}} \cdot \mathbf{j}_{i}{ }^{\perp}=\left\langle(\mathbf{v} \cdot \hat{\mathbf{r}}) n_{i} P_{\perp} \Psi\right\rangle
$$

with $n_{i}$ defined by Eq. (6.4), are of order $l / R$ compared to $P_{\perp} \Psi$. This can be seen by observing that, for example, $\nabla \cdot \mathbf{j}_{3}{ }^{1}=0$, which in spherical coordinates reads

$$
r^{-2}(\partial / \partial r)\left(r^{2} j_{3}^{\perp}, r\right)+(r \sin \Theta)^{-1}(\partial / \partial \Theta)\left[(\sin \Theta) j_{3, \Theta}^{\perp}\right]=0
$$

where we have set $j_{3, \varphi}^{\perp}=0$. Now we use the fact that $\mathbf{j}_{3}{ }_{3}^{\perp}=0$ beyond a few mean free paths from the sphere, and integrate $(\partial / \partial r)\left(r^{2} j_{3, r}^{\perp}\right)$ along a radius from $R$ to $R+\alpha l$. Then we find that

$$
j_{3, r}^{\perp}(R)=-\frac{1}{R^{2}} \int_{R+\alpha t}^{R} d r \frac{r}{\sin \Theta} \frac{\partial}{\partial \Theta}\left[(\sin \Theta) j_{3, \Theta}^{\perp}\right]
$$

Then, since $j_{3}^{\frac{1}{3}}, \Theta$ can be at most of $O(1)$, we conclude that $\dot{j}_{3, r}$. can be at most of $O(l / R)$. A similar discussion can be given for the other radial currents. However, the radial currents generated by the source $\delta(r-R) S$ are in general of $O(1)$. This implies that to order unity the radial currents are completely determined by $P \Psi$, which is what we wanted to show, at least for the stationary case.

It is worth pointing out that these arguments also lead to the conclusion that if $P S$ is of order unity in $l / R$, then $P \Psi$ is in general of relative order $R / l$. To see this simply compute the radial currents of energy and momentum using $P \Psi$. The first terms in $P \Psi$ which give nonvanishing contributions to these currents are terms involving $(\delta n / n+\delta T / T)$ as well as the gradients of the velocity and temperature fields. All of these are of relative order $l / R$, while the radial current are of order 1 , so that $P \Psi$ must be of order $R / l$.

Finally, we must consider what happens in the nonstationary case. In this case we allow only for variations on a time scale $t^{\text {hd }}=R^{2} / v \sim\left(R^{2} / l^{2}\right) t^{\mathrm{mf}}$, where $t^{\mathrm{mf}}$ is the mean free time between collisions. The nonhydrodynamic solutions generated by a source at the surface of the sphere decay to zero in a few mean free times, and can therefore be written as

$$
P_{\perp} \Psi(t)=\int_{0}^{t_{0}} d \tau \exp \left[P_{\perp}(\mathbf{v} \cdot \nabla-L) P_{\perp} \tau\right] P_{\perp} S(t-\tau) \delta(r-R)
$$

For $t_{0}$ one may choose $t_{0}=10 t^{m f}$, for example, because then the nonhydrodynamic solutions generated by sources at times before $\tau=t-t_{0}$ will have died off at time $t$. Equation (B.4) can be rewritten as

$P_{\perp} \Psi(t)=\int_{0}^{t_{0}} d \tau \exp \left[P_{\perp}(\mathbf{v} \cdot \nabla-L) P_{\perp} \tau\right] P_{\perp}\left[S_{0}+\Delta S(t-\tau)\right] \delta(r-R)$ 
with

$$
S_{0}=\frac{1}{t_{0}} \int_{0}^{t_{0}} d \tau S(t-\tau) ; \quad \Delta S=S-S_{0}
$$

The source $S_{0}$ produces a stationary solution of the linearized Boltzmann equation, which has radial currents of relative order $l / R$, and the source $\Delta S$ is of the order $(l / R)^{2} S_{0}$ due to the slowness with which the source varies. Hence the extra contributions to the radial currents in the nonhydrodynamic solution which are due to nonstationarity are at most of order $(l / R)^{2}$. Thus to leading order the radial currents are still determined by the hydrodynamic part of the distribution function.

\section{APPENDIX C. THE ACTION OF P華 ON A NORMAL SOLUTION OF THE BOLTZMANN EQUATION}

In order to obtain the action of $P \tilde{\overline{\mathbf{T}}}$ we start from the action of $\overline{\mathbf{T}}$ acting on $f_{\mathrm{NS}}$. This is obtained by linearization of (3.10) and (3.12) and use of (2.15) as

$$
\begin{aligned}
\overline{\mathbf{T}}_{\mathrm{di}} \hat{\Psi}_{\mathrm{NS}}= & (\mathbf{v} \cdot \hat{\mathbf{r}}) \delta(r-R)\left\{\Theta ( \mathbf { v } \cdot \hat { \mathbf { r } } ) \left[-(\pi \beta m / 2)^{1 / 2}(\mathbf{u} \cdot \hat{\mathbf{r}})+(\delta n / n+\delta T / T)\right.\right. \\
& \left.-\frac{1}{2} \delta T / T+\frac{1}{2} C_{1}(3 \hat{\mathbf{r}} \hat{\mathbf{r}}-1): \mathbf{V u}\right]+\Theta(-\mathbf{v} \cdot \hat{\mathbf{r}}) \\
& \times\left[\beta m(\mathbf{v} \cdot \mathbf{u})+(\delta n / n+\delta T / T)+(\delta T / 2 T)\left(\beta m v^{2}-5\right)\right. \\
& \left.\left.+B\left(v^{2}\right)\left(\mathbf{v v}-\frac{1}{3} v^{2} 1\right): \nabla \mathbf{u}+A\left(v^{2}\right)(\mathbf{v} \cdot \mathbf{v})(\delta T / T)\right]+O(l / R)^{2}\right\} \\
\overline{\mathbf{T}}_{\alpha} \hat{\Psi}_{\mathrm{NS}}= & (\mathbf{v} \cdot \hat{\mathbf{r}}) \delta(r-R)\{\Theta(\mathbf{v} \cdot \hat{\mathbf{r}})[(1-\alpha) \beta m[(\mathbf{v} \cdot \mathbf{u})-2(\mathbf{v} \cdot \hat{\mathbf{r}})(\mathbf{u} \cdot \hat{\mathbf{r}})] \\
& -\alpha(\pi \beta m / 2)^{1 / 2}(\mathbf{u} \cdot \hat{\mathbf{r}})+(\delta n / n+\delta T / T) \\
& +(\delta T / 2 T)\left[(1-\alpha) \beta m v^{2}-5(1-\alpha)-\alpha\right] \\
& +B\left(v^{2}\right)\left\{[\mathbf{v}-2(\mathbf{v} \cdot \hat{\mathbf{r}}) \hat{\mathbf{r}}][\mathbf{v}-2(\mathbf{v} \cdot \hat{\mathbf{r}}) \hat{\mathbf{r}}]-\frac{1}{3} v^{2 \vartheta\}}: \nabla \mathbf{u}\right. \\
& \left.+A\left(v^{2}\right)[\mathbf{v}-2(\mathbf{v} \cdot \hat{\mathbf{r}}) \hat{\mathbf{r}}] \cdot \nabla(\delta T / T)\right]+\Theta(-\mathbf{v} \cdot \hat{\mathbf{r}}) \\
& \times\left[\beta m(\mathbf{v} \cdot \mathbf{u})+(\delta n / n+\delta T / T)+(\delta T / 2 T)\left(\beta m v^{2}-5\right)\right. \\
& \left.\left.+B\left(v^{2}\right)\left(\mathbf{v v}-\frac{1}{3} v^{2} 0\right): \nabla \mathbf{u}+A\left(v^{2}\right)(\mathbf{v} \cdot \nabla)(\delta T / T)\right]+O(l / R)^{2}\right\}
\end{aligned}
$$

In Eq. (C.1), the quantity $C_{1}$ is a constant. Here we used that $\alpha \sim l / R$ and that terms containing $\nabla \mathbf{u}$ and $\nabla \delta T$ are of order $l / R$ compared to those containing $\mathbf{u}$ and $\delta T$, respectively. Next one must let the boundary layer propagator $\left[P_{\perp}(\mathbf{v} \cdot \nabla-L-\overline{\mathbf{T}}) P_{\perp}\right]^{-1}$ act upon this. To leading order in $l / R$ the $\mathbf{v} \cdot \nabla$ occurring in here reduces to $v_{r} \partial / \partial r$, since the typical length for the gradient in this case is the mean free path and on this length scale the sphere appears as a flat plate to lowest order. This implies that 
to leading order in $l / R$, the propagator leaves the functional forms $\cos \Theta f\left(v_{r}, v^{2}, v_{\Theta}^{2}, r\right)$ and $\sin \Theta v_{\Theta} f\left(v_{r}, v^{2}, v_{\Theta}{ }^{2}, r\right)$ unchanged.

Let us now first consider $\overline{\mathbf{T}}_{\mathrm{di}} \Psi_{\mathrm{NS}}$. The contributions to the sources $S_{1}, S_{2}$, and $S_{3}$ from the terms in (C.1) containing gradients of $\mathbf{u}$ or $\delta T$ are always of order $l / R$ compared to contributions from terms without gradients and hence can be neglected. The hydrodynamic solutions we consider are always linear combinations of $\hat{\Psi}_{1}, \hat{\Psi}_{2}, \hat{\Psi}_{3}$, and $\hat{\Psi}_{4}$ given in (6.5). For $\hat{\Psi}_{3}$ one has $\delta n / n+\delta T / T=0$, for $\hat{\Psi}_{1}$ and $\hat{\Psi}_{2}, \delta T / T=0$ and $\delta n / n \sim$ $(l / R)(2 \pi \beta m)^{1 / 2}(\mathbf{u} \cdot \hat{\mathbf{r}}) ;$ hence $\delta n / n+\delta T / T$ can always be neglected when one determines hydrodynamic sources from $\overline{\mathbf{T}}_{\mathrm{di}} \hat{\mathbf{T}}_{\mathrm{Ns}}$. Next we consider the complete operator $\tilde{\widetilde{T}}$. By considering the tensorial character of the radial currents, we can see that to leading order in $l / R$ the operator $\tilde{\widetilde{T}}$ couples the orthogonal momentum source and the energy source to the radial velocity and the temperature jump at the surface of the sphere, and it couples the tangential momentum source to the tangential velocity at the surface. This leads to (6.14) in the case that $\overline{\mathbf{T}}=\overline{\mathbf{T}}_{\mathrm{di}}$.

Consider next $\overline{\mathbf{T}}_{\alpha}$. To leading order in $l / R$ this operator couples the orthogonal momentum current to the orthogonal velocity at the surface. The operator $\overline{\mathbf{T}}_{\alpha}$ does not couple the radial momentum current to the temperature jump at the surface of the sphere to leading order in $l / R$, since the integral $\left\langle(\mathbf{v} \cdot \hat{\mathbf{r}}) \overline{\mathbf{T}}_{\mathrm{sp}}\left(\frac{1}{2} \beta m v^{2}-\frac{5}{2}\right)\right\rangle$ vanishes. $J_{2}$ and $J_{3}$, the tangential momentum current and the energy current, are left eigenfunctions of $\overline{\mathbf{T}}_{\mathrm{sp}}$, which implies that for $\overline{\mathbf{T}}_{\alpha} \hat{\Psi}_{\text {NS }}$ the strengths of $S_{2}$ and $S_{3}$ are of order $l / R$.

When going from $\overline{\mathbf{T}}_{\alpha}$ to $\tilde{\mathbf{T}}_{\alpha}$ we find it useful to use the equality

$$
\begin{aligned}
\overline{\mathbf{T}}_{\alpha} P_{\perp} & {\left[P_{\perp}\left(\mathbf{v} \cdot \nabla-L-\overline{\mathbf{T}}_{\alpha}\right) P_{\perp}\right]^{-1} P_{\perp} \overline{\mathbf{T}}_{\alpha} \hat{\Psi}_{\mathrm{NS}} } \\
& =\overline{\mathbf{T}}_{\alpha} P_{\perp}\left[P_{\perp}\left(\mathbf{v} \cdot \nabla-L-\overline{\mathbf{T}}_{\alpha}\right) P_{\perp}\right]^{-1} P_{\perp} \mathbf{T}_{\alpha} \hat{\Psi}_{\mathrm{NS}}
\end{aligned}
$$

To see this one need only note that

$$
\left(\overline{\mathbf{T}}_{\alpha}-\mathbf{T}_{\alpha}\right) \hat{\Psi}_{\mathrm{NS}}=(\mathbf{v} \cdot \hat{\mathbf{r}}) \delta(r-R) \hat{\Psi}_{\mathrm{NS}}
$$

and that $(\mathbf{v} \cdot \hat{\mathbf{r}}) \delta(r-R) \hat{\Psi}_{\mathrm{NS}}$ is the exact source of the hydrodynamic solution $\hat{\Psi}_{N S}^{*}$, which is equal to $\hat{\Psi}_{\mathrm{NS}}$ outside the sphere and is zero inside the sphere. Hence $P_{\perp}\left(\overline{\mathbf{T}}_{\alpha}-\mathbf{T}_{\alpha}\right) \hat{\Psi}_{\mathrm{NS}}$ vanishes. From Eq. (C.3) we can see that the radial momentum current is still not coupled to the temperature jump at the surface of the sphere to leading order in $l / R$ because $\left(\frac{1}{2} \beta m v^{2}-\frac{5}{2}\right)$ is a right eigenfunction of $\mathbf{T}_{\mathrm{sp}}$ with eigenvalue zero. Furthermore, because $v_{\Theta}$ is a right eigenfunction of $\mathbf{T}_{\mathrm{sp}}$ and a left eigenfunction of $\overline{\mathbf{T}}_{\mathrm{sp}}$ also with eigenvalue zero, it follows that the extra amount of $S_{2}$ produced when going from $\mathbf{T}_{\alpha}$ to $\tilde{\mathbf{T}}_{\alpha}$ is of order $(l / R)^{2}$.

Similarly, the energy source resulting from a temperature jump is determined by $\overline{\mathbf{T}}_{\alpha}$ acting on $(\delta T / 2 T)\left(\beta m v^{2}-5\right)$, but for the energy source resulting 
from a radial velocity field the transition from $\overline{\mathbf{T}}_{\alpha}$ to $\widetilde{\overline{\mathbf{T}}}_{\alpha}$ does alter the source strength, although it remains of order $l / R$. All this results in (6.14) and (6.15).

\section{APPENDIX D. CALCULATION OF THE HYDRODYNAMIC FIELDS GENERATED BY THE SOURCES $\sigma_{1}, \ldots, \sigma_{6}$}

We start with the Fourier-transformed, stationary, linearized NavierStokes equations in the presence of source terms, which follow from (7.6) as

$$
\begin{aligned}
n(i \mathbf{k} \cdot \mathbf{u}) & =\sigma_{n}(\mathbf{k}) \\
i \mathbf{k}\left(\beta^{-1} \delta n+n k_{\mathrm{B}} \delta T\right)+\eta k^{2} \mathbf{u}+\frac{1}{3} \eta(\mathbf{u} \cdot \mathbf{k}) \mathbf{k} & =\sigma_{p}(\mathbf{k}) \\
\lambda k^{2} \delta T & =\sigma_{h}
\end{aligned}
$$

where (D.1c) is obtained by combination of (7.6a) and (7.6c).

The divergence-free velocity field is obtained from (D.lb) as

$$
\mathbf{u}-(\mathbf{u} \cdot \hat{\mathbf{k}}) \hat{\mathbf{k}}=\left(\eta k^{2}\right)^{-1}\left[\boldsymbol{\sigma}_{p}-\left(\boldsymbol{\sigma}_{p} \cdot \hat{\mathbf{k}}\right) \hat{\mathbf{k}}\right]
$$

The irrotational velocity field follows from (D.1a) as

$$
\mathbf{u} \cdot \hat{\mathbf{k}}=(i n k)^{-1} \sigma_{n}
$$

the temperature field from (D.1c) as

$$
\delta T=\left(\lambda k^{2}\right)^{-1} \sigma_{h}
$$

and the density field from (D.1a) and (D.1b) as

$$
\frac{\delta n}{n}+\frac{\delta T}{T}=\frac{\beta}{n}\left(\frac{\sigma_{p} \cdot \hat{\mathbf{k}}}{i k}+\frac{4}{3} \frac{\eta}{n} \sigma_{n}\right)
$$

Consider first the fields resulting from the momentum source (in position language) $\sigma_{1}(r)=(\mathbf{z} \cdot \hat{\mathbf{r}}) \hat{\mathbf{r}} \delta(r-R)$. First the Fourier transform of this source is needed:

$$
\begin{aligned}
\boldsymbol{\sigma}_{1}(\mathbf{k}) & =\int d \mathbf{r}[\exp (-i \mathbf{k} \cdot \mathbf{r})](\mathbf{z} \cdot \hat{\mathbf{r}}) \hat{\mathbf{r}} \delta(r-R) \\
& =\hat{\mathbf{z}} \cdot[A(k) 1+B(k) \hat{\mathbf{k}} \hat{\mathbf{k}}]
\end{aligned}
$$

Equation (D.6b) gives the most general vectorial form possible for $\sigma_{1}(\mathbf{k})$. The functions $A$ and $B$ can be determined by contraction of $\hat{\mathbf{r}} \hat{\mathbf{r}}$ in (D.6a) with 
the unit tensor and the tensor $\hat{\mathbf{k}} \hat{\mathbf{k}}$, respectively, yielding

$$
\begin{aligned}
3 A+B & =\int d \mathbf{r}[\exp (-i \mathbf{k} \cdot \mathbf{r})] \delta(r-R)=4 \pi R^{2} \frac{\sin k R}{k R} \\
A+B & =\int d \mathbf{r}[\exp (-i \mathbf{k} \cdot \mathbf{r})](\mathbf{k} \cdot \hat{\mathbf{r}})^{2} \delta(r-R) \\
& =4 \pi R^{2}\left[\frac{\sin k R}{k R}+\frac{2 \cos k R}{(k R)^{2}}-\frac{2 \sin k R}{(k R)^{3}}\right]
\end{aligned}
$$

or

$$
\begin{aligned}
\sigma_{1}(\mathbf{k})= & 4 \pi R^{2} \hat{\mathbf{z}} \cdot\left\{\left[-\frac{\cos k R}{(k R)^{2}}+\frac{\sin k R}{(k R)^{3}}\right] \mathbb{1}\right. \\
& \left.+\left[\frac{\sin k R}{k R}+\frac{3 \cos k R}{(k R)^{2}}-\frac{3 \sin k R}{(k R)^{3}}\right] \hat{\mathbf{k}} \hat{\mathbf{k}}\right\}
\end{aligned}
$$

From this a divergence-free velocity field is obtained with (D.2) as

$$
\mathbf{u}-(\mathbf{u} \cdot \hat{\mathbf{k}}) \hat{\mathbf{k}}=\frac{4 \pi R^{2}}{\eta k^{2}} \hat{\mathbf{z}} \cdot(1-\hat{\mathbf{k}} \hat{\mathbf{k}})\left[-\frac{\cos k R}{(k R)^{2}}+\frac{\sin k R}{(k R)^{3}}\right]
$$

while the corresponding density field follows from (D.5) as

$$
\delta n=\beta \frac{4 \pi R^{2}}{i k}\left[\frac{\sin k R}{k R}+2 \frac{\cos k R}{(k R)^{2}}-2 \frac{\sin k R}{(k R)^{3}}\right](\hat{\mathbf{z}} \cdot \hat{\mathbf{k}})
$$

The source $\sigma_{1}$ does not contribute to the temperature field and to the irrotational velocity field. The spatial velocity and density field are obtained by an inverse Fourier transform of (D.8a) and (D.8b), viz.

$$
\begin{aligned}
\mathbf{u}(\mathbf{r}) & =\frac{4 \pi R^{2}}{\eta} \frac{1}{(2 \pi)^{3}} \int d \mathbf{k} \frac{\exp (i \mathbf{k} \cdot \mathbf{r})}{k^{2}} \hat{\mathbf{z}} \cdot(\mathbb{1}-\hat{\mathbf{k}} \hat{\mathbf{k}})\left[-\frac{\cos k R}{(k R)^{2}}+\frac{\sin k R}{(k R)^{3}}\right] \\
& =\hat{\mathbf{z}} \cdot[A(r) \mathbb{1}+B(r) \hat{\mathbf{r}} \hat{\mathbf{r}}]
\end{aligned}
$$

Contraction with 1 yields

$$
\begin{aligned}
3 A+B & =\frac{R^{2}}{\eta \pi^{2}} \int d \mathbf{k} \frac{\exp (i \mathbf{k} \cdot \mathbf{r})}{k^{2}}\left[-\frac{\cos k R}{(k R)^{2}}+\frac{\sin k R}{(k R)^{3}}\right] \\
& =\frac{4 R^{2}}{\pi \eta} \int_{0}^{\infty} d k \frac{\sin k r}{k r}\left[-\frac{\cos k R}{(k R)^{2}}+\frac{\sin k R}{(k R)^{3}}\right] \\
3 A+B & =\frac{2 R}{\eta(r R)^{1 / 2}} \int_{0}^{\infty} d k \frac{1}{k^{2}} J_{1 / 2}(k r) J_{3 / 2}(k R)
\end{aligned}
$$


where $J_{\alpha}$ is the ordinary Bessel function. With the aid of Ref. 23, \#6.574, one obtains

$$
\begin{aligned}
3 A+B & =\frac{2}{3} R^{2} /(r \eta), & & r>R \\
& =(R / \eta)\left(1-r^{2} / 3 R^{2}\right), & & r<R
\end{aligned}
$$

In a similar way contraction with $\hat{\mathbf{r}} \hat{\mathbf{r}}$ leads to

$$
\begin{array}{rlrl}
A+B & =\frac{R^{2}}{2 \eta \pi^{2}} \int d \mathbf{k} \frac{\exp (i \mathbf{k} \cdot \mathbf{r})}{k^{2}}\left[1-(\hat{\mathbf{k}} \cdot \hat{\mathbf{r}})^{2}\right]\left[\frac{-\cos k R}{(k R)^{2}}+\frac{\sin k R}{(k R)^{3}}\right] \\
& =\left(1-R^{2} / 5 r^{2}\right) R^{2} /(3 \eta r), & r>R \\
& =\left(1-r^{2} / 5 R^{2}\right) R / 3 \eta, & & r<R
\end{array}
$$

Hence the velocity field has the form

$$
\begin{aligned}
\mathbf{u}(\mathbf{r})= & \frac{\hat{\mathbf{z}}}{\eta} \cdot\left(\left\{\left(\frac{R^{2}}{6 r}+\frac{R^{4}}{30 r^{3}}\right) W(r)+\left(\frac{R}{3}-\frac{2 r^{2}}{15 R}\right)[1-W(r)]\right\}\right. \\
& \left.+\left\{\left(\frac{R^{2}}{6 r}-\frac{R^{4}}{10 r^{3}}\right) W(r)+\frac{r^{2}}{15 R}[1-W(r)]\right\} \hat{\mathbf{r}} \hat{\mathbf{r}}\right)
\end{aligned}
$$

The density field follows as

$$
\begin{aligned}
\delta n(\mathbf{r})= & 4 \beta \pi R^{2} \frac{1}{(2 \pi)^{3}} \int d \mathbf{k} \frac{\exp (i \mathbf{k} \cdot \mathbf{r})}{i k}\left[\frac{\sin k R}{k R}+\frac{2 \cos k R}{(k R)^{2}}-\frac{2 \sin k R}{(k R)^{3}}\right](\hat{\mathbf{z}} \cdot \hat{\mathbf{k}}) \\
= & \frac{2 \beta R^{2}}{\pi r} \int_{0}^{\infty} d k\left(-\cos k r+\frac{\sin k r}{k r}\right) \\
& \times\left[\frac{\sin k R}{k R}+\frac{2 \cos k R}{(k R)^{2}}-\frac{2 \sin k R}{(k R)^{3}}\right](\hat{\mathbf{z}} \cdot \hat{\mathbf{r}}) \\
= & \beta R\left(\frac{R}{r}\right)^{1 / 2} \int_{0}^{\infty} d k J_{3 / 2}(k r)\left[-\frac{2}{3} J_{5 / 2}(k R)+\frac{1}{3} J_{1 / 2}(k R)\right](\hat{\mathbf{z}} \cdot \hat{\mathbf{r}}) \\
= & \frac{1}{3} \beta R^{2} / r^{2},(\hat{\mathbf{z}} \cdot \hat{\mathbf{r}}) \quad r>R \\
= & -\frac{2}{3} \beta r / R,(\hat{\mathbf{z}} \cdot \hat{\mathbf{r}}) \quad r<R
\end{aligned}
$$

All other calculations go in a completely analogous way. For example, a momentum source of type $\sigma_{p}=\hat{\mathbf{z}} \delta(r-R)$ becomes in Fourier language

$$
\sigma_{p}(\mathbf{k})=\int d \mathbf{r}[\exp (-i \mathbf{k} \cdot \mathbf{r})] \hat{\mathbf{z}} \delta(r-R)=4 \pi R^{2} \frac{\sin k R}{k R} \hat{\mathbf{z}}
$$


from which one obtains, with (D.7), for the source $\sigma_{2}=\hat{\mathbf{z}} \cdot(\mathbb{1}-\mathbf{r r}) \delta(r-R)$

$$
\begin{aligned}
\boldsymbol{\sigma}_{2}(\mathbf{k})= & 4 \pi R^{2} \hat{\mathbf{z}} \cdot\left\{\left[\frac{\sin k R}{k R}+\frac{\cos k R}{(k R)^{2}}-\frac{\sin k R}{(k R)^{3}}\right] \|\right. \\
& \left.-\left[\frac{\sin k R}{k R}+\frac{3 \cos k R}{(k R)^{2}}-\frac{3 \sin k R}{(k R)^{3}}\right] \hat{\mathbf{k}} \hat{\mathbf{k}}\right\}
\end{aligned}
$$

Similarly for the gradient-type momentum source

$$
\sigma_{4}^{\prime}=\nabla \cdot\left(\hat{\mathbf{r}} \hat{\mathbf{r}}-\frac{1}{3} \hat{1}\right)(\hat{\mathbf{r}} \cdot \hat{\mathbf{z}}) \delta(r-R)
$$

one obtains

$$
\begin{aligned}
\boldsymbol{\sigma}_{4}^{\prime}(\mathbf{k})= & \hat{\mathbf{z}} \cdot \int d \mathbf{r}[\exp (-i \mathbf{k} \cdot \mathbf{r})]\left[\hat{\mathbf{r}} \hat{\mathbf{r}}(i \mathbf{k} \cdot \mathbf{r})-\frac{1}{3} \hat{\mathbf{r}} i \mathbf{k}\right] \delta(r-R) \\
= & 4 \pi R \hat{\mathbf{z}} \cdot\left\{\left[\left(-\frac{\sin k R}{k R}-\frac{3 \cos k R}{(k R)^{2}}+\frac{3 \sin k R}{(k R)^{3}}\right) \mathbb{1}\right.\right. \\
& \left.+3\left(\frac{\sin k R}{k R}+\frac{3 \cos k R}{(k R)^{2}}-\frac{3 \sin k R}{(k R)^{3}}\right) \hat{\mathbf{k}} \hat{\mathbf{k}}\right] \\
& \left.-\frac{2}{3}\left(\cos k R-\frac{\sin k R}{(k R)^{2}}\right) \hat{\mathbf{k}} \hat{\mathbf{k}}\right\}
\end{aligned}
$$

By performing inverse Fourier transforms one can obtain again the fluid fields as function of position both outside and inside the sphere.

From the results obtained here one can also derive Eq. (7.9). On comparing (D.17) with (D.7) and (D.10), one finds that (7.9) would be satisfied if only the last term of the form

$$
(\hat{\mathbf{z}} \cdot \hat{\mathbf{k}}) \hat{\mathbf{k}}\left[\cos k R-\frac{\sin k R}{(k R)^{2}}\right]
$$

were not present on the right-hand side of (D.17). However, this term does not contribute to the velocity field, as follows from (D.2) and (D.3); and its contribution to the density field is of the form $(\hat{\mathbf{z}} \cdot \hat{\mathbf{r}}) \delta(r-R)$. (This is most easily seen by Fourier transforming the latter expression.) Looking back to (D.1), one easily understands the possibility for such a singular solution; with a velocity source $\nabla(\hat{\mathbf{z}} \cdot \hat{\mathbf{r}}) \delta(r-R)$, a density field $\beta(\hat{\mathbf{z}} \cdot \hat{\mathbf{r}}) \delta(r-R)$ plus a vanishing velocity field indeed solve the hydrodynamic equations. A similar $\delta$ function in the density field is generated by the density source $\sigma_{6}$, as follows from (D.5). In the density field generated by $S_{\mathrm{II}}^{c}$, given by (7.21) and (7.18b), both $\delta$-function contributions cancel each other.

Table I ists the hydrodynamic fields resulting from any given source term. The content of this table is entirely equivalent to (7.12)-(7.13). 


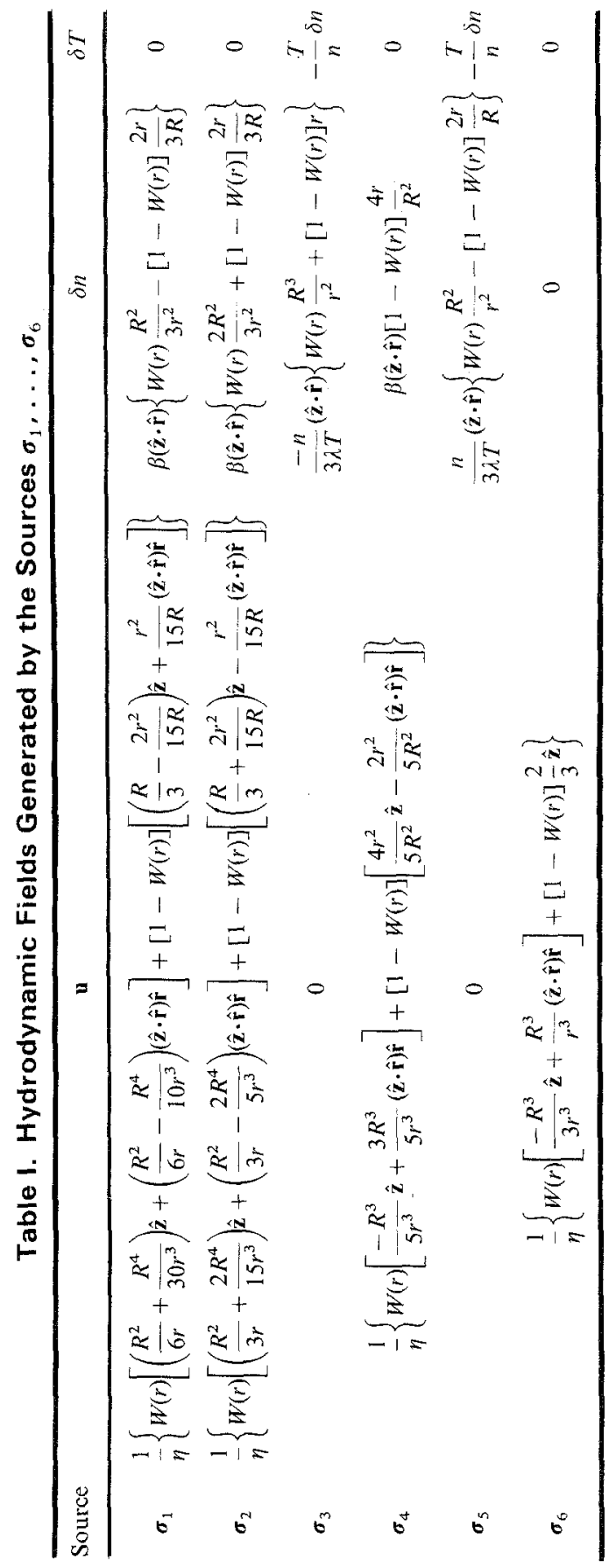




\section{ACKNOWLEDGMENTS}

The main part of the work reported in this paper was done during 1974-1976, while one of the authors ( HvB) held a postdoctoral position at the University of Maryland. The authors are especially indebted to Dr. C. F. McClure for his work on this problem in its early stages and for his continuing interest in it. The authors would also like to thank Profs. J. V. Sengers, R. Zwanzig, and E. G. D. Cohen and Dr. Yuen Han Kan for many helpful and stimulating conversations.

\section{REFERENCES}

1. J. R. Dorfman, H. van Beijeren, and C. F. McClure, Arch. Mech. Stosow. $28: 33$ (1976).

2. J. R. Dorfman and H. van Beijeren, in Statistical Mechanics, Part B, B. J. Berne, ed. (Plenum Press, New York, 1977).

3. S. Chapman and T. G. Cowling, The Mathematical Theory of Non-Uniform Gases, 3rd ed. (Cambridge University Press, London, 1970).

4. L. D. Landau and E. M. Lifshitz, Fluid Mechanics (Pergamon Press, New York, 1959), Chapter 2.

5. A. B. Bassett, Hydrodynamics (Dover, New York, 1961).

6. C. Cercignani, Theory and Application of the Boltzmann Equation (Elsevier, New York, 1975).

7. J. H. Ferziger and H. G. Kaper, Mathematical Theory of Transport Processes in Gases (North-Holland, Amsterdam, 1972; M. N. Kogan, Rarefied Gas Dynamics (Plenum Press, New York, 1969); see also papers by G. E. Uhlenbeck, H. Grad, and C. Cercignani in Eight International Symposium on Rarefied Gas Dynamics, K. Karamcheti, ed. (Academic Press, New York, 1974).

8. J. T. Hynes, Ann. Rev. Phys. Chem. 28:301 (1977).

9. E. H. Kennard, Kinetic Theory of Gases (McGraw-Hill, New York, 1938).

10. G. Scharf, Phys. Fluids 13:848 (1970).

11. J. A. McLennan and S. C. Chiu, Phys. Fluids 17:1146 (1974).

12. Y. P. Pao and D. R. Willis, Phys. Fluids 12:435 (1969).

13. Y. Kan, Ph.D. Dissertation, Department of Physics and Astronomy, University of Maryland, College Park (1975); Y. Kan, J. R. Dorfman, and J. V. Sengers, in Proceeding of the Seventh Symposium on Thermophysical Properties (American Society of Mechanical Engineers, New York, 1977).

14. C. Cercignani and C. D. Pagani, Phys. Fluids 11:1395 (1968); C. Cercignani, C. D. Pagani, and P. Bassanini, Phys. Fluids 11:1399 (1968); and C. Cercignani and C. D. Pagani, in Rarefield Gas Dynamics, Suppl. 4, C. L. Brunin, ed. (Academic Press, New York, 1967).

15. M. Van Dyke, Perturbation Methods in Fluid Mechanics (Parabolic Press, Stanford, 1975).

16. M. H. Ernst, J. R. Dorfman, W. R. Hoegy, and J. M. J. van Leeuwen, Physica 45:129 (1969).

17. D. Ronis, J. Kovac, and I. Oppenheim, Physica 88:215 (1977).

18. P. Résibois and M. DeLcener, Classical Kinetic Theory of Fluids (Wiley-Interscience, New York, 1977), Chapter V.

19. M. Klaus, Helv. Phys. Acta $48: 99$ (1974); 49:275 (1974); and H. van Beijeren, unpublished.

20. R. Zwanzig and M. Bixon, J. Fluid Mech. 69:21 (1975).

21. A. M. Albano, D. Bedeaux, and P. Mazur, Physica 80A:89 (1975).

22. P. M. Morse and H. Feshbach, Methods of Theoretical Physics (McGraw-Hill, New York, 1953).

23. I. S. Gradshteyn and J. M. Ryzhik, Table of Integrals, Series, and Products (Academic Press, New York, 1965). 